\title{
Protocol-Based Verification of Message-Passing Parallel Programs
}

\author{
Hugo A. López ${ }^{1}$ Eduardo R. B. Marques ${ }^{2} \quad$ Francisco Martins $^{2} \quad$ Nicholas $\mathrm{Ng}^{3}$ \\ César Santos $^{2} \quad$ Vasco Thudichum Vasconcelos $^{2} \quad$ Nobuko Yoshida $^{3}$ \\ ${ }^{1}$ Technical University of Denmark, Denmark $\quad{ }^{2}$ LaSIGE, Faculdade de Ciências, Universidade de Lisboa, Portugal \\ ${ }^{3}$ Imperial College London, London, UK
}

\begin{abstract}
We present ParTypes, a type-based methodology for the verification of Message Passing Interface (MPI) programs written in the $\mathrm{C}$ programming language. The aim is to statically verify programs against protocol specifications, enforcing properties such as fidelity and absence of deadlocks. We develop a protocol language based on a dependent type system for message-passing parallel programs, which includes various communication operators, such as point-to-point messages, broadcast, reduce, array scatter and gather. For the verification of a program against a given protocol, the protocol is first translated into a representation read by VCC, a software verifier for $\mathrm{C}$. We successfully verified several MPI programs in a running time that is independent of the number of processes or other input parameters. This contrasts with alternative techniques, notably model checking and runtime verification, that suffer from the state-explosion problem or that otherwise depend on parameters to the program itself. We experimentally evaluated our approach against state-ofthe-art tools for MPI to conclude that our approach offers a scalable solution.
\end{abstract}

Keywords Program verification, Parallel programming, MPI, Session types, Dependent types

\section{Introduction}

Background Message Passing Interface (MPI) [7] is the de facto standard for programming high performance parallel applications targeting hundreds of thousands of processing cores. MPI programs, written in C or Fortran, specify the

Permission to make digital or hard copies of all or part of this work for personal or classroom use is granted without fee provided that copies are not made or distributed for profit or commercial advantage and that copies bear this notice and the full citation on the first page. Copyrights for components of this work owned by others than the author(s) must be honored. Abstracting with credit is permitted. To copy otherwise, or republish, to post on servers or to redistribute to lists, requires prior specific permission and/or a fee. Request permissions from permissions@ acm.org.

OOPSLA '15, October 25-30, 2015, Pittsburgh/PA, USA.

Copyright is held by the owner/author(s). Publication rights licensed to ACM. ACM 978-1-nnnn-nnnn-n/yy/mm...\$15.00.

http://dx.doi.org/10.1145/nnnnnnn.nnnnnnnReprinted from OOPSLA '15, [Unknown

Proceedings], October 25-30, 2015, Pittsburgh/PA, USA, pp. 1-56. behavior of the various processes, each working on different data. Programs make calls to MPI primitives whenever they need to exchange data. MPI offers different forms of communication, notably, point-to-point and collective operators.

Developing MPI applications raises several problems: one can easily write code that causes processes to block indefinitely waiting for messages, or that exchange data of unexpected sorts or lengths. Verifying that such programs are exempt from communication errors is far from trivial. The state-of-the-art verification tools for MPI programs use advanced techniques such as runtime verification $[13,24,27,34]$ or symbolic execution and model checking $[10,13,24,29,32]$.

Runtime verification cannot guarantee the absence of faults. In addition, the task can become quite expensive due to the difficulty in producing meaningful tests, the time to run the whole test suite, and the need to run the test suite in hardware similar to that where the final application will eventually be deployed. On the other hand, model checking approaches frequently stumble upon the problem of scalability, since the search space grows exponentially with the number of processes. It is often the case that the verification of real applications limits the number of processes to less than a dozen [30].

Verification is further complicated by the different communication semantics for the various MPI primitives [29], or by the difficulty in disentangling processes' collective and individual control flow written on a single source file [1]. These also naturally arise in other more recent standards for message-based parallel programs, such as MCAPI [16].

We attack the problem of verifying C+MPI code using a type theory for parallel programs. In our framework a type describes a protocol, that is, the communication behavior of a program. Programs that conform to one such type are guaranteed to follow the protocol and not to run into deadlocks. The type system features a dependent type language including specific constructors for some of the most common communication primitives found in MPI, in addition to sequential composition, primitive recursion, and a form of 
collective choice. Our aim is to provide a typed verification basis for the safe development of parallel applications. The next paragraph explains our motivation via an example.

Motivation The finite differences algorithm illustrates the typical features present in a parallel application. Given an initial vector $X_{0}$, the algorithm calculates successive approximations to the solution $X_{1}, X_{2}, \ldots$, until a pre-defined maximum number of iterations has been reached. A distinguished process (usually process rank 0) disseminates the problem size via a broadcast operation. The same process then divides the input array among all processes. Each participant is responsible for computing its local part of the solution. Towards this end, in each iteration, each process exchanges boundary values with its left and right neighbours. When the pre-defined number of iterations is reached, process rank 0 obtains the global error via a reduce operation and collects the partial arrays in order to build a solution to the problem.

A stripped down version of the C+MPI code is depicted in Figure 1. Such code is extremely sensitive to variations in the use of MPI operations. For example, the omission of any send/receive operation (lines 11-24) leads to a deadlock where at least one process will be forever waiting for a complementary send or receive operation. Similarly, exchanging lines 21 and 22 leads to a deadlock where ranks $\theta$ and 1 will forever wait for one another. Other sorts of deadlocks may occur when different ranks perform different collective operations at the same time (say, rank 0 broadcasts and all other ranks reduce), or when one of the ranks decides to abandon, at an earlier stage, a loop comprising MPI primitives. It is also easy to use mismatching types or array lengths in MPI calls, thus compromising type and communication safety. Finally, it may not be obvious at all why one needs a three-branched conditional (lines 10-25) in order to perform the "simple" operation of sending a message to the left and then to the right, in a ring topology.

Solution We attack the problem from a programming language angle. In particular, we:

- Propose a protocol (type) language suited for describing the most common scenarios in the practice of parallel programming; and

- Statically check that programs conform to a given protocol, effectively guaranteeing the absence of deadlocks for well-typed programs, regardless of the number of process involved.

We develop our theory along the lines of intuitionistic type theory [18], demonstrating the soundness of our proposal via two main results: agreement of program reduction (cf., subject-reduction) and progress for programs.

Challenges Figure 2 presents a protocol for the finite differences algorithm. Special variable size represents the number of processes. The protocol captures the communica-

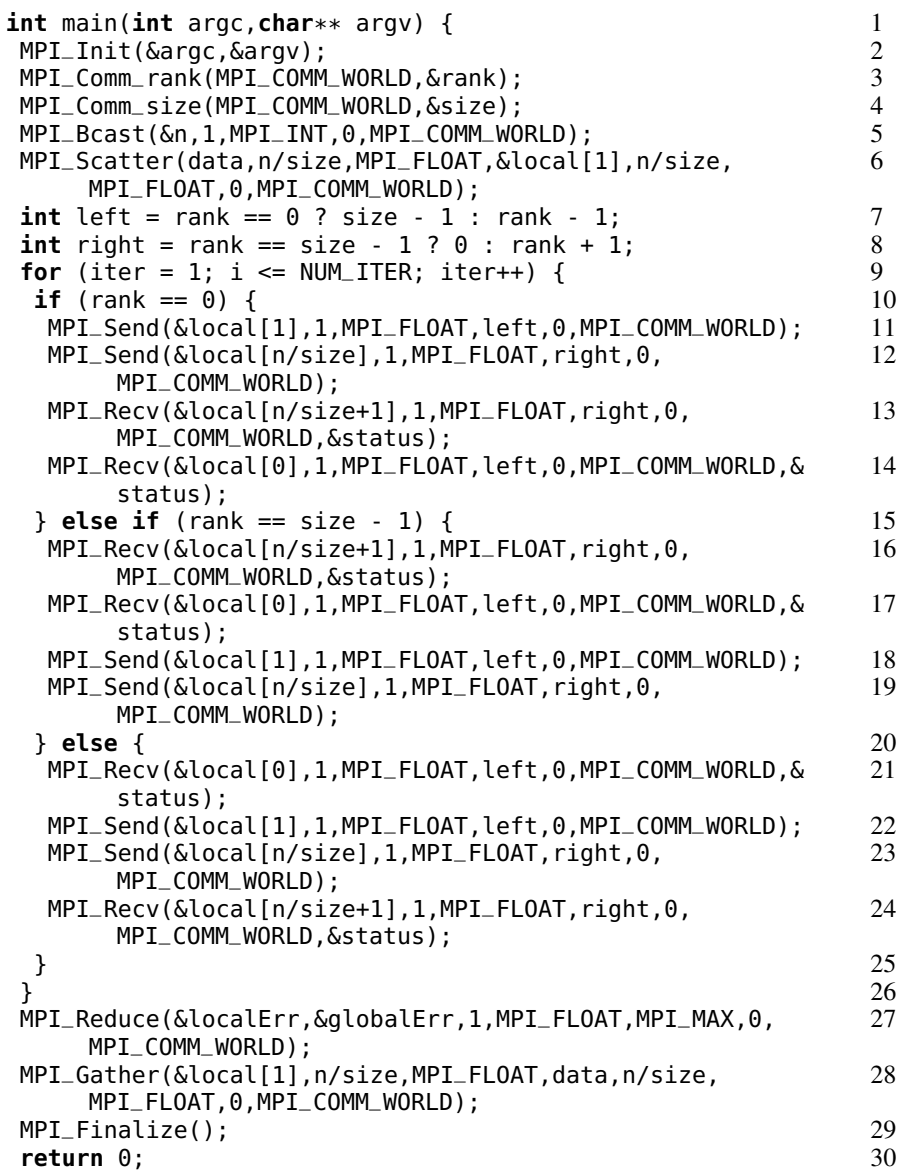

Figure 1. Excerpt of an MPI program for the finite differences problem (adapted from [8])

tion structure of the algorithm. A val constructor introduces the number of iterations; a broadcast operation initiated by rank 0 disseminates the problem dimension; scatter distributes the array. The external loop caters for the various iterations, whereas the inner one provides for the to-left and to-right message passing. The final reduce and gather collect the error and the solution.

There are two points worth noticing about this protocol. The first is that it talks about messages, whereas the source code mentions send and receive operations. The other is that its inner loop does not correspond to a loop in the program, instead it corresponds to a (three-branched) conditional. The protocol talks about the global behavior of the program, in a form inspired by session types [14]. The sole presence of a global protocol ensures deadlock freedom of programs that conform to the protocol.

Each individual process contributes to the global protocol. A novel type equivalence relation equates individual protocols against the global description, in such a way that, if each individual process conforms to its protocol, and all protocols are equivalent, then the program is deadlock free. 


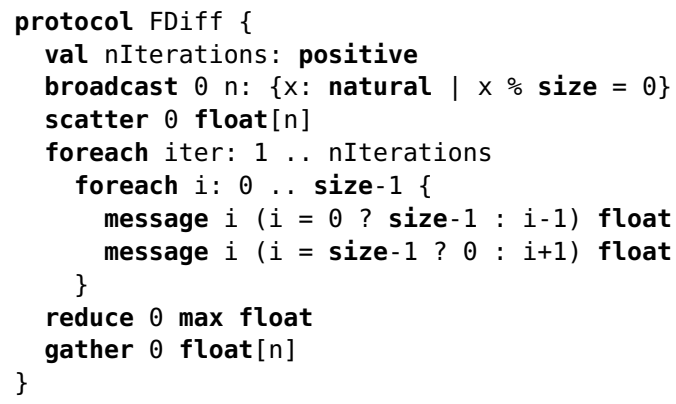

Figure 2. Protocol for the finite differences program

Type equivalence also naturally justifies the three branches in the source code, lines 10-25 in Figure 1.

Method In order to verify C+MPI source code against protocols we use the VCC deductive software verifier [2]. Our method can be summarised as follows.

- Write a protocol for the program (the protocol serves as further documentation for the program);

- Convert it to VCC;

- Introduce the required annotations in the $\mathrm{C}+\mathrm{MPI}$ source code; and

- Use VCC to check code conformance against the protocol.

If VCC runs successfully, then the program is guaranteed to follow the protocol and to be exempt from deadlocks, regardless of the number of processes, problem dimension, number of iterations, or any other variables.

\section{Contributions}

1. A dependently typed protocol language featuring primitive recursion and a separate static language of indices, along the lines of DML [36] and Omega [28];

2. A type checking system for a core parallel programming language ensuring a progress property for welltyped programs; and

3. A methodology for checking $\mathrm{C}+\mathrm{MPI}$ code against protocols, using a deductive program verifier.

Outline The next section presents a broad overview of the verification procedure. Sections 3 and 4 introduce the type theory, a core programming language, and the main results of the paper. Section 5 shows how we embodied the theory in VCC. Section 6 quantitatively compares our approach to state-of-the-art tools for the verification of C+MPI code. Section 7 discusses related work, and Section 8 concludes the paper.

\section{Overview of the verification procedure}

In order to check a program against a protocol, we need a program, a protocol, and a program verifier.
- Programs are written in the $\mathrm{C}$ programming language and make use of the MPI library interface;

- Protocols are written in a language described below;

- Programs are verified against a protocol using VCC.

In the sequel we detail how to construct protocols and how to prepare source code for VCC verification.

The protocol language by example We follow a step-bystep construction of the protocol for finite differences algorithm discussed in the introduction. The end result, we have seen, is in Figure 2.

In the beginning, process rank 0 broadcasts the problem size. We write this as

broadcast $\odot$ natural

That process rank 0 divides $X_{0}$ among all processes is rendered in ParTypes as a scatter operation.

scatter $\odot$ float []

Now, each process loops for a given number of iterations, nIterations. We write this as follows.

foreach i: 1..nIterations

nIterations is a variable that must be somehow introduced in the protocol. The variable denotes a value that must be known to all processes. Typically, there are two ways for processes to get to know this value:

- The value is exchanged resorting to a collective communications operation, in such a way that all processes get to know it, or

- The value is known to all processes before computation starts, for example because it is hardwired in the source code or is read from the command line.

For the former case we could for instance add another broadcast operation in the first lines of the protocol. For the latter, the protocol language relies on the val constructor, allowing one to introduce a program value in the type:

val nIterations: positive

Either solution would solve the problem. If a broadcast is used then processes must engage in a broadcast operation; if val is chosen then no value exchange is needed, but the programmer must identify the value in the source code that will inhabit nIterations.

We may now continue analyzing the loop body. In each iteration, each process sends a message to its left neighbor and another message to its right neighbor. Such an operation is again described as a foreach construct that iterates over all processes. The first process is 0 ; the last is size-1, where size is a distinguished variable that represents the number of processes. The inner loop is then written as follows.

foreach $i: 0 \ldots$ size-1 
When $i$ is the rank of a process, an expression of the form i=size- 1 ? $\odot$ : i+1 denotes its right neighbor. Similarly, the left neighbor is $i=0$ ? size -1 : $i-1$.

To send a message containing a value of a datatype $D$, from process rank $r 1$ to rank $r 2$ we write message $r 1 \quad r 2 D$. In this way, to send a message containing a floating point number to the left process, followed by a message to the right process, we write.

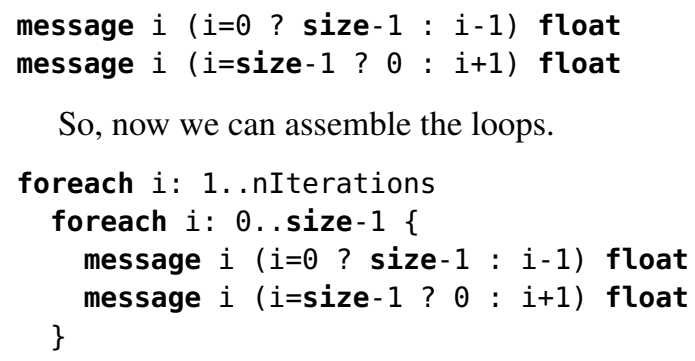

So, now we can assemble the loops.

Once the loop is completed, process rank 0 obtains the global error. Towards this end, each process proposes a floating point number representing the local error. Rank 0 then reads the maximum of all these values. We write all this as follows.

\section{reduce $\theta$ max float}

Finally, process rank 0 collects the partial arrays and builds a solution $X_{n}$ to the problem. This calls for a gather operation.

\section{gather $\theta$ float []}

Before we put all the operations together in a protocol, we need to discuss the nature of the arrays distributed and collected in the scatter and gather operations. Scatter distributes $X_{0}$, dividing it in small pieces; gather collects the subarrays to build $X_{n}$. The arrays in scatter/gather protocols always refer to the whole array, not to the subarrays. So, we instead write:

\section{scatter $\odot$ float $[n]$ \\ $\ldots$ \\ gather 0 float $[n]$}

Variable $n$ must be introduced somehow (by means of a val, broadcast, or allreduce). In this case $n$ is exactly the problem size that was broadcast before. So we name the value that rank $\theta$ provides as follows.

\section{broadcast $\odot \mathrm{n}$ : natural}

But $\mathrm{n}$ cannot be an arbitrary non-negative number. It must evenly divide $X_{0}$. In this way, each process gets a part of $X_{0}$ of equal length, namely length $(X \theta) /$ size, and we do not risk accessing out-of-bound positions when manipulating the subarrays. So we would like to make sure that the length of $X_{0}$ is such that length $(X \theta) \%$ size $=0$. For this we use a refinement datatype. Rather that saying that $n$ is a natural number we say that it is of datatype $\{x$ : natural $\mid x \%$ size $=0\}$.
As an aside, datatype natural can be expressed as $\{x$ : integer $\mid x>=0\}$. Similarly, datatype positive abbreviates $\{x$ :integer $\mid x>0\}$. Finally, syntax float $[n]$ is the abbreviation of a refinement type $\{x$ : float [] $\mid$ length $(x)=n\}$.

The topology underlying the protocol for the finite differences (Figure 2) is that of a ring: a linear array with a wraparound link. If a different mapping of ranks to processes is to be used, a new protocol must be derived. It turns out that the language of protocols is flexible enough to encode topologies in integer arrays. Such a topology may then be made known to all processes, in such a way that processes exchange messages as per the particular topology. This flexibility is particularly useful for applications that dynamically adequate the protocol to, say, the load of messages exchanged. A datatype of the form

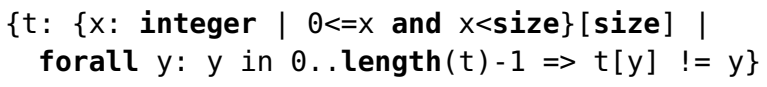

encodes a one-dimensional network topology, where $t[x]=y$ means $\mathrm{x}$ is a direct neighbor of $\mathrm{y}$ : each node has one direct neighbor (a number between 0 and size-1) that is different from itself. Such a type, call it D, can be distributed among all processes by, say, rank $\odot$.

broadcast $\odot$ topology:D

Thereafter each process can exchange a message with its neighbor, as in:

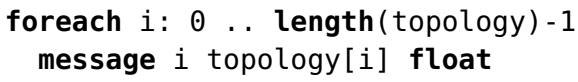

A right-to-left ring topology of length five can be encoded as $[4,0,1,2,3]$.

How can one encode a topology where not all processes have direct neighbors, such as a star or a line? One possibility is to weaken the above condition on the elements of the array, while strengthening the subsequent message passing loop. We could for example drop the restriction that $\mathrm{t}[\mathrm{y}] \mathrm{l}=\mathrm{y}$ and encode a right-to-left line of length five as $[0,0,1,2,3]$, a 0 -centered star as $[0,0,0,0,0]$, and a full binary 0-rooted tree of depth 3 as $[0,0,0,1,1,2,2]$. In all cases, rank 0 has no direct neighbor. And this causes a problem if we try to send a message from $i$ to topology [i], as in the above example.

Given that the topology is a data structure known to all processes we can make use of a new primitive called collective choice. We start by broadcasting the topology and enter the loop as before. Then, within the loop, a message is exchanged only if the topology array contains a valid entry.

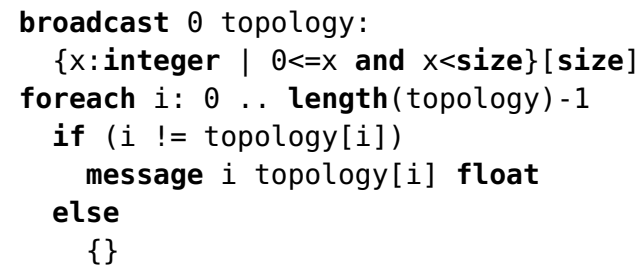


Arbitrary topologies can be encoded, e.g., using an adjacency matrix. More examples of protocols can be found in Section 6.

Verifying $C+M P I$ code against a protocol In order to verify a program against a protocol, we need:

- The C+MPI source code;

- The protocol in VCC syntax (a C header file);

- The type theory in VCC format (another header file).

The protocol in VCC syntax can be generated by the ParTypes Eclipse plugin or by a web service [23]. Both check, in addition, the good formation of the protocol. The ParTypes VCC library can be obtained from the project's web site [23].

All that remains is preparing the source code, so that it may be successfully verified by VCC. First, VCC limitations force us to adapt the $\mathrm{C}$ source code. In particular, $\mathrm{VCC}$ does not support a theory for floating point numbers, and functions with a variable number of arguments. Thus, we must filter out lines that contain, e.g., printf or scanf, and also adapt floating point code that may have impact on the verification process, e.g., control flow predicates involving expressions of type float or double. In this process, we must preserve the control structure of the program, including calls to MPI primitives and variable declarations. The resulting program must still compile and exchange the messages the original program was intended to.

Next, our approach requires introducing some annotations, including:

- Those that distinguish $\mathrm{C}$ loops to be matched against foreach protocols. The core language described in Section 4 uses for expressions for code that is supposed to match a foreach protocol and while loops for all other purposes; $\mathrm{C}$ does not make this distinction;

- Those that distinguish $\mathrm{C}$ conditionals to be matched against collective choice protocols, if -else. Again, our core language distinguishes ifc expressions to be matched against collective choices from if expressions used for all other purposes, while $\mathrm{C}$ does not;

- The $\mathrm{C}$ expression that matches a val type; and

- Annotations that guide VCC in matching a foreach-type against a non-loop $\mathrm{C}$ constructor.

For $\mathrm{C}$ functions that make use of MPI operations we have two options:

- Inline the code (MPI programs are usually non recursive), or

- Write a contract to the function in the form of a pre- and a post-condition, stating the entry and exit values of $p$, the ghost variable that holds the protocol.

Further details on the annotations required by ParTypes are provided in Section 5.
We are finally in a position to run VCC on the source code. If VCC reports no errors, the program complies with the protocol, and we may conclude that it faithfully follows the protocol and is, in particular, free from deadlocks.

\section{The type theory}

This section introduces the notion of type and the novel notion of type equivalence. Type equivalence is shown to be decidable.

Index terms Types rely on two base sets: that of variables (denoted $x, y, z)$, and that of integer values $(k, l, m, n)$. There are two distinguished variables: size and rank; we use them to denote the total number of processes and the unique number of a given process, respectively. It will always be the case that $1 \leq$ rank $\leq$ size. Unlike the case of MPI, the ranks in our type theory run from 1 . We start by discussing a few notions types rely on.

Index terms, $i$, describe the values types may depend upon. Our language counts with variables, integer, and arithmetic operations, as well as the usual array operations: creation $\left[i_{1}, \ldots, i_{n}\right]$, access $i_{1}\left[i_{2}\right]$, and length len $(i)$. Index term formation further includes the standard refinement introduction rule and datatype subsumption [11].

Datatypes Datatypes, $D$, describe integer values (int), arrays of an arbitrary datatype ( $D$ array), and refinements of the form $\{x: D \mid p\}$. Refinement datatypes allow one to describe, e.g., integer values smaller than a given index term $i$, such as $\{y:$ int $\mid y \leq i\}$, or arrays of a given length $n$, as in $\{a$ : float array $\mid \operatorname{len}(a)=n\}$. Datatypes rely on propositions over index terms, including relational operations and conjunction.

All formation rules depend on contexts, intuitively ordered maps from variables into datatypes. Contexts are also subject to formation rules. Symbol $\varepsilon$ denotes the empty context. A notion of subtyping is defined for datatypes, $\Gamma \vdash$ $D_{1}<: D_{2}$. The rules are standard and include those for refinement datatypes [11]. They allow us to conclude that $\varepsilon \vdash\{a$ : float array $\mid$ len $(a)=512\}<$ : float array.

Type formation We are now in a position to discuss types and type formation. The rules for type formation are in Figure 3. Given $\Gamma$ and $T$, if one can deduce $\Gamma \vdash T$ : type, then $T$ is a (well-formed) type under a (well-formed) context $\Gamma$. We do not provide a grammar for the constructs of our language; such a grammar, if desired, can be recovered from the blue text in the relevant figures.

A type of the form message $i_{1} i_{2} D$ describes a point-topoint communication, from the $i_{1}$-ranked process to the $i_{2}$ ranked process, of a value of datatype $D$. Both index terms must denote valid ranks, that is, they must lie between 1 , the first rank, and size, the number of processes. Furthermore, the sending and the receiving processes must be different from each other, since under our semantics, a message from, say, rank 2 to rank 2 leads to a deadlock (see Section 4). 


$$
\begin{aligned}
& \frac{\Gamma \vdash 1 \leq i_{1}, i_{2} \leq \text { size } \wedge i_{1} \neq i_{2} \text { true } \quad \Gamma \vdash D: \text { dtype }}{\Gamma \vdash \text { message } i_{1} i_{2} D: \text { type }} \\
& \frac{\Gamma \vdash 1 \leq i \leq \text { size true }}{\Gamma \vdash \text { reduce } i \text { : type }} \\
& \frac{\Gamma \vdash 1 \leq i \leq \operatorname{size} \quad \Gamma \vdash D<:\left\{x: D^{\prime} \text { array } \mid \operatorname{len}(x) \% \text { size }=0\right\}}{\Gamma \vdash \operatorname{scatter} i D: \text { type }} \\
& \frac{\Gamma \vdash 1 \leq i \leq \operatorname{size} \quad \Gamma \vdash D<:\left\{x: D^{\prime} \text { array } \mid \operatorname{len}(x) \% \text { size }=0\right\}}{\Gamma \vdash \text { gather } i D: \text { type }} \\
& \frac{\Gamma \vdash 1 \leq i \leq \text { size true } \quad \Gamma, x: D \vdash T: \text { type }}{\Gamma \vdash \text { broadcast } i x: D . T: \text { type }} \\
& \frac{\Gamma, x: D \vdash T: \text { type }}{\Gamma \vdash \operatorname{val} x: D . T: \text { type }} \frac{\Gamma, x:\{y: \text { int } \mid y \leq i\} \vdash T: \text { type }}{\Gamma \vdash \forall x \leq i . T: \text { type }} \\
& \frac{\Gamma \vdash p: \text { prop } \Gamma \vdash T_{1}: \text { type } \Gamma \vdash T_{2}: \text { type }}{\Gamma \vdash p ? T_{1}: T_{2}: \text { type }} \\
& \frac{\Gamma \vdash T_{1}: \text { type } \Gamma \vdash T_{2}: \text { type }}{\Gamma \vdash T_{1} ; T_{2}: \text { type }} \frac{\Gamma: \text { context }}{\Gamma \vdash \text { skip : type }}
\end{aligned}
$$

Figure 3. Type formation rules

A type of the form reduce $i$ denotes a collective operation whereby all processes contribute with values that are used to produce a result (say, the maximum). This value is then transmitted to the $i$-ranked process, usually known as the root process.

A type of the form scatter $i D$ describes a collective operation by which the $i$-ranked process (the root process) distributes an array among all processes, including itself. The type formation rule requires $i$ to be a valid rank, and $D$ to be an array. Type gather $i D$ denotes the inverse operation, whereby each process proposes an array of identical length, the concatenation of which is delivered to the root process. In both operations, $D$ describes the whole array, that is the array that is distributed in scatter or assembled in gather.

A type of the form broadcast $i x: D . T$ denotes a collective communication whereby the root process transmits a value of type $D$ to all processes (including itself). The continuation type $T$ may refer to the value transmitted via variable $x$. Type val $x: D . T$ is the dependent product type. In our case, it denotes a collective operation whereby all processes agree on a common value of datatype $D$, without resorting to communication. Typical applications include program constants and command-line values that programs may depend upon. A type $p ? T_{1}: T_{2}$ denotes a collective conditional, whereby all processes jointly decide on proceeding as $T_{1}$ or as $T_{2}$, again without resorting to communication. The type assignment system in Section 4 makes sure that the value of $p$ is common to all processes.

Type $T_{1} ; T_{2}$ describes a computation that first performs the operations as described by $T_{1}$ and then those described by $T_{2}$. Type skip describes any computation that does not engage in communication. skip-typed processes are not neces-

$$
\begin{gathered}
\frac{(\Gamma \vdash T: \text { type })}{\Gamma \vdash T ; \text { skip } \equiv T} \quad \frac{(\Gamma \vdash T: \text { type })}{\Gamma \vdash \text { skip } T \equiv T} \\
\frac{\left(\Gamma \vdash T_{1}, T_{2}, T_{3}: \text { type }\right)}{\Gamma \vdash\left(T_{1} ; T_{2}\right) ; T_{3} \equiv T_{1} ;\left(T_{2} ; T_{3}\right)} \\
\frac{\Gamma \vdash i<1 \text { true } \quad(\Gamma, x:\{y: \text { int } \mid y \leq i\} \vdash T: \text { type })}{\Gamma \vdash \forall x \leq i . T \equiv \text { skip }} \\
\frac{\Gamma \vdash i \geq 1 \text { true } \quad(\Gamma, x:\{y: \text { int } \mid y \leq i\} \vdash T: \text { type })}{\Gamma \vdash \forall x \leq i . T \equiv(T\{i / x\} ; \forall x \leq i-1 . T)} \\
\Gamma \vdash i_{1}, i_{2} \neq \text { rank true } \\
\left(\Gamma \vdash 1 \leq i_{1}, i_{2} \leq \text { size } \wedge i_{1} \neq i_{2} \text { true }\right) \quad(\Gamma \vdash D: \text { dtype })
\end{gathered}
$$

Figure 4. Type equality (excerpt)

sarily halted; they may still perform local operations. Finally, type of the form $\forall x \leq i . T$ is a concrete instance of primitive recursion. A type $\forall x \leq i . T$ uniquely determines an indexed family of types $T\{i / x\} ; T\{i-1 / x\} ; \ldots ; T\{1 / x\}$; skip. ${ }^{1}$

In addition to the above type constructors, others could be easily added, either as primitives or as derived constructors, including: software barriers (adapted, e.g., from reduce), allreduce (defined as reduce followed by broadcast), and allgather (gather followed by broadcast).

The reader may have noticed that types such as message or reduce do not introduce value dependencies, whereas others such as broadcast and val do. The continuation of a message type, if exists, is captured by sequential composition, as in message 12 float; message 21 int. The continuation of a broadcast is built into the type constructor itself; as in broadcast $1 x$ : int. broadcast $1 y:\{z:$ int $\mid z \geq x\}$.skip. The fundamental reason for the difference lies in the target of the values exchanged. In the cases of message and reduce values are transmitted to a unique process, namely the root process. In the case of broadcast all processes receive the same value. This value may then be safely substituted in the continuation of the types for all processes, thus preserving the good properties of types.

Type formation is decidable; we have written an Eclipse plugin, using an SMT solver, that checks protocol formation [23].

Term type equality Type equality plays a central role in dependent type systems. In our case, type equality includes the monoidal rules for semicolon and skip, the (base and step) rules for primitive recursion, and a form of projection of message types. The rules in Figure 4 determine what it means for two types to be equal under a given context (rules pertaining to congruence and equivalence omitted).

Premises enclosed in parenthesis ensure type formation, playing no other role in the definition of type equality. If we

\footnotetext{
${ }^{1}$ Contrary to the concrete syntax for protocols (cf. Figure 2), primitive
} 


$$
\begin{aligned}
& \frac{\Gamma \vdash i_{1}, i_{2} \neq \text { rank true }\left(\Gamma \vdash \text { message } i_{1} i_{2} D: \text { type }\right)}{\Gamma \vdash \text { message } i_{1} i_{2} D \Rightarrow \text { skip }} \\
& \frac{\Gamma \vdash i<1 \text { true }(\Gamma \vdash \forall x \leq i . T: \text { type })}{\Gamma \vdash \forall x \leq i . T \Rightarrow \text { skip }} \\
& \Gamma \vdash i \geq 1 \text { true } \quad \Gamma \vdash T\{i / x\} \Rightarrow T^{\prime} \\
& \frac{\Gamma \vdash \forall x \leq i-1 . T \Rightarrow T^{\prime \prime} \quad(\Gamma \vdash \forall x \leq i . T: \text { type })}{\Gamma \vdash \forall x \leq i . T \Rightarrow T^{\prime} ; T^{\prime \prime}}
\end{aligned}
$$

Figure 5. Type conversion (excerpt)

abbreviate a context entry of the form size: $\{x:$ int $\mid x=3\}$ by size $=3$, we can easily show that:

$$
\begin{aligned}
\text { size }=3 \vdash \forall j \leq \text { size.message } j(j \% \text { size }+1) \equiv \\
\text { message } 31 \text {; message } 23 ; \text { message } 12
\end{aligned}
$$

The last rule in Figure 4 says that a message type that plays no role for a given process rank is equal to skip. The rule effectively allows one to project a given type onto a given rank, a notion introduced in the context of multi-party session types [14], here cleanly captured as type equality. When projecting the above type onto rank 2 we obtain the following type equality,

$$
\begin{array}{r}
\text { size }=3, \text { rank }=2 \vdash \forall j \leq \text { size message } j(j \% \text { size }+1) \equiv \\
\text { message } 23 ; \text { message } 12
\end{array}
$$

in such a way that the $\forall$-type is effectively equivalent to the sequential composition of two messages, when judged under rank 2.

Decidability of type equivalence The proof relies on a type conversion relation, and follows the strategy of Coquand [3], albeit in a simplified form. The type conversion relation expands $\forall$-types and projects message types. The result is a type where $\forall$ cannot be further expanded and each message is not equivalent to skip. The relevant rules are in Figure 5; the remaining are the ten congruence rules. The following lemmas establish validity and confluence for the type conversion relation.

Lemma 3.1 (agreement for type conversion). If $\Gamma \vdash T \Rightarrow$ $T^{\prime}$ then $\Gamma \vdash T$ : type and $\Gamma \vdash T^{\prime}$ : type.

Lemma 3.2 (type conversion is deterministic). If $\Gamma \vdash T \Rightarrow$ $T^{\prime}$ and $\Gamma \vdash T \Rightarrow T^{\prime \prime}$, then $T^{\prime}=T^{\prime \prime}$.

The type equivalence algorithm relies on a further relation, structural congruence $\equiv_{c}$, defined as the smallest congruence relation that incorporates the commutative monoidal rules for semi-colon and skip.

It should be easy to see that conversion is (strongly) normalizing (primitive recursion is and projection reduces the size of terms). Structural congruence is also decidable (by converting types to skip-terminated lists and checking for equality). The algorithmic type equality, $\equiv_{a}$, is defined by the following rule,

$$
\frac{\Gamma \vdash T_{1} \Rightarrow T_{1}^{\prime} \quad \Gamma \vdash T_{2} \Rightarrow T_{2}^{\prime} \quad \Gamma \vdash T_{1}^{\prime} \equiv_{c} T_{2}^{\prime}}{\Gamma \vdash T_{1} \equiv_{a} T_{2}}
$$

and works as follows: given a context $\Gamma$ and two types $T_{1}$ and $T_{2}$, apply type conversion to both, obtaining $T_{1}^{\prime}$ and $T_{2}^{\prime}$. Then check these types for congruence.

We can easily show that algorithmic type equality is sound and complete with respect to type equivalence.

Theorem 3.3 (correctness of algorithmic type equality). $\Gamma \vdash$ $T_{1} \equiv_{a} T_{2}$ if and only if $\Gamma \vdash T_{1} \equiv T_{2}$.

Program types Program types are vectors of term types. Not all vectors are nevertheless of interest. Program types in particular must not deadlock. Below are a few candidates that, albeit composed of term types, cannot be judged as program types. For the sake of brevity we once more omit the datatype in types.

$$
\begin{gathered}
\text { (message } 12),(\text { message } 21) \\
(\text { scatter } 1),(\text { reduce } 1)
\end{gathered}
$$

(msg 13 ; scatter 1$),($ msg $13 ;$ reduce 1$),(\operatorname{msg} 13 ;$ scatter 1$)$

(msg 31 ; msg 12 ), (msg 12 ; msg 23 ), (msg 23 ; msg 31 )

The first vector of types is blocked since process rank 1 intends to send a message to rank 2 , whereas rank 2 is ready to send a message to rank 1 . In the second vector, rank 1 is trying to distribute an array, whereas rank 2 is not ready to receive its part. The third case involves a 1-3 message that leads to a deadlocked situation, namely (scatter 1$)$, (message 13 ; reduce 1$)$, (scatter 1 ); notice that the second type is equivalent to reduce 1 . The fourth case involves a circular waiting situation: the message between 3 and 1 cannot happen before that of 2 and 3 (see type for rank 3); the 2-3 message cannot happen before the 1-2 (type for rank 2); and finally, the 1-2 message cannot happen before the 3-1 message (type for rank 1). We judge such vector of types as not constituting program types.

We abbreviate context size $=n$, rank $=k$ to $\Gamma^{n, k}$. The rule defining what constitutes a program type, that is determining the meaning of assertions $S$ : ptype, is defined as follows.

$$
\frac{\Gamma^{n, k} \vdash T_{k} \equiv T: \text { type } \quad(1 \leq k \leq n)}{T_{1}, \ldots, T_{n}: \text { ptype }}
$$

The central intuition of a program type is that it describes a non-deadlocked computation, that is, a computation that is either halted or that may reduce. With this in mind it is easy to understand that all types must be aligned (if one

recursion decreases the loop variable. 


$$
\begin{aligned}
& \frac{\Gamma \vdash 1 \leq i_{1} \leq \text { size } \wedge i_{1} \neq \text { rank true } \quad \Gamma \vdash i_{2}: D}{\Gamma \vdash \text { send } i_{1} i_{2}: \text { message rank } i_{1} D} \\
& \frac{\Gamma \vdash 1 \leq i_{1} \leq \text { size } \wedge i_{1} \neq \text { rank true } \quad \Gamma \vdash i_{2}: D \text { ref }}{\Gamma \vdash \text { receive } i_{1} i_{2}: \text { message } i_{1} \text { rank } D} \\
& \Gamma \vdash 1 \leq i_{1} \leq \text { size true } \\
& \Gamma \vdash i_{2}: D \quad \Gamma, x: D \vdash e: T \quad \text { rank } \notin \mathrm{fv}\left(i_{1}\right) \\
& \Gamma \vdash \text { let } x: D=\text { broadcast } i_{1} i_{2} \text { in } e: \text { broadcast } i_{1} x: D . T \\
& \Gamma \vdash 1 \leq i_{1} \leq \text { size true } \\
& \Gamma \vdash i_{2} \text { : float array ref } \quad \Gamma \text {, rank }=i_{1} \vdash i_{3}: \text { float }\left[\operatorname{size} * \operatorname{len}\left(i_{2}\right)\right] \\
& \Gamma \vdash \text { scatter } i_{1} i_{2} i_{3}: \text { scatter } i_{1} \text { float [size } * \operatorname{len}\left(i_{2}\right) \text { ] } \\
& \Gamma \vdash 1 \leq i_{1} \leq \text { size true } \Gamma \vdash i_{2} \text { : float array } \\
& \Gamma \text {, rank }=i_{1} \vdash i_{3}: \text { float }\left[\operatorname{size} * \text { len }\left(i_{2}\right)\right] \text { ref } \\
& \overline{\Gamma \vdash \text { gather } i_{1} i_{2} i_{3}: \text { gather } i_{1} \text { float }\left[\operatorname{size} * \operatorname{len}\left(i_{2}\right)\right]} \\
& \frac{\Gamma \vdash e_{1}: T_{1} \quad \Gamma \vdash e_{2}: T_{2}}{\Gamma \vdash e_{1} ; e_{2}: T_{1} ; T_{2}} \quad \frac{\Gamma, x:\{y: \text { int } \mid y \leq i\} \vdash e: T}{\Gamma \vdash \text { for } x: i . .1 \text { do } e: \forall x \leq i . T} \\
& \frac{\Gamma,\{p\} \vdash e: \text { skip }}{\Gamma \vdash \text { while } p \text { do } e \text { : skip }} \quad \frac{\Gamma \vdash e: T_{1} \quad \Gamma \vdash T_{1} \equiv T_{2}}{\Gamma \vdash e: T_{2}}
\end{aligned}
$$

In all rules, $T$ and $D$ contain no ref datatypes.

Figure 6. Expression formation (excerpt)

is reduce $i$, then all are reduce $j$ and $\Gamma \vdash i=j$ true). One exception is the non-collective message types. Yet, even in this case we require type equality by taking advantage of the "projection" rule in type equality, so that, for example, message 13 , skip, message 13 is a program type.

\section{A core message-passing programming language}

This section introduces a core Multiple-Program-MultipleData message-passing imperative programming language and its main results: agreement (cf. subject reduction) and progress for programs.

References To deal with imperative features, we introduce the notion of references. We rely on an extra base set, that of reference identifiers, ranged over by $r$. A new datatype, $D$ ref, describes references to values of type $D$. Four new index terms are introduced: references $r$, and the conventional operations on references: creation mkref $i$, dereference $! i$, and assignment $i_{1}:=i_{2}$.

We designed our programming language in such a way that it directly handles the index terms present in types. The pure index terms introduced in Section 3 are however extended with side effects, such as reference creation and assignment. The meaning of expressions with effects when they occur as index objects to type families is undetermined. For this reason we are careful in requiring index objects appearing in types to remain pure.

Expressions The constructors of our language can intuitively be divided in two parts: conventional expressions usu- ally found in a while-language and communication-specific expressions. A selection of the expression formation rules is in Figure 6; the remaining (skip, val, reduce, collective conditional ifc, conventional conditional if, and let) are standard.

In an expression of the form send $i_{1} i_{2}$, index term $i_{1}$ (of datatype int) denotes the target process and index term $i_{2}$ (of datatype $D$ ) describes the value to be sent. The type of the send expression is message rank $i_{1} D$, representing a message from process rank to process $i_{1}$ containing a value of datatype $D$. The premises come naturally if one considers the hypothesis necessary for message rank $i_{1} D$ to be considered a type under context $\Gamma$, namely, $i_{1}$ must denote a valid process number and must be different from the sender's rank. The value to be sent, $i_{2}$, must be of datatype $D$, so that it conforms to the value the message is supposed to exchange.

An expression of the form receive $i_{1} i_{2}$ denotes the reception of a value (of datatype $D$ ) from process $i_{1}$. The value is stored in the reference (of datatype $D$ ref) denoted by index term $i_{2}$. The type of the expression is message $i_{1}$ rank $D$, expressing the fact that a message is transmitted from process $i_{1}$ to the target process rank.

In a broadcast expression, index term $i_{1}$ denotes the root process and index term $i_{2}$ the value to be distributed. The root process cannot refer to the special variable rank, for this has different values at different processes, precluding all processes from agreeing on a common root process. Contrary to the expressions studied so far, where the object of communications is stored in a reference, the value distributed by the root process is collected in a variable $x$ and made available to an explicit continuation expression $e$. This strategy provides for datatype dependency in broadcast operations, while keeping the expression and the type aligned, as made clear by the type formation rule: variable $x$ (of datatype $D$ ) is moved into the context to type the continuation, while retaining its presence in the dependent type for broadcast.

The scatter expression requires three index term arguments: the first is the process that distributes the array (the root), the second is the reference that will hold the subarray, and the third is the array to be distributed. The premises reflect these conditions; notice how the types for the arrays embody the relation between their lengths. As discussed in Section 2, notation $D[p]$ abbreviates the refinement datatype of the form $\{a: D$ array $\mid \operatorname{len}(a)=p\}$. The rule for the gather expression is similar, except that the order of the last two parameters is reversed: $i_{2}$ denotes the subarrays proposed by each process and $i_{3}$ the array to be assembled at the root.

In both expressions, ref datatypes denote values written at each process (as in receive), and the last index denotes an expression that is evaluated only at the root process. We omit similar rules for arrays on integer values, as well as multidimensional arrays.

The expression formation rule for sequential composition $e_{1} ; e_{2}$ is particular: its type, $T_{1} ; T_{2}$, is composed of the types $T_{1}$ and $T_{2}$ for expressions $e_{1}$ and $e_{2}$. The conventional rule 


$$
\begin{gathered}
\frac{(\rho, i) \downarrow^{n, k}\left(\rho^{\prime}, v\right): D \quad\left(\Gamma^{n, k}, \rho, x: D \vdash e: T\right) \quad(x \notin \mathrm{fv}(T))}{(\rho, \text { let } x: D=i \text { in } e) \rightarrow^{n, k}\left(\rho^{\prime}, e\{v / x\}\right)} \\
\frac{\left(\Gamma^{n, k}, p, \rho \vdash e_{1}: T\right) \quad\left(\Gamma^{n, k}, \neg p, \rho \vdash e_{2}: T\right)}{\left(\rho, \text { if } p \text { then } e_{1} \text { else } e_{2}\right) \rightarrow^{n, k}\left(\rho, e_{1}\right)}
\end{gathered}
$$

Figure 7. Process reduction (a flavor)

is obtained when $e_{1}$ does not engage in communication operations, in which case its type is skip, and we know that $\Gamma \vdash$ skip; $T_{2} \equiv T_{2}$.

In expression for $x: i . .1$ do $e$, variable $x$ takes values $i, i-$ $1, \ldots, 1$ in each different iteration of the loop. The rule for while requires $e$ to be of type skip, not allowing the loop to perform any communication action. An entry of the form $\{p\}$ in a context abbreviates $x:\{x:$ int $\mid p\}$, for $x$ a fresh variable [11]. Non skip-types in the body of while loops may lead to deadlocks, since processes are not guaranteed to run the same number of iterations. If communications are required in a loop body, then a for loop must be used. Finally, the last rule in our selection introduces type equality in expression formation.

Stores For the operational semantics we make use of stores, maps from reference identifiers into values. Stores can be easily converted into contexts. A store entry of the form $r:=v$ is transformed into a context entry $r: D$ ref, if the initial part of the store is transformed in context $\Gamma$ and $\Gamma \vdash v: D$. In the sequel we abuse the notation and write $\rho$ where a context is expected. For example $\rho \vdash i: D$ means $\Gamma \vdash i: D$ where $\rho$ is interpreted as a context. Store update, notation $\rho[r:=v]$, is the store $\rho^{\prime}, r:=v, \rho^{\prime \prime}$ if $\rho$ is of the form $\rho^{\prime}, r:=v^{\prime}, \rho^{\prime \prime}$ and $\rho^{\prime} \vdash r: D$ ref and $\rho^{\prime} \vdash v: D$.

Index terms are evaluated against a store; evaluation also resolves the distinguished variables size and rank. Assertion $\left(\rho_{1}, i\right) \downarrow^{n, k}\left(\rho_{2}, v\right): D$ abbreviates "index term $i$ of datatype $D$ evaluates under store $\rho_{1}$, size $=n$, and rank $=k$, yielding a value $v$ of datatype $D$ and a new store $\rho_{2}$ ". The rules are straightforward and omitted.

Processes A process $q$ is a pair $(\rho, e)$ composed of a store $\rho$ and an expression $e$. The rule below determines the meaning of assertions of the form $\Gamma \vdash q: T$.

$$
\frac{\Gamma, \rho \vdash e: T}{\Gamma \vdash(\rho, e): T}
$$

A flavor of the process reduction rules is in Figure 7. The remaining rules (for if-false, while-true, while-false, skip, forloop, for-end, and sequential composition) are standard. The rules should be self-explanatory. The let expression evaluates index $i$ to value $v$ and proceeds with expression $e$ with $v$ replacing variable $x$. Since let is a local (process) operation, $x$ cannot be free in $T$, as discussed before. The premises in parenthesis guarantee the good formation of the stores and the expressions involved. Notice that process reduction does not change the type of the expressions involved.

Lemma 4.1 (agreement for process reduction). If $q \rightarrow^{n, k} q^{\prime}$ then $\Gamma^{n, k} \vdash q: T$ and $\Gamma^{n, k} \vdash q^{\prime}: T$.

Lemma 4.2 (process reduction is deterministic). If $q_{1} \rightarrow^{n, k}$ $q_{2}$ and $q_{1} \rightarrow^{n, k} q_{3}$ then $q_{2}=q_{3}$.

The following lemma ensures that processes do not get stuck and will play its part in the main result of the paper.

Lemma 4.3 (progress for processes).

- If $\Gamma^{n, k}, \rho \vdash e$ : skip then $e$ is skip or $(\rho, e) \rightarrow^{n, k} q$.

- If $\Gamma^{n, k}, \rho \vdash i: D$ and $\Gamma^{n, k}, \rho, x: D \vdash e: T$ and $x \notin \mathrm{fv}(T)$ then $(\rho$, let $x: D=i$ in $e) \rightarrow^{n, k} q$.

- If $\Gamma^{n, k}, \rho, p \vdash e_{1}: T$ and $\Gamma^{n, k}, \rho, \neg p \vdash e_{2}: T$ then $\left(\rho\right.$, if $p$ then $e_{1}$ else $\left.e_{2}\right) \rightarrow^{n, k} q$.

- If $\Gamma^{n, k}, \rho, p \vdash e$ : skip then $(\rho$, while $p$ do $e) \rightarrow^{n, k} q$.

- If $\Gamma^{n, k}, \rho, x:\{y:$ int $\mid y \leq i\} \quad \vdash \quad e \quad: \quad T$ then $(\rho$, for $x: i . .1$ do $e) \rightarrow^{n, k} q$.

Programs A program is a vector of processes $q_{1}, \ldots, q_{n}$. Not all such vectors are of interest to us. The following rule is meaning determining for assertions of the form $P: S$.

$$
\frac{\Gamma^{n, 1} \vdash q_{1}: T_{1} \ldots \Gamma^{n, n} \vdash q_{n}: T_{n} \quad T_{1}, \ldots, T_{n}: \text { ptype }}{q_{1}, \ldots, q_{n}: T_{1}, \ldots, T_{n}}
$$

We can easily write the finite differences algorithm in our language. In fact, we can write it in an SPMD or in a MPMD style. In the former case, we follow the C+MPI program in Figure 1. In the latter case we prepare three different expressions: for rank 1 , for rank size, and a third for all the intermediate ranks. The fundamental observation is that all four programs have equivalent types, under the appropriate value for the rank variable.

Program reduction Figure 8 contains an excerpt of the reduction rules for programs. Program reduction is composed of message passing - send/receive - , five collective barrierlike rules-reduce, scatter, gather, broadcast, and val-, one rule for collective decisions, and one rule that provides for local process reduction. As in the previous cases, the premises to the rule may be divided in two parts: those governing the reduction process itself, and those guaranteeing the good formation of the programs involved. The latter are enclosed in parenthesis, as before.

Notation $i \downarrow^{n} v$ abbreviates the evaluation of an int index term under the empty store, $(\varepsilon, i) \downarrow^{n}(\varepsilon, v)$ : int. The proviso, in all rules, that types and datatypes do not contain ref datatypes impedes reference passing (and the associated problem of dangling references at the receiving process). A similar reason forbids the rank variable in types, for this variable has a different value in each different process.

The rule for message-passing, evaluates both index terms in both the send and the receive process. There is a fundamental difference between the first and the second parame- 


$$
\begin{aligned}
& i_{l} \downarrow^{n} m \quad\left(\rho_{l}, i_{l}^{\prime}\right) \downarrow^{n, l}\left(\rho_{l}^{\prime}, v\right): D \quad i_{m} \downarrow^{n} l \quad\left(\rho_{m}, i_{m}^{\prime}\right) \downarrow^{n, m}\left(\rho_{m}^{\prime}, r\right): D \text { ref } \quad(l \neq m) \\
& \left(\Gamma^{n, l} \vdash e_{l}: T\right) \quad\left(\Gamma^{n, m} \vdash e_{m}: T\right) \quad\left(\Gamma^{n, k} \vdash q_{k}: T\right) \quad(k=1 . . n, k \neq l, m) \\
& q_{1}, \ldots, q_{l-1},\left(\rho_{l}, \text { send } i_{l} i_{l}^{\prime} ; e_{l}\right), q_{l+1}, \ldots, q_{m-1},\left(\rho_{m}, \text { receive } i_{m} i_{m}^{\prime} ; e_{m}\right), q_{m+1} \ldots, q_{n} \rightarrow \\
& q_{1}, \ldots, q_{l-1},\left(\rho_{l}^{\prime}, e_{l}\right), q_{l+1}, \ldots, q_{m-1},\left(\rho_{m}^{\prime}[r:=v], e_{m}\right), q_{m+1} \ldots, q_{n} \\
& \frac{i_{k} \downarrow^{n} l \quad\left(\rho_{l}, i_{l}^{\prime}\right) \downarrow^{n, l}\left(\rho_{l}^{\prime}, v\right): D \quad\left(\Gamma^{n} \vdash 1 \leq i_{k} \leq n \text { true }\right) \quad\left(\Gamma^{n, k}, \rho_{k} \vdash i_{k}^{\prime}: D\right) \quad\left(\Gamma^{n, k}, x: D, \rho_{k} \vdash e_{k}: T\right) \quad(k=1 . . n)}{\left(\rho_{k}, \text { let } x: D=\text { broadcast } i_{k} i_{k}^{\prime} \text { in } e_{k}\right)_{k=1}^{n} \rightarrow\left(\rho_{k}, e_{k}\{v / x\}\right)_{k=1}^{l-1},\left(\rho_{l}^{\prime}, e_{l}\{v / x\}\right),\left(\rho_{k}, e_{k}\{v / x\}\right)_{k=l+1}^{n}} \\
& \frac{q_{l} \rightarrow^{n, l} q_{l}^{\prime}\left(\Gamma^{n, k} \vdash q_{k}: T_{k}\right) \quad\left(T_{1}, \ldots, T_{n}: \text { ptype }\right) \quad(k=1 . . n)}{q_{1}, \ldots, q_{n} \rightarrow q_{1}, \ldots, q_{l-1}, q_{l}^{\prime}, q_{l+1}, \ldots, q_{n}}
\end{aligned}
$$

In all rules, $D$ and $T$ contain no ref types and rank $\notin \mathrm{fv}(D, T)$

Figure 8. Program reduction (excerpt)

ter in both cases. The first describes a process rank (target or source), the second the value to be passed, or the reference to hold the result. In general, index terms that denote process ranks cannot refer to the store, for these exact indices show up in the type of the processes (message $m i_{l} D$, in the send case). In such cases we use the abbreviated evaluation, as in $i_{l} \downarrow^{n} m$. In all other cases, we use evaluation under a generic store, as in $\left(\rho_{l}, i_{l}^{\prime}\right) \downarrow^{n, l}\left(\rho_{l}^{\prime}, v\right): D$. The send/receive processes reduce to skip (the stores evolve accordingly); the others remain unchanged. In the rule for broadcast we follow a slightly different strategy. Since a value is transmitted to all processes, the broadcast expression features an explicit continuation, allowing one to substitute the value directly in the continuation process $e_{k}$ (and in its type $T$ ), as opposed to using references.

Main results We are finally in a position to state our main results.

Theorem 4.4 (agreement for program reduction). If $P_{1} \rightarrow$ $P_{2}$ then $P_{1}: S_{1}$ and $P_{2}: S_{2}$.

Program reduction is Church-Rosser. As usual this does not mean that it is strongly normalising: taking advantage of while-loops, processes may engage in infinite computations.

Theorem 4.5 (program reduction is Church-Rosser). If $P_{1} \rightarrow P_{2}$ and $P_{1} \rightarrow P_{3}$ then $P_{2} \rightarrow P_{4}$ and $P_{3} \rightarrow P_{4}$.

In preparation for the progress result, we determine the meaning of assertions of the form $P$ halted using the following rule.

$$
\frac{\left(\rho_{1}: \text { store }\right) \ldots \quad\left(\rho_{n}: \text { store }\right)}{\left(\rho_{1}, \text { skip }\right), \ldots,\left(\rho_{n}, \text { skip }\right) \text { halted }}
$$

We are finally in a position to establish our progress result.

Theorem 4.6 (progress for programs). If $P_{1}: S$ then $P_{1}$ halted or $P_{1} \rightarrow P_{2}$.

\section{Verification of $\mathrm{C}+\mathrm{MPI}$ source code}

This section shows how the theory introduced in Sections 3 and 4 is rendered in VCC, so that C+MPI code may be checked with minimal effort.

The ParTypes VCC library The ParTypes VCC library comprises roughly 800 lines of code and can be obtained from [23]. It comprises:

- The type theory of Section 3 rendered in VCC;

- Contracts for the MPI primitives supported by the theory in Section 4;

- Functions and predicates used in annotations for $\mathrm{C}$ control structures (loops and conditionals) that match protocol control structures (foreach and collective choice); and

- miscellaneous functions and predicates.

In what follows, we outline the contents of the library and complete the section by showing how to annotate our running example so that it can be verified by VCC.

Datatypes in VCC format Index terms, $i$, are $\mathrm{C}$ integer expressions. Propositions, $p$, are $\mathrm{C}$ boolean expressions. Datatypes, $D$, are rendered as a VCC datatype named Data. We can easily show that, for each datatype $D$, there is an equivalent datatype of the form $\{x: B \mid p\}$ where $B$ is a non-refined datatype. Given that VCC does not directly support multi-dimensional arrays, we consider only datatypes of the form $\{x: B \mid p\}$ where $B$ is either int, int array or float array.Such refinements are rendered in VCC as predicates of one (int) or two arguments (arrays). The case for float array refinements corresponds to the following VCC function type definition.

- (ghost

typedef \bool FloatArrayPred[float*][\integer])

VCC verification logic is introduced in $\mathrm{C}$ programs using annotations of the form ${ }_{-}(\ldots)$, and in particular ${ }_{-}$(ghost $\ldots$. . We omit ${ }_{-}(\ldots)$ whenever possible to facilitate readability. We also remove the backslash $(\backslash)$ at the end of lines in $\mathrm{C}$ macros. 
Datatypes $D$ are encoded as follows.

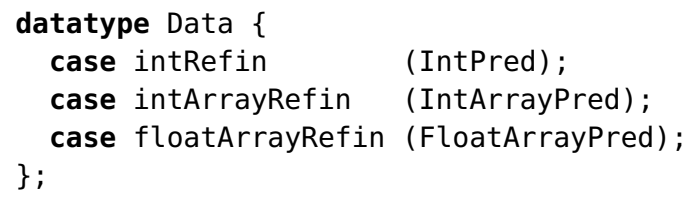

Types in VCC format Types are rendered as a VCC datatype named Protocol. There is one VCC datatype constructor for each type constructor in Figure 3. In addition, dependent types need one different constructor for each Data constructor, for VCC does not support polymorphic type constructors. Following the type formation rules in Figure 3, the constructor for messages, e.g., is a triple of the form ( $\backslash$ integer, $\backslash$ integer, Data). The interesting cases are the dependent constructors: $\forall$, broadcast, and val, for which we use an higher-order abstract syntax (HOAS) [25]. We start by preparing the abstractions for the three basic datatypes that we support:

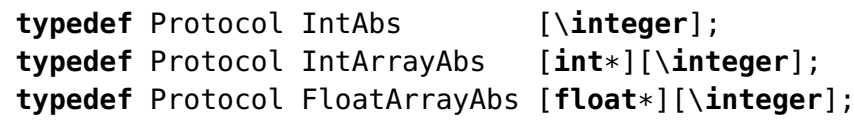

The VCC Protocol datatype may then be defined as follows.

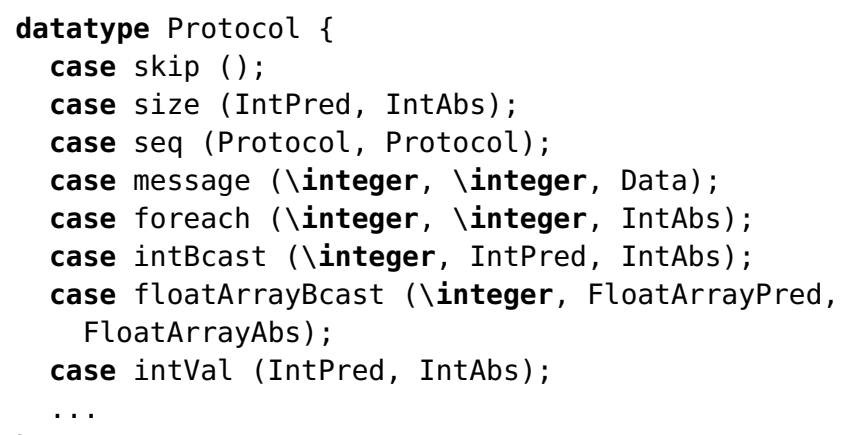

As an example, type $\forall i \leq 10$. message $i(i+1)$ int is rendered in VCC as

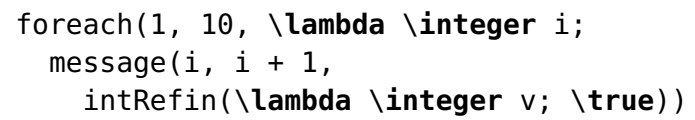

Similarly, type broadcast $1 n$ : int. skip is rendered as

intBcast (1, \lambda \integer v; \true, $\backslash$ lambda \integer $n$; skip())

For the purpose of verifying C+MPI code we are interested in sequents of the form size: $D \vdash T$ : type, for which we prepared a specific constructor, size, in the Protocol datatype.

Type formation (Figure 3) is checked by a dedicated tool [23]. The same tool translates a type $T$ into a VCC Protocol datatype such as the one in Figure 9.

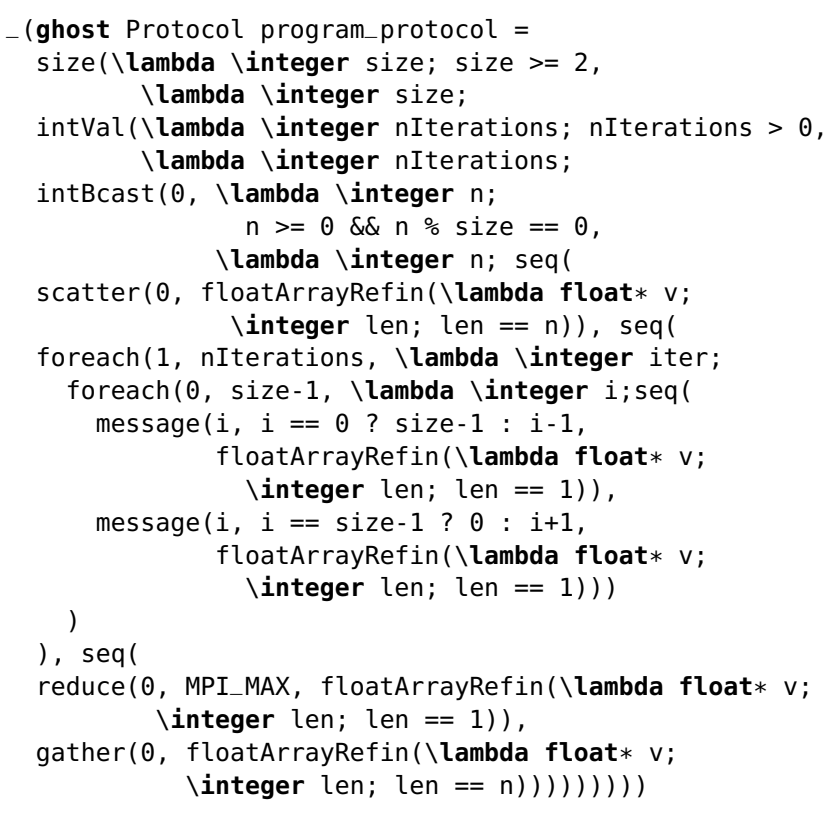

Figure 9. The protocol for finite differences in VCC syntax

Verification flow The flow of program verification can be summarized as follows:

1. The contract for MPI_Init initializes a ghost variable $p$ (of type Protocol) with the protocol the program must follow, such as the one in Figure 9;

2. Contracts for MPI communication primitives progressively match $p$ against the expected communication primitive;

3. Each control structure in the $\mathrm{C}$ program that is related to the protocol is verified, relying on adequate annotations in the body of program;

4. The contract for MPI_Finalize asserts that $p$ is be equivalent to $\operatorname{skip}()$.

In addition to $\mathrm{p}$ above, we use two other ghost variables, size and rank, plus the following machinery:

- A total function, cons, that extracts, from a protocol p, a pair composed of a head and a tail, in such a way that

- $p$ is equivalent to seq (head, tail),

- protocol head is not seq, and

- head is skip only when $p$ is equivalent to skip.

The function accepts rank in addition to a protocol, so that it may decide whether to convert messages to skip. The pair is rendered as a Cons datatype, which we equip with head and tail deconstructors;

- Partial functions that extract from a protocol (typically the head) the various parts of a non-seq Protocol constructor. As an example, for the message $(f, t, d)$ constructor we prepare three functions-messageFrom, 
messageTo, and messageData-returning $f$, $t$, and $d$, respectively; and

- An isSkip ( $p$, rank) predicate defined as $\operatorname{head}(\operatorname{cons}(p$, rank $))==\operatorname{skip}()$.

Contracts for MPI primitives We illustrate the cases of some representative MPI primitives.

MPI_Init declares the ghost variables $p$, rank, and size, and establishes the basic invariant between rank and size. It then calls function cons to extract from protocol $p$ a pair $c$ composed of the head and the tail. The head of $c$ is supposed to be a size type, so we use the two deconstructors for size, namely sizePred and sizeAbs, to extract the predicate and the continuation of the type. The predicate is applied to size (VCC notation: pred [size]) as well as to the continuation of the protocol abs, in line with HOAS.

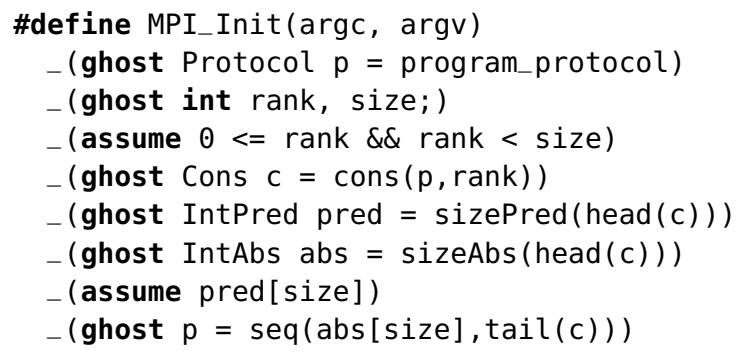

MPI_Comm_rank propagates the constraints introduced for variable rank at MPI_Init to the actual variable in the source code.

\#define MPI_Comm_rank(comm, my_rank)

- (assume rank $=*($ my_rank $))$

MPI_Comm_size does the same to ghost variable size. Once we are done with the verification of MPI operations, we can check that the protocol is reduced to skip:

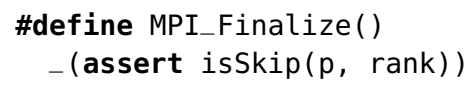

We now exemplify the contract for one of the MPI communication primitives. At MPI_send we check the conformance of the $\mathrm{C}$ code against the type, namely on what concerns the three components of the type: from, to, and the data. As in the case of MPI_Init, the macro uses function cons to extract from the protocol its head/tail pair. Since the head is supposed to be a message type, we use the three deconstructors for message, namely messageFrom, messageTo, and messageDate, to extract the components. These components are then asserted against the expect values in the protocol. In the end we "advance" the protocol to its tail. The simplified version when sending an integer array is as follows.

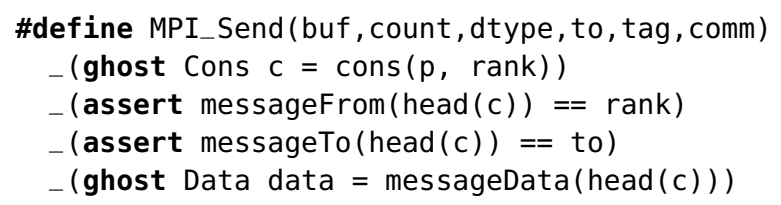

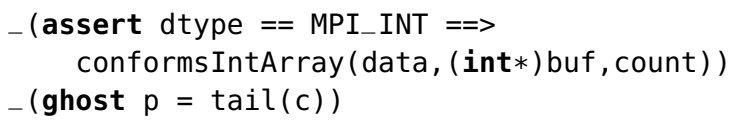

In order to check that the source code conforms to the data part of the message, we use the following predicate:

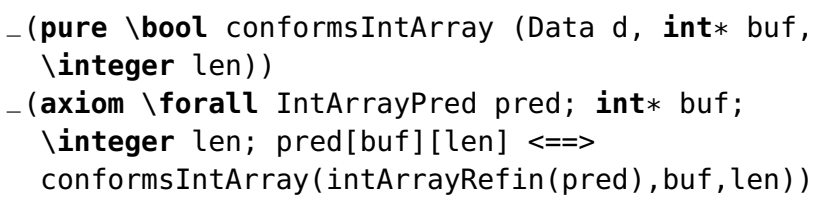

Annotating control flow Annotations are required for C code that matches primitive recursion (foreach), collective choice (if-else), and the val protocol. Four cases arise:

- A val is matched against some $\mathrm{C}$ expression;

- A foreach is matched against a $\mathrm{C}$ for loop, e.g., the outer foreach in Figure 2 when matched against the loop starting at line 9, Figure 1;

- A foreach is matched against an $n$-branched $\mathrm{C}$ conditional, e.g., the inner foreach when matched against the C code in lines 10-25, Figure 1;

- A collective choice matched against a $\mathrm{C}$ conditional.

We analyze the first three cases; the fourth one is similar to the second. Line 2 in the finite differences protocol (Figure 2), namely

val nIterations: positive

requires an annotation of the form

applyInt (NUM_ITER)

to be placed in the source code somewhere after MPI_Init and before MPI_Broadcast. The applyInt macro "injects" the value into the protocol as follows. Once again, the macro uses function cons to extract from the protocol its head/tail pair. The head is now supposed to be a val type; we use the two deconstructors for val, namely intValPred and intValAbs, to extract the predicate and the continuation of the type. The predicate is asserted at value NUM_ITER; value NUM_ITER is further applied to the continuation of the protocol (cf. MPI_Init above). In this case, the macro expands to the following code.

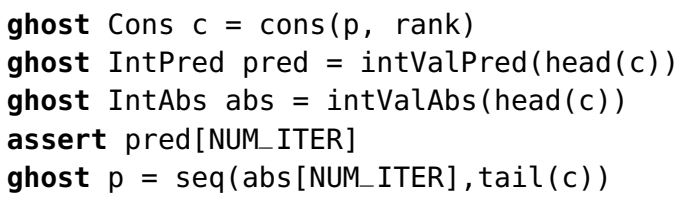

As an example of a foreach protocol that must be matched against a for loop, consider the code in Figure 1, line 9,

for (iter=1; iter <= NUM_ITER; iter ++ )

matched against the foreach protocol in Figure 2, line 5: 


\section{foreach iter: $1 \ldots$ nIterations}

We again start by using function cons to extract from protocol $p$ a pair $c$ composed of the head and the tail of the protocol. The head of $\mathrm{c}$ is now supposed to be a foreach type. We use the deconstructors-foreachLower, foreachUpper, and foreachBody - to extract the three components. We assert the loop boundaries 1 and NUM_ITER against the corresponding values of foreachLower and foreachUpper. For the body of the loop we set $p$ to foreachBody and, at loop exit, assert that the protocol must have been reduced to skip. Finally, for the loop continuation we set $p$ to the tail of the original protocol. All this is rendered as follows.

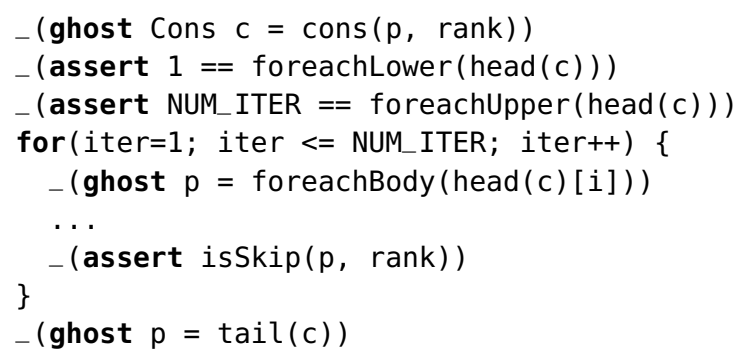

Finally, as an example of a foreach protocol that is supposed to be matched against an if-else conditional, consider the fragment of our running example where each process sends a message to its right and left process, that is, lines 10-25 in Figure 1. The type is in Figure 2, line 6:

foreach $i$ : $0 .$. size-1

The general outline is as above: extract from $p$ the head and the tail, and, at the end of the conditional, set $p$ to the tail of the original protocol. The difference is that in the above case we have a loop body, whereas now, instead, there are several conditional branches. They are all treated alike. In each branch we, intuitively, unfold the foreach and take into account (in sequence) only the foreach body terms that are different from skip. At the end of each branch we assert that $p$ has reduced to skip, and that all other foreach body terms are equivalent to skip. For example, in the first branch, the non-skip elements in the sequence are when $i$ is rank (that of the two MPI_Send, lines 11 and 12), right (that of the first MPI_Recv, line 13), or left (that of the second MPI_Recv, line 14), in this order. We set $p$ as the sequence of applying the foreachBody body to these three values and, at the end, check that $p$ is equivalent to skip. Additionally, we assert that the only non-skip terms in the foreach expansion are exactly when $i$ is rank, right, and left. The annotated code is as follows.

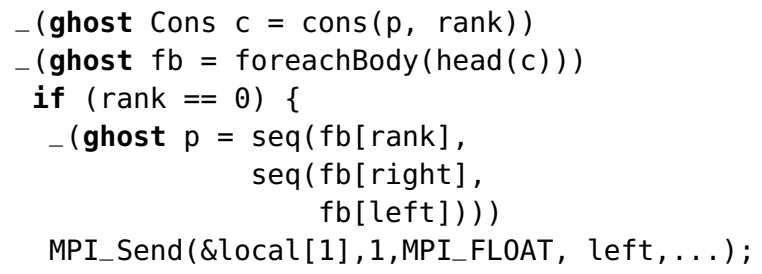

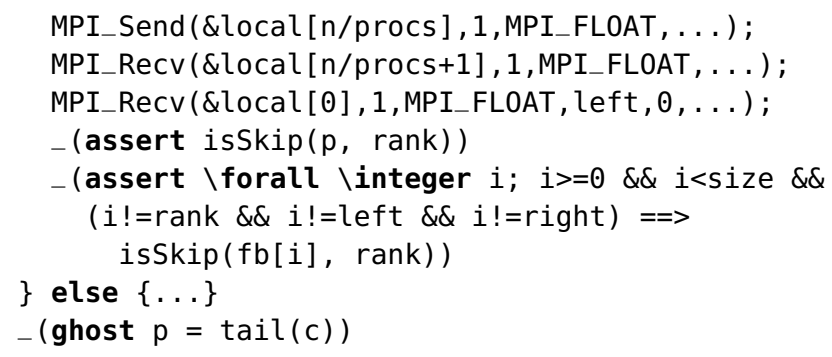

\section{Evaluation}

This section provides an evaluation of the ParTypes approach. We performed a comparative analysis of ParTypes against state-of-the-art MPI verifiers with similar safety guarantees, by measuring the verification times of all tools with varying parameterizations, under a similar environments. We also provide complementary results regarding the ParTypes protocol compiler.

Tools under test For a comparative analysis we considered the following tools:

TASS A model checker which uses symbolic execution [29];

ISP A dynamic verifier that employs dynamic partial order reduction to select the relevant process schedules [24];

MUST A dynamic verifier that employs a graph-based deadlock detection approach [13].

Even though all these tools are able to check deadlocks and type/communication problems, they address the problem of software verification in very distinct ways. Our tool and TASS statically verify source code, while ISP and MUST monitor program execution. TASS relies on model checking and symbolic execution to prove (or disprove), for instance, program deadlock situations, while our tool uses deductive program verification. ISP and MUST both use PnMPI [27] to intercept MPI calls and build a state that allow them to identify deadlocked situations (among others).

The benchmark suite We consider programs taken from textbooks $[8,12,22]$ and the FEVS suite [31], usually used in MPI benchmark analysis: 1-D heat diffusion simulation [31], finite differences [8], N-body simulation [12], parallel Jacobi equation solver [22], parallel dot product [22], and pi calculation [12]. All programs are iterative except for the parallel dot example, that is, they have a core computation/communication loop that is repeated for a number of iterations. We changed of the parallel dot program to be iterative as well for benchmarking purposes. The protocols for five of the six programs are given below; the sixth is the finite differences in Figure 2.

The Diffusion 1-D program calculates the evolution of the diffusion (heat) equation in one dimension over time.

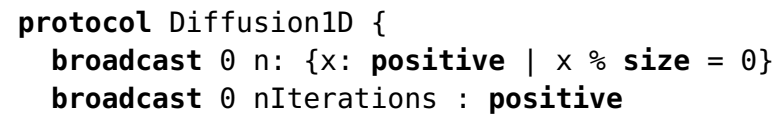




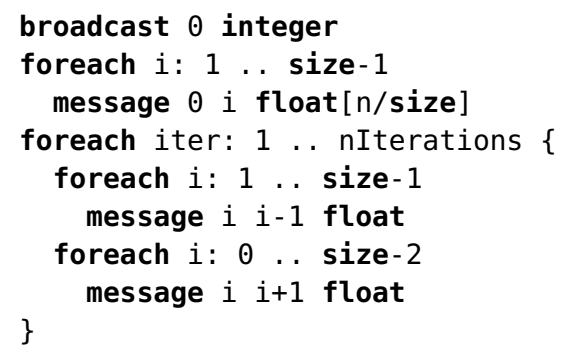

The N-body simulation program simulates a dynamic system of particles under the influence of physical forces.

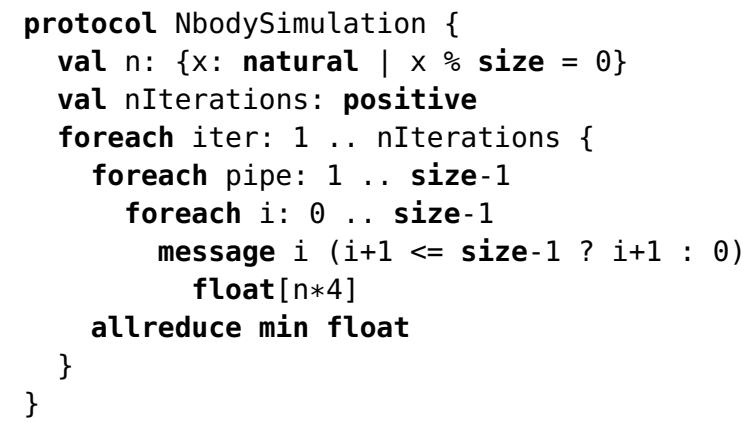

The Parallel Dot program calculates the dot product of two vectors.

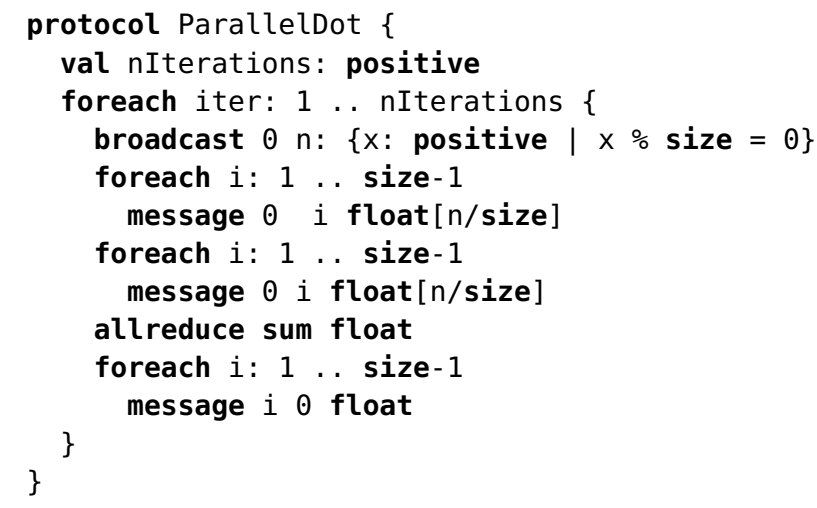

The Parallel Jacobi program solves linear systems of equations using Jacobi's method. ${ }^{2}$

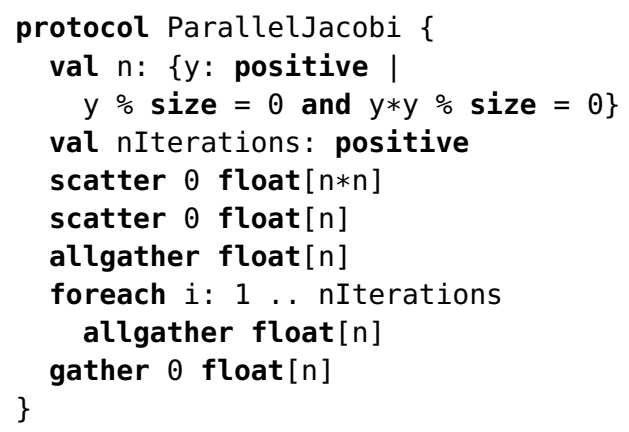

\footnotetext{
${ }^{2}$ SMT solver Z3, used by the ParTypes protocol compiler and VCC, cannot infer $y * y \%$ size $=0$ from $y \%$ size $=0$. Thus we need to state the second condition explicitly.
}

Finally, the Pi program approximates $\pi$ through numerical integration.

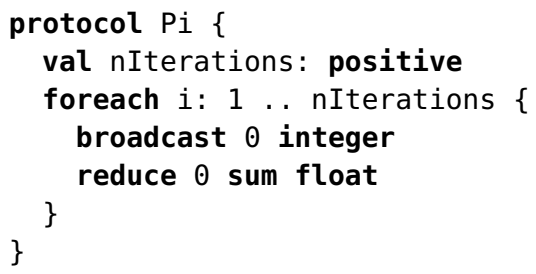

Experimental setup For each benchmark program, we stripped all computation code that does not affect the program's behavior in terms of MPI calls made or their arguments. In this manner, we can measure the verification effort strictly in terms of MPI interactions. This approach rules out the overhead associated with computation code that comes from actual execution in the case of ISP and MUST, symbolic execution in the case of TASS, and verification of memory accesses by VCC for ParTypes. We fixed buffer sizes used by programs in communication to the minimum value necessary for the programs to work correctly. We then prepared two annotated versions of each program, one for ParTypes and the other for TASS. TASS requires annotations for the input parameters of a program, including the number of processes and the number of loop iterations. No annotations are required for ISP and MUST, since both tools execute the target program directly.

For benchmarking we use a Ubuntu 14.04 Linux machine with 64 GB of RAM and 4 AMD Opteron 6376 processors, each with 16 cores, totalling 64 cores. The MPI runtime (required for ISP and MUST) is MPICH 3.0 and the Java runtime (for TASS) is Oracle's JRE 1.8. Tools ISP 0.3, MUST 1.4, and TASS 1.2 all run natively in this machine. VCC 2.3, supported by Z3 3.2 [? ], runs on a hosted Windows 7 virtual machine using a KVM/QEMU 2.0 hypervisor. Note that VCC runs only on Windows platforms; in spite of the virtualisation overhead, we tried to make the VCC setup as close as possible to that used for all other tools.

We run each tool varying the number of processes or the number of iterations for ISP, MUST, and TASS. These variations are not required for ParTypes. The first set of results resulted from varying the number of processes from 2 to 32 . We do not consider 64 processes (the number of available cores in the host machine) since ISP and MUST use an extra process for runtime monitoring in addition to one MPI process per core. In conjunction, the number of iterations is fixed to $2^{8}$ for ISP and TASS, and $4^{8}$ for MUST. For the second set of results, we fix the number of processes to 32 , and let the number of iterations range from $2^{0}$ to $2^{8}$ for ISP and TASS, and from $4^{0}$ to $4^{8}$ for MUST. The higher values for MUST are necessary in order to obtain a reasonable analysis of the scalability trend.

Under this setup, verification times are taken following the start-up performance methodology of [9]. For each benchmark and configuration of parameters, we took 31 

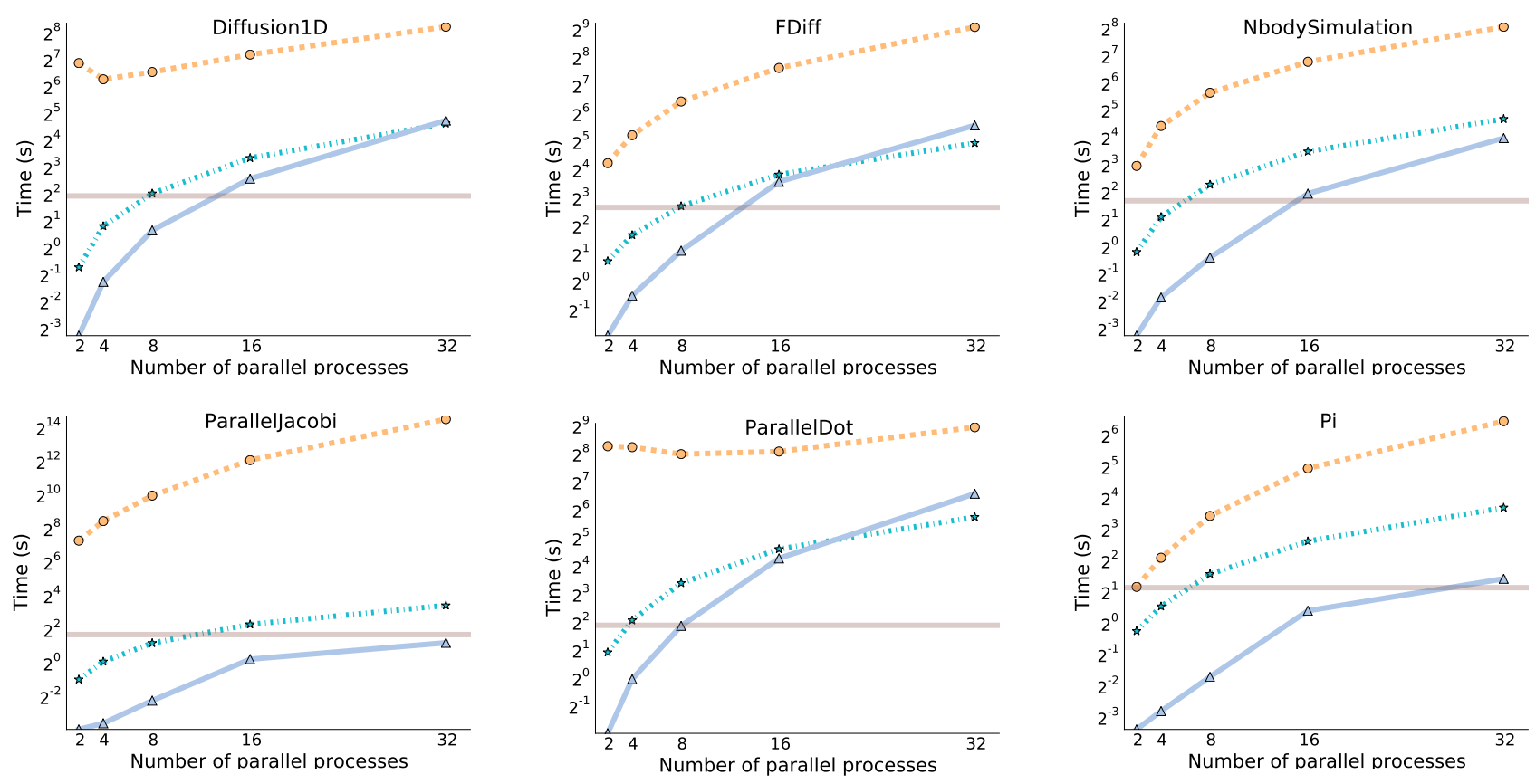

- ParTypes o= OTASS $\triangle \triangle \mathrm{SSP}$ \#MUST

Figure 10. Results for the experiments varying the number of processes
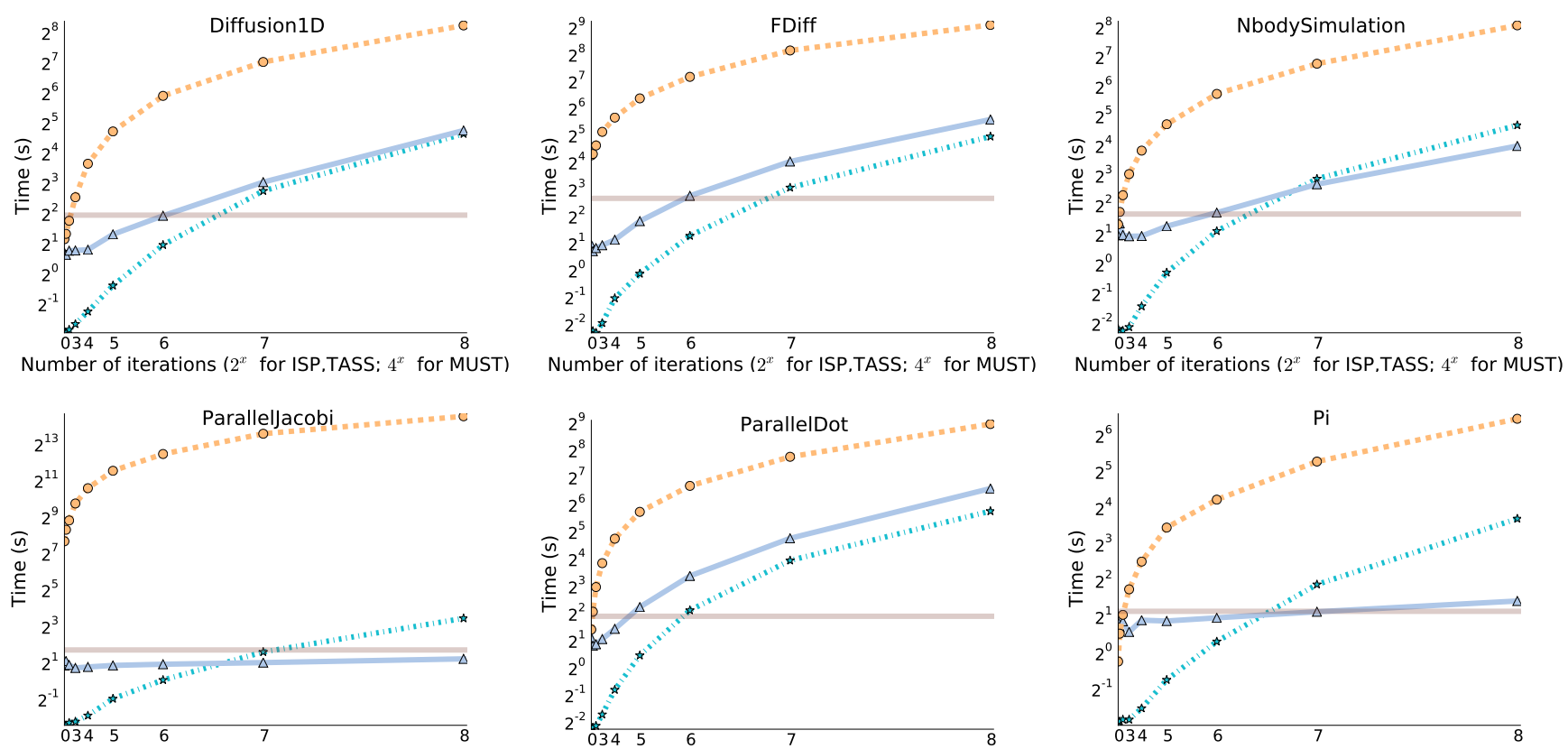

Number of iterations ( $2^{x}$ for ISP,TASS; $4^{x}$ for MUST)

Number of iterations ( $2^{x}$ for ISP,TASS; $4^{x}$ for MUST)

Number of iterations ( $2^{x}$ for ISP,TASS; $4^{x}$ for MUST)

-ParTypes O=OTASS $\triangle \triangle \triangle S P \quad$ \#MUST

Figure 11. Results for the experiments varying the number of loop iterations 
samples of the verification time. We discard the first sample, and compute the average verification time using the remaining 30 samples.

Results The results are depicted in Figure 10 (for a variable the number of processes) and Figure 11 (for a variable number of iterations). All plots in each figure, one per benchmark program, have a linear scale for the $x$ axis (process or iteration count) and a log- 2 scale for the $y$ axis (verification time in seconds). Each plot shows the verification times of ISP, MUST, and TASS versus ParTypes. The ParTypes verification time is shown constant, since it does not depend on the choice of values for the number of processes or the number of iterations.

As the number of processes and iterations grow larger, the ParTypes verification time can be (sometimes several) orders of magnitude lower than that of the other tools (particularly TASS). This trend is observable in all results, except for ISP in the case of ParallelJacobi and Pi, two programs that only use collective communication primitives. Our approach is immune to the growth of the number of processes or the number of iterations, in clear contrast with the remaining tools under test.

Evaluating the protocol compiler Table 1 presents results regarding the protocol compiler that is embedded in the ParTypes Eclipse plugin [23]. We evaluated the command-line version of the tool on the machine described above, by measuring the time it takes to validate a protocol and translate it to VCC form. For each protocol in our benchmark suite the table lists the total execution time, the protocol's validation time, the portion of time spent executing the Z3 SMT solver for proof discharges during validation, and the time elapsed in the final stage of VCC translation. All times are in milliseconds and represent the average of 30 measurements. The results show that the performance is essentially dominated by the time spent in Z3.

Table 1. Protocol compiler - execution times (ms)

\begin{tabular}{lccc} 
& Total & Validation $(Z 3)$ & VCC translation \\
\hline Diffusion1D & 332 & $320(309)$ & 12 \\
FDiff & 314 & $304(290)$ & 10 \\
NbodySimulation & 185 & $178(167)$ & 7 \\
ParallelJacobi & 229 & $219(206)$ & 10 \\
ParallelDot & 313 & $304(291)$ & 9 \\
Pi & 136 & $131(122)$ & 5
\end{tabular}

\section{Related work}

Tools for the verification of MPI programs A recent survey covers the state-of-the-art in MPI program verification [10], providing a comprehensive overview of the diverse dimensions of verification and of the methodologies employed. Verification may target the validation of arguments to MPI primitives as well as resource usage [34], ensuring interaction properties such as the absence of deadlocks [24, 29, 34], or asserting functional equivalence to sequential programs [29, 31]. Methodologies range from traditional static and dynamic analysis up to model checking and symbolic execution. In comparison, our novel methodology is based on type checking and deductive program verification.

TASS [31, 32] employs model checking and symbolic execution techniques in order to verify a number of safety properties such as deadlock detection, buffer overflows and memory leaks, plus user-specified assertions about the interactive behavior of processes in a MPI program. TASS also also checks functional equivalence between MPI programs and sequential counterparts [31]. CIVL [? ], the recent successor to TASS, employs the same sort of techniques, but uses a unified intermediate verification language that handles not only MPI, but also code written using other popular standards for parallel programming like OpenMP or CUDA.

ISP [24] is a deadlock detection tool that explores all possible process interleavings using a fixed test harness. Other runtime verifiers such as DAMPI [34] or MUST [13], also allow for the detection of deadlocks. MOPPER [6] is a verifier that detects deadlocks by analyzing execution traces of MPI programs. The concept of parallel controlflow graphs [1] provides for the static and dynamic analysis of MPI programs, e.g., as a means to verify sender-receiver matching in MPI source code.

Session type theories Among all theoretical works on session types, the closest to ours is probably that of [4], introducing dependent types and a form of primitive recursion into session types. ParTypes provides for various communication primitives (in contrast to message passing only) and incorporates dependent collective choices. On the other hand, we do not allow session delegation. At the term level, we work with a while language, as opposed to a variant of the the $\pi$-calculus. Kouzapas et al. introduce a notion of broadcast in the setting of session types [17]. A new operational semantics system provides for the description of 1-to$n$ and $n$-to- 1 message passing, where $n$ is not fixed a priori, meaning that a non-deterministic number of processes may join the operation, the others being left waiting. Types, however, do not distinguish point-to-point from broadcast operations. We work on a deterministic setting and provide a much richer choice of type operators.

Scribble Based on the theory of multiparty session types [14], Scribble [15? ? ? ] is a language to describe protocols for message-passing programs. Protocols written in Scribble include explicit senders and receivers, thus ensuring that all senders have a matching receiver and vice versa. Global protocols are projected into each of their participants' counterparts, yielding one local protocol for each participant present in the global protocol. Developers can then implement programs based on the local protocols and us- 
ing standard message-passing libraries, as in Multiparty Session C [21].

Pabble [20] is a parametric extension of Scribble, which adds indices to participants and represents Scribble protocols in a compact and concise notation for parallel programming. Pabble protocols can represent interaction patterns of MPI programs where the number of participants in a protocol is decided at runtime. Pabble was applied to generate communication safe-by-construction MPI programs [? ], leveraging the close affinity between Pabble protocols and MPI programs. These works show how protocol languages can be used for verifying or constructing MPI programs.

In ParTypes we depart from multiparty session types along two distinct dimensions: 1) our protocol language is specifically built for MPI primitives, and 2) we do not explicitly project a protocol nor generate the MPI code but else check the conformance of code against a global protocol. In contrast to ParTypes, works on parameterised session types [20? ] cannot deal with:

- Protocols where a given communication (say the source or the target) depends on the contents of previously exchanged data;

- Protocols whose behaviour does not depend directly on message passing, but else on a data-dependent common agreement among all processes (what we call collective operations); and

- Most of the collective operations (broadcast, gather, scatter, reduce) primitives, as well as general and array passing.

- We address the verification of real world code, while [4] works on the $\pi$ calculus and is not implemented, and [20] does not check existing code.

Dependent type systems Following Martin-Löf's works on constructive type theory [18], a number of programming languages have made use of dependent type systems. Rather than taking advantage of the power of full dependent type systems (that brings undecidability to type checking), $\mathrm{Xi}$ and Pfenning [36] introduce a restricted form of dependent types, where types may refer to values of a restricted domain, as opposed to values of the term language. The type checking problem is then reduced to constraint satisfiability, for which different tools nowadays are available. Our language follows this approach. Xanadu [35] incorporates these ideas in a imperative C-like language. Omega [28] and Liquid Types [26] are two further examples of pure functional languages that either resort to theorem proving or type inference. All these languages are functional; their type systems cannot abstract program's communication patterns.

Previous work on ParTypes We initially formulated the problem of verifying C+MPI programs using a type-based approach in [? ]. Subsequent work proposes a preliminary evaluation of the approach and experiments [?], where we did not make use of a protocol language, verification did not scale and also required an a priori defined number of processes. We also considered the type-based verification of WhyML parallel programs [? ] and the synthesis of correctby-construction $\mathrm{C}+\mathrm{MPI}$ programs from protocol specifications [?].

\section{Conclusion and future work}

We presented a type-based methodology to statically verify message-passing parallel programs. By checking that a program follows a given protocol, we guarantee a set of safety properties for the program, in particular that it does not run into deadlocks. In contrast to other state-of-the-art approaches that suffer from scalability issues, our approach is insensitive to parameters such as the number of processes, problem size, or the number of iterations of a program.

The limitations of ParTypes can be discussed along two dimensions:

- ParTypes addresses the core messaging primitives in MPI, namely: send/receive, broadcast, scatter/gather, reduce, and allreduce/allgather. Notable exceptions are non-blocking operations and wildcard receive (the ability to receive from any source). State-of-the art static verifiers for MPI (see Section 7) roughly deal with this core. On what concerns control primitives, ParTypes include primitive recursion and collective choice, a novel primitive.

- Our VCC methodology is sound but not complete with respect to the core programming language set forth in Section 3.

In view of these limitations, we plan to address further MPI communication primitives, including non-blocking message passing (the "immediate" operations of MPI) and non-determinism in the form of accepting messages from any source. Furthermore, we plan to take advantage of the rich notion of type equivalence to allow for programs with different control flows to be matched against the same protocol.

The idea of a global protocol that governs a parallel program offers further interesting applications, including support for correct-by-construction code generation, test suite generation, and runtime verification.

Acknowledgments This work is supported by FCT through project Advanced Type Systems for Multicore Programming and project Liveness, Statically (PTDC/EIA$\mathrm{CCO} / 122547$ and 117513/2010); the LaSIGE Research Unit, (UID/CEC/00408/2013), EPSRC EP/K011715/1, EP/K034413/1, and EP/L00058X/1, the Danish Foundation for Basic Research, project IDEA4CPS (DNRF86-10), EU project FP7-612985 UpScale and COST Action IC1201 BETTY. We would like to thank Stephen Siegel and Zheng Manchun for help on using TASS, and Dimitris Mostrous for his insightful comments. 


\section{References}

[1] S. Aananthakrishnan, G. Bronevetsky, and G. Gopalakrishnan. Hybrid approach for data-flow analysis of MPI programs. In ICS, pages 455-456. ACM, 2013.

[2] E. Cohen, M. Dahlweid, M. Hillebrand, D. Leinenbach, M. Moskal, T. Santen, W. Schulte, and S. Tobies. VCC: A practical system for verifying concurrent C. In TPHOLs, volume 5674 of $L N C S$, pages 23-42. Springer, 2009.

[3] T. Coquand. Logical Frameworks, chapter An algorithm for testing conversion in type theory. CUP, 1991.

[4] P. Deniélou, N. Yoshida, A. Bejleri, and R. Hu. Parameterised multiparty session types. Logical Methods in Computer Science, 8(4), 2012.

[5] FEVS. A functional equivalence verification suite, 2015. http://vsl. cis.udel. edu/fevs/.

[6] V. Forejt, D. Kroening, G. Narayanswamy, and S. Sharma. Precise predictive analysis for discovering communication deadlocks in MPI programs. In FM, volume 8442 of $L N C S$, pages 263-278. Springer, 2014.

[7] M. Forum. MPI: A Message-Passing Interface StandardVersion 3.0. High-Performance Computing Center Stuttgart, 2012.

[8] I. Foster. Designing and building parallel programs. AddisonWesley, 1995.

[9] A. Georges, D. Buytaert, and L. Eeckhout. Statistically rigorous Java performance evaluation. In OOPSLA, pages 57-76. ACM, 2007.

[10] G. Gopalakrishnan, R. Kirby, S. Siegel, R. Thakur, W. Gropp, E. Lusk, B. D. Supinski, M. Schulz., and G. Bronevetsky. Formal analysis of MPI-based parallel programs. CACM, 54 (12):82-91, 2011.

[11] A. D. Gordon and C. Fournet. Principles and applications of refinement types. In Logics and Languages for Reliability and Security, pages 73-104. IOS Press, 2010.

[12] W. Gropp, E. Lusk, and A. Skjellum. Using MPI: portable parallel programming with the message passing interface. MIT press, 1999.

[13] T. Hilbrich, J. Protze, M. Schulz, B. R. de Supinski, and M. S. Müller. MPI runtime error detection with MUST: advances in deadlock detection. In SC, page 30. IEEE/ACM, 2012.

[14] K. Honda, N. Yoshida, and M. Carbone. Multiparty asynchronous session types. In POPL, pages 273-284. ACM, 2008.

[15] K. Honda, A. Mukhamedov, G. Brown, T. Chen, and N. Yoshida. Scribbling interactions with a formal foundation. In ICDCIT, volume 6536 of $L N C S$, pages 55-75. Springer, 2011.

[16] Y. Huang, E. Mercer, and J. McCarthy. Proving MCAPI executions are correct using SMT. In ASE, pages 26-36. IEEE, 2013.

[17] D. Kouzapas, R. Gutkovas, and S. J. Gay. Session types for broadcasting. In PLACES, volume 155 of EPTCS, pages 2531, 2014.

[18] P. Martin-Löf. Intuitionistic Type Theory. Bibliopolis-Napoli, 1984.
[19] N. Ng and N. Yoshida. Pabble: Parameterised Scribble for Parallel Programming. In PDP, pages 707-714. IEEE Computer Society, 2014.

[20] N. Ng and N. Yoshida. Pabble: parameterised Scribble. SOCA, pages 1-16, 2014.

[21] N. Ng, N. Yoshida, and K. Honda. Multiparty Session C: Safe parallel programming with message optimisation. In TOOLS Europe, volume 7304 of LNCS, pages 202-218. Springer, 2012.

[22] P. Pacheco. Parallel programming with MPI. Morgan Kaufmann, 1997.

[23] ParTypes. Partypes web site. http://gloss.di.fc.ul.pt/ParTypes.

[24] S. Pervez, G. Gopalakrishnan, R. M. Kirby, R. Palmer, R. Thakur, and W. Gropp. Practical model-checking method for verifying correctness of MPI programs. In PVM/MPI, volume 4757 of LNCS, pages 344-353. Springer, 2007.

[25] F. Pfenning and C. Elliot. Higher-order abstract syntax. SIGPLAN Not., 23(7):199-208, 1988. ISSN 0362-1340.

[26] P. M. Rondon, M. Kawaguchi, and R. Jhala. Liquid types. In PLDI, pages 159-169. ACM, 2008.

[27] M. Schulz and B. R. de Supinski. A flexible and dynamic infrastructure for MPI tool interoperability. In ICPP'06, pages 193-202. IEEE, 2006.

[28] T. Sheard and N. Linger. Programming in Omega. In CEFP, volume 5161 of $L N C S$, pages 158-227. Springer, 2007.

[29] S. Siegel and G. Gopalakrishnan. Formal analysis of message passing. In VMCAI, volume 6538 of $L N C S$, pages 2-18. Springer, 2011.

[30] S. Siegel and L. Rossi. Analyzing BlobFlow: A case study using model checking to verify parallel scientific software. In EuroPVM/MPI, volume 5205 of LNCS, pages 274-282. Springer, 2008.

[31] S. F. Siegel and T. K. Zirkel. FEVS: A Functional Equivalence Verification Suite for high performance scientific computing. Mathematics in Computer Science, 5(4):427-435, 2011.

[32] S. F. Siegel and T. K. Zirkel. Loop invariant symbolic execution for parallel programs. In VMCAI, volume 7148 of $L N C S$, pages 412-427. Springer, 2012.

[33] S. F. Siegel, M. B. Dwyer, G. Gopalakrishnan, Z. Luo, Z. Rakamaric, R. Thakur, M. Zheng, and T. K. Zirkel. CIVL: The concurrency Intermediate Verification Language. Technical Report UD-CIS-2014/001, Department of Computer and Information Sciences,University of Delaware, 2014.

[34] A. Vo, S. Aananthakrishnan, G. Gopalakrishnan, B. R. de Supinski, M. Schulz, and G. Bronevetsky. A scalable and distributed dynamic formal verifier for MPI programs. In $S C$, pages 1-10. IEEE, 2010.

[35] H. Xi. Imperative programming with dependent types. In LICS, pages 375-387. IEEE, 2000.

[36] H. Xi and F. Pfenning. Dependent types in practical programming. In POPL, pages 214-227. ACM, 1999. 


\section{A. Finite differences in SPMD and MPMD formats}

Protocols can be used for both Single Program Multiple Data and Multiple Program Multiple Data style programs. Figure A presents the finite differences example in a Single Program Multiple Data form, as written in our programming language (cf. running example, written in C+MPI, 1). Figures A to A present the exact same problem, this time written in a Multiple Program Multiple Data form. As we can easily see, the code in each program is the "projection" of that in Figure A to each of the rank categories: first, last, and others. Multiple Program Multiple Data is supported by out theory, but not by $\mathrm{C}+\mathrm{MPI}$. Both examples follow the protocol in Figure 2.

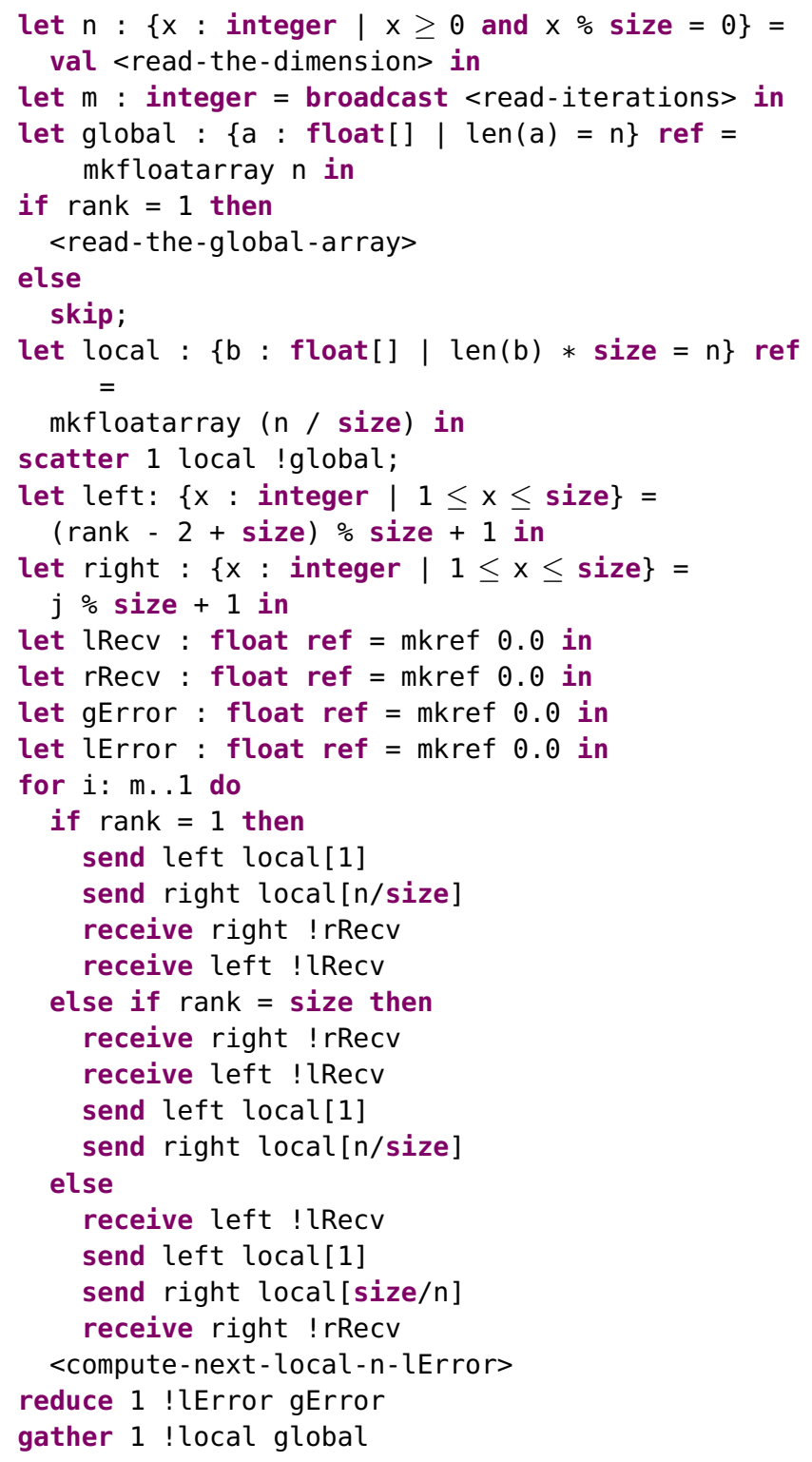

Figure 12. Finite differences in a single program

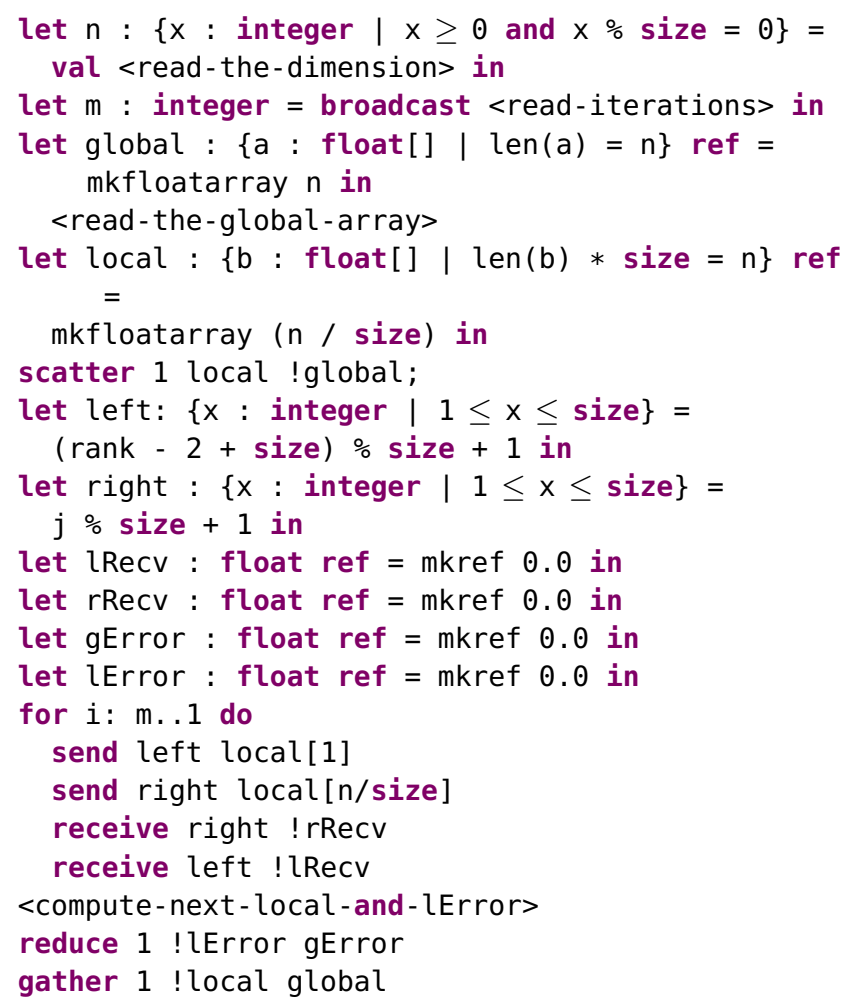

Figure 13. Finite differences in multiple programs (first process)

\section{B. The VCC theory}

This section presents an overview of the type theory when encoded in VCC.

\section{B.1 The Data datatype}

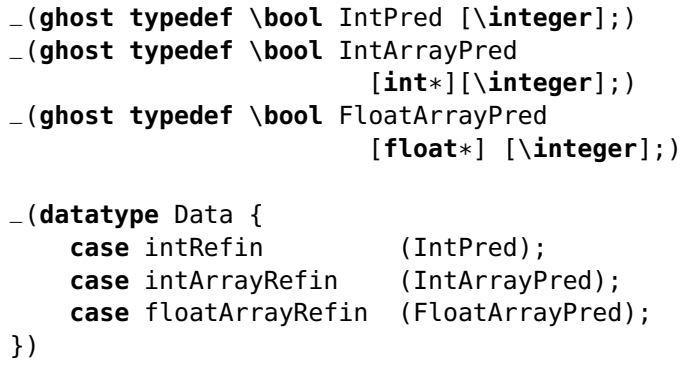

\section{B.2 The Protocol datatype}

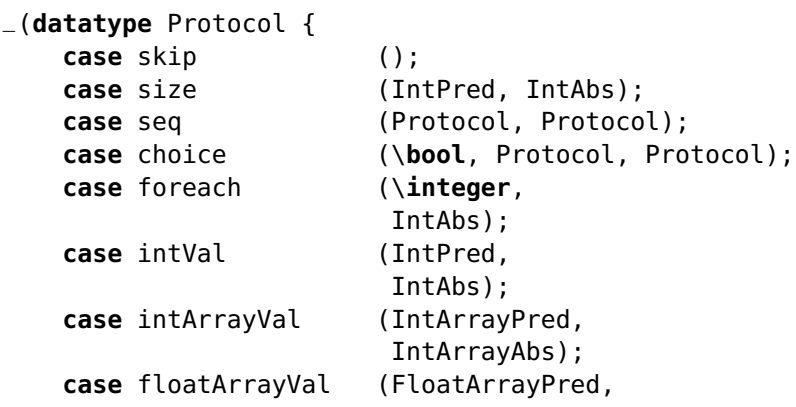




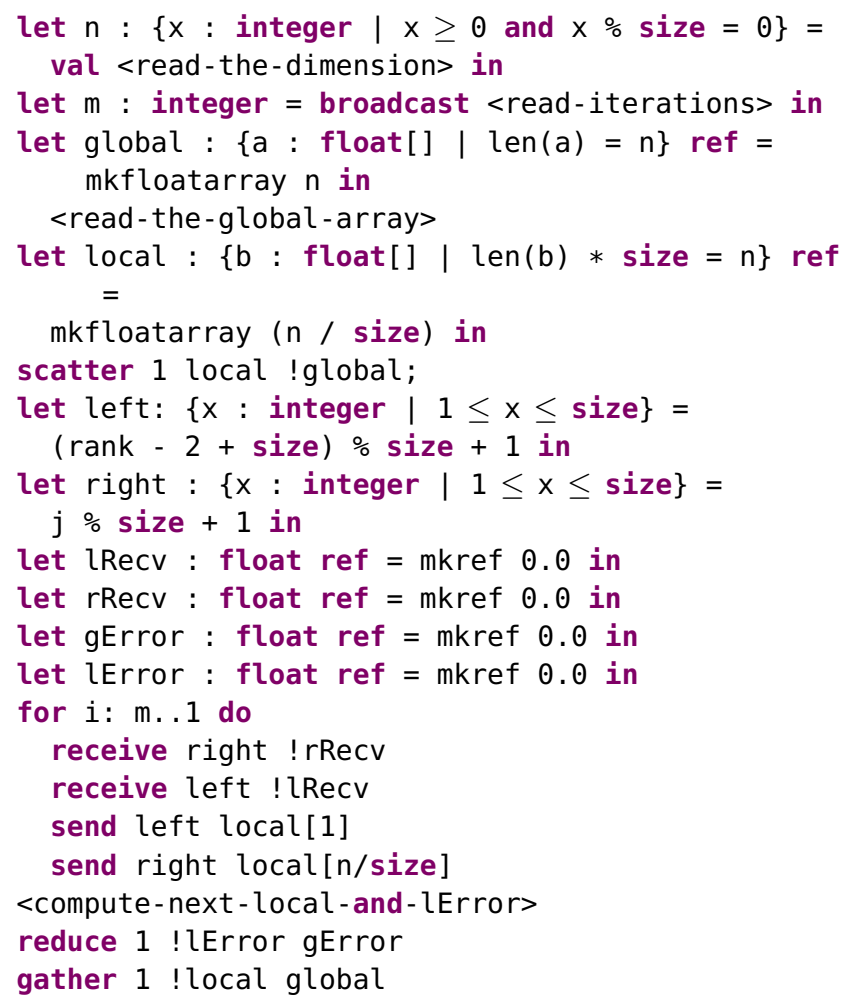

Figure 14. Finite differences in multiple programs (last process)

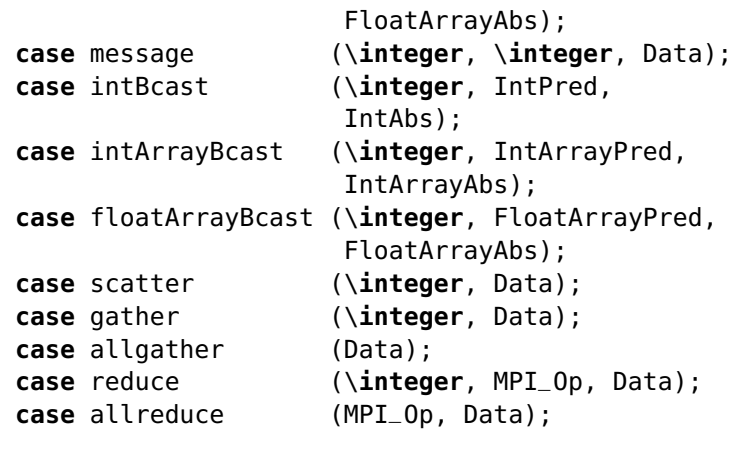

\section{B.3 The cons datatype and the cons funtion}

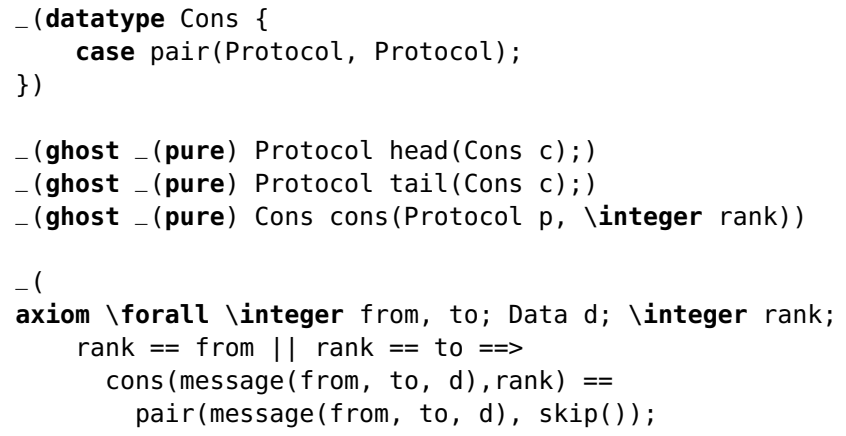

axiom \forall \integer from, to; Data d; \integer rank; rank $!=$ from $\& \&$ rank $!=$ to $==>$

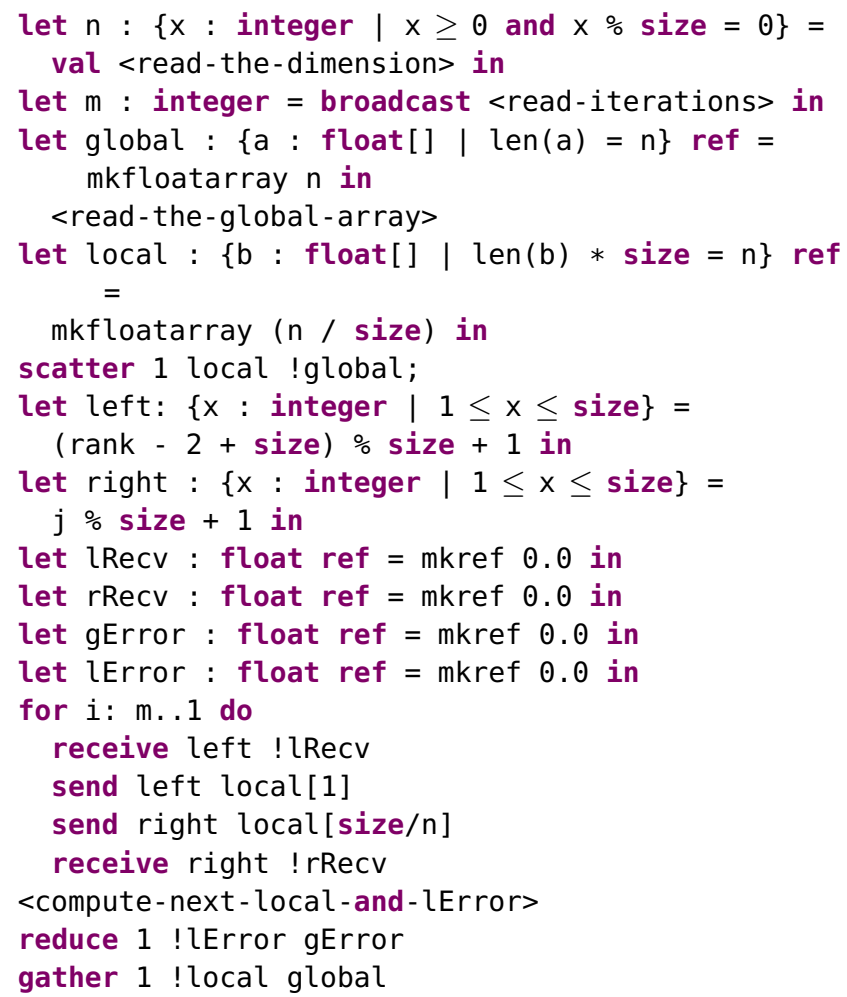

Figure 15. Finite differences in multiple programs (other processes)

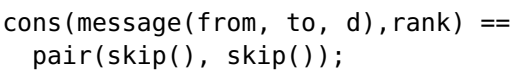

axiom \forall IntPred pred; IntAbs abs; Protocol p; integer rank; cons (size(pred, abs), rank) == pair(size(pred, abs), skip());

axiom \forall IntPred pred; IntAbs abs; \integer rank, root;

cons(intBcast (root, pred, abs), rank) == pair(intBcast(root, pred, abs), skip());

// .. similar for other constructors ...

\section{B.4 Sample contracts for MPI primitives}

\section{B.4.1 MPI_Init and MPI_Finalize}

\#define MPI_Init(argc,argv) \{ 


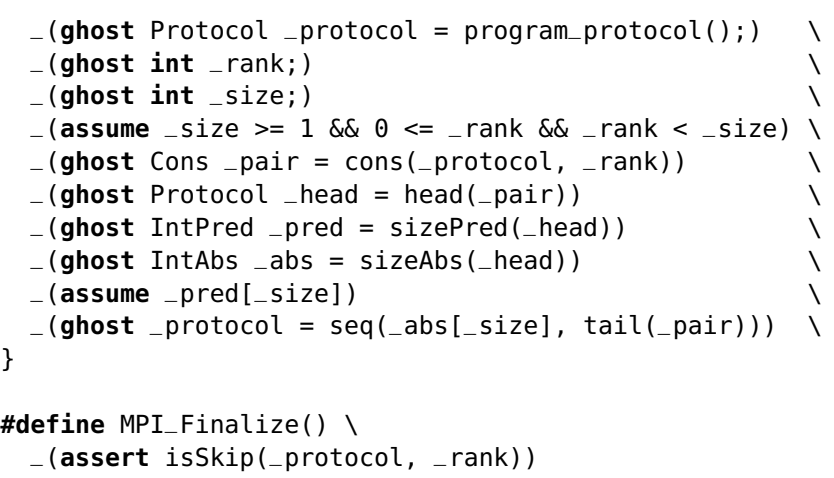

\section{B.4.2 MPI_Send and MPI_Recv}

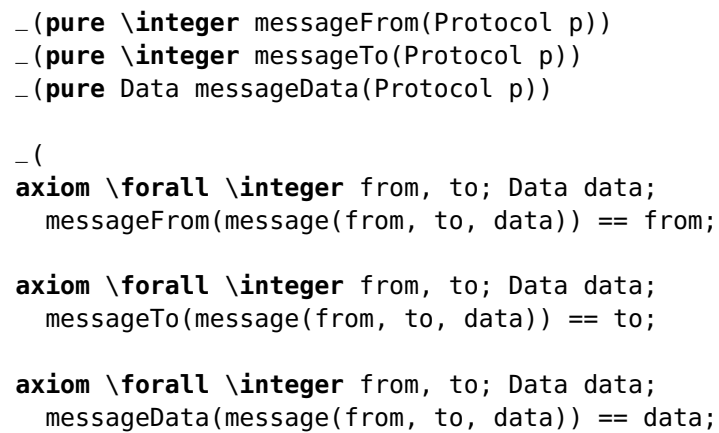

\section{B.4.3 Collective operations (subset)}

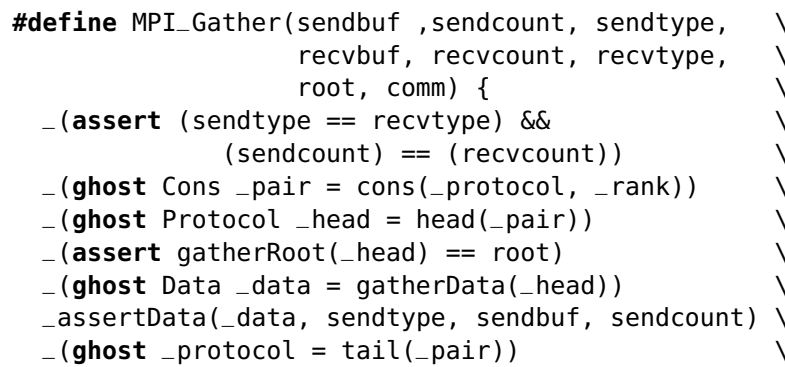

\#define MPI_Scatter(sendbuf, sendcount, sendtype, recvbuf, recvcount, recvtype, root, comm) \{

_ (assert (sendtype $==$ recvtype) $\& \&$ ( sendcount) $==($ recvcount $)$ )

- (ghost Cons_pair = cons (_protocol, _rank))

_ (ghost Protocol _head = head (_pair))

- (assert scatterRoot (_head) == root)

- (ghost Data _data = scatterData (_head))

- (assert rank $==$ root $==>$ -assertData(_data, sendtype, sendbuf, size $*($ sendcount $))$ )

( assume rank $!=$ root $==>$

_assumeData(_data, sendtype, sendbuf, -size * (sendcount)) )

( $($ ghost - protocol $=$ tail $($ - pair $))$

\#define MPI_Reduce(sendbuf, recvbuf, count, dtype, op, root, comm)

_(ghost Cons _pair = cons (_protocol, _rank))

_(ghost Protocol_head $=$ head (_pair))

- (assert reduceRoot $($-head $)==$ root $)$

- (assert reduce0p (_head) == op )

_ (ghost Data_data = reduceData (_head))

_assertData(_data, dtype, sendbuf, count) \}

\section{Complete definitions for the type and the programming languages}

This section introduces the complete definitions for the type system and the programming language.

Type formation rules The axioms and inference rules for deciding on what counts as a type, a datatype, a proposition, an index term and a context, are in Figure 16. The rules for datatype subtyping are in Figure 17.

Type equality The complete set of inference rules for deciding on when two types are equivalent is presented in Figure 18 .

Algorithmic type equality is composed of two relations: Structural Congruence (Figure 19) and Type Conversion (Figure 20).

Reference formation The rule for references are presented in Figure 21. These rules extend those in Figure 16.

Expression formation The typing rules for deciding what counts as an expression are presented in Figure 22.

Store formation Stores map reference identifiers to values. The formation rules are presented in Figure 23.

Index evaluation Figure 24 introduces the assertion rules used for index evaluation.

Process reduction Figure 25 presents the process reduction rules. 
Type formation, $\Gamma \vdash T$ : type

$$
\begin{aligned}
& \frac{\Gamma: \text { context }}{\Gamma \vdash \text { skip : type }} \\
& \frac{\Gamma \vdash 1 \leq i_{1}, i_{2} \leq \text { size } \wedge i_{1} \neq i_{2} \text { true } \quad \Gamma \vdash D: \text { dtype }}{\Gamma \vdash \text { message } i_{1} i_{2} D: \text { type }} \\
& \Gamma \vdash 1 \leq i \leq \text { size true } \quad \Gamma, x: D \vdash T: \text { type } \\
& \overline{\Gamma \vdash \text { reduce } i \text { : type }} \quad \overline{\Gamma \vdash \operatorname{val} x: D \cdot T: \text { type }} \\
& \Gamma \vdash 1 \leq i \leq \text { size true } \Gamma \vdash D<: D^{\prime} \text { array } \\
& \Gamma \vdash \text { scatter } i D \text { : type } \\
& \Gamma \vdash 1 \leq i \leq \text { size true } \quad \Gamma \vdash D<: D^{\prime} \text { array } \\
& \Gamma \vdash \text { gather } i D: \text { type } \\
& \underline{\Gamma, x:\{y: \text { int } \mid y \leq i\} \vdash T: \text { type }} \quad \underline{\Gamma \vdash T_{1}: \text { type } \quad \Gamma \vdash T_{2}: \text { type }}
\end{aligned}
$$

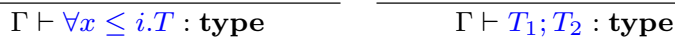

$$
\begin{aligned}
& \Gamma \vdash 1 \leq i \leq \text { size true } \quad \Gamma, x: D \vdash T: \text { type } \\
& \Gamma \vdash \text { broadcast } i x: D . T \text { : type } \\
& \frac{\Gamma \vdash p: \text { prop } \Gamma \vdash T_{1}: \text { type } \quad \Gamma \vdash T_{2}: \text { type }}{\Gamma \vdash p ? T_{1}: T_{2}: \text { type }}
\end{aligned}
$$

Datatype formation, $\Gamma \vdash D:$ dtype

$$
\begin{array}{cc}
\frac{\Gamma: \text { context }}{\Gamma \vdash \text { int }: \text { dtype }} & \frac{\Gamma: \text { context }}{\Gamma \vdash \text { float }: \text { dtype }} \\
\frac{\Gamma \vdash D: \text { dtype }}{\Gamma \vdash D \text { array : dtype }} & \frac{\Gamma, x: D \vdash p: \text { prop }}{\Gamma \vdash\{x: D \mid p\}: \text { dtype }}
\end{array}
$$

Proposition formation, $\Gamma \vdash p$ : prop

$$
\frac{\Gamma \vdash p_{1}, p_{2}: \text { prop }}{\Gamma \vdash p_{1} \wedge p_{2}: \text { prop }} \quad \frac{\Gamma \vdash i_{1}, i_{2}: \text { int }}{\Gamma \vdash i_{1} \leq i_{2}: \text { prop }} \quad \frac{\Gamma, x: \text { int } \vdash p: \text { prop }}{\Gamma \vdash \forall x . p: \text { prop }}
$$

Index term formation, $\Gamma \vdash i: D$

$$
\begin{gathered}
\frac{\Gamma: \text { context } x: D \in \Gamma}{\Gamma \vdash x: D} \quad \frac{\Gamma: \text { context }}{\Gamma \vdash n: \text { int }} \quad \frac{\Gamma: \text { context }}{\Gamma \vdash f: \text { float }} \\
\frac{\Gamma \vdash i: D \text { array }}{\Gamma \vdash \operatorname{len}(i): \text { int }} \quad \frac{\Gamma \vdash i_{1}: \text { int } \quad \Gamma \vdash i_{2}: \text { int }}{\Gamma \vdash i_{1}+i_{2}: \text { int }} \\
\frac{\Gamma \vdash i_{1}: D \quad \ldots \quad \Gamma \vdash i_{n}: D}{\Gamma \vdash\left[i_{1}, \ldots, i_{n}\right]: D \text { array }} \\
\frac{\Gamma \vdash i_{1}: D \text { array } \quad \Gamma \vdash 1 \leq i_{2} \leq \operatorname{len}\left(i_{1}\right) \text { true }}{\Gamma \vdash i_{1}\left[i_{2}\right]: D} \\
\frac{\Gamma \vdash i: D \quad \Gamma \vdash p\{i / x\} \text { true }}{\Gamma \vdash i:\{x: D \mid p\}} \quad \frac{\Gamma \vdash i: D_{1} \quad \Gamma \vdash D_{1}<: D_{2}}{\Gamma \vdash i: D_{2}}
\end{gathered}
$$

Context formation, $\Gamma:$ context

$$
\overline{\varepsilon: \text { context }} \quad \frac{\Gamma: \text { context } \Gamma \vdash D: \text { dtype } x \notin \Gamma, D}{\Gamma, x: D: \text { context }}
$$

\section{Figure 16. Formation rules}

Program reduction Figure 8 contains an excerpt of the reduction rules for programs. Figure 26 presents the program reduction rules.
Datatype subtyping, $\Gamma \vdash D<: D$

$$
\begin{gathered}
\frac{\Gamma: \text { context }}{\Gamma \vdash \text { int }<: \text { int }} \quad \frac{\Gamma: \text { context }}{\Gamma \vdash \text { float }<: \text { float }} \quad \frac{\Gamma \vdash D: \text { dtype }}{\Gamma \vdash D \text { array }<: D \text { array }} \\
\frac{\Gamma \vdash D_{1}<: D_{2} \quad \Gamma, x: D_{1} \vdash p \text { true }}{\Gamma \vdash D_{1}<:\left\{x: D_{2} \mid p\right\}} \\
\frac{\Gamma \vdash D_{1}<: D_{2} \quad \Gamma, x: D_{1} \vdash p: \text { prop }}{\Gamma \vdash\left\{x: D_{1} \mid p\right\}<: D_{2}}
\end{gathered}
$$

Datatype equality, $\Gamma \vdash D \equiv D$

$$
\frac{\Gamma \vdash D_{1}<: D_{2} \quad \Gamma \vdash D_{2}<: D_{1}}{\Gamma \vdash D_{1} \equiv D_{2}}
$$

Proposition entailment, $\Gamma \vdash p$ true

$$
\frac{\Gamma \vdash p: \text { prop } \quad \text { formulae }(\Gamma) \vDash p}{\Gamma \vdash p \text { true }}
$$

Formulae in a context, formulae $(\Gamma)$

$$
\begin{array}{ll}
\text { formulae }(\varepsilon) & \triangleq \emptyset \\
\text { formulae }(\Gamma, x: D) & \triangleq \operatorname{formulae}(\Gamma) \cup \operatorname{forms}(x: D) \\
\operatorname{forms}(x: \text { int }) & \triangleq \operatorname{forms}(x: \text { float }) \triangleq \operatorname{true} \\
\operatorname{forms}(h:\{x: D \mid p\}) & \triangleq \operatorname{forms}(x: D) \wedge p\{h / x\} \\
\operatorname{forms}(h: D \text { array }) & \triangleq \forall x .1 \leq x \leq \operatorname{len}(h) \rightarrow \operatorname{forms}(h[x]: D)
\end{array}
$$

Figure 17. Datatype subtyping and proposition entailment 
Type equality, $\Gamma \vdash T \equiv T$ :

$$
\frac{\Gamma: \text { context }}{\Gamma \vdash \text { skip } \equiv \text { skip }}
$$

$\Gamma \vdash i_{1}, i_{2}=i_{3}, i_{4}$ true $\quad \Gamma \vdash D_{1} \equiv D_{2}$

$\Gamma \nvdash i_{1}, i_{2} \neq$ rank true $\quad\left(\Gamma \vdash 1 \leq i_{1}, i_{2} \leq\right.$ size $\wedge i_{1} \neq i_{2}$ true $)$

$\Gamma \vdash$ message $i_{1} i_{2} D_{1} \equiv$ message $i_{3} i_{4} D_{2}$

$\Gamma \vdash i_{1}=i_{2}$ true $\quad \Gamma \vdash D_{1} \equiv D_{2}$

$\left(\Gamma \vdash 1 \leq i_{1} \leq\right.$ size true $) \quad\left(\Gamma \vdash D_{1}<: D_{3}\right.$ array $)$

$\Gamma \vdash$ scatter $i_{1} D_{1} \equiv$ scatter $i_{2} D_{2}$ see scatter

$\Gamma \vdash$ gather $i_{1} D_{1} \equiv$ gather $i_{2} D_{2}$

$\Gamma \vdash i_{1}=i_{2}$ true $\quad\left(\Gamma \vdash 1 \leq i_{1} \leq\right.$ size true $)$

$\Gamma \vdash$ reduce $i_{1} \equiv$ reduce $i_{2}$

$\frac{\Gamma \vdash D_{1} \equiv D_{2} \quad \Gamma, x: D_{1} \vdash T_{1} \equiv T_{2}}{\Gamma \vdash \operatorname{val} x: D_{1} \cdot T_{1} \equiv \operatorname{val} x: D_{2} \cdot T_{2}}$

$\Gamma \vdash i_{1}=i_{2}$ true $\quad \Gamma \vdash D_{1} \equiv D_{2} \quad \Gamma, x: D_{1} \vdash T_{1} \equiv T_{2}$

$\left(\Gamma \vdash 1 \leq i_{1} \leq\right.$ size true $)$

$\Gamma \vdash$ broadcast $i_{1} x: D_{1} \cdot T_{1} \equiv$ broadcast $i_{2} x: D_{2} \cdot T_{2}$

$$
\frac{\Gamma \vdash T_{1} \equiv T_{3} \quad \Gamma \vdash T_{2} \equiv T_{4}}{\Gamma \vdash T_{1} ; T_{2} \equiv T_{3} ; T_{4}}
$$

$\Gamma \vdash p_{1} \leftrightarrow p_{2}$ true $\quad \Gamma \vdash T_{1} \equiv T_{2}:$ type $\quad \Gamma \vdash T_{1}^{\prime} \equiv T_{2}^{\prime}:$ type

$\Gamma \vdash p_{1} ? T_{1}: T_{1}^{\prime} \equiv p_{2} ? T_{2}: T_{2}^{\prime}:$ type

$\Gamma \nvdash i_{1} \leq 1$ true $\quad \Gamma \nvdash i_{1}>1$ true $\quad \Gamma \vdash i_{1}=i_{2}$ true

$\Gamma, x:\left\{y:\right.$ int $\left.\mid y \leq i_{1}\right\} \vdash T_{1} \equiv T_{2}$

$\Gamma \vdash \forall x \leq i_{1} . T_{1} \equiv \forall x \leq i_{2} . T_{2}$

$\Gamma \vdash i \geq 1$ true $\quad(\Gamma, x:\{y:$ int $\mid y \leq i\} \vdash T:$ type $)$

$\Gamma \vdash \forall x \leq i . T \equiv(T\{i / x\} ; \forall x \leq i-1 . T)$

$\Gamma \vdash i<1$ true $\quad(\Gamma, x:\{y:$ int $\mid y \leq i\} \vdash T:$ type $)$

$\Gamma \vdash \forall x \leq i . T \equiv$ skip

$\Gamma \vdash i_{1}, i_{2} \neq$ rank true

$\left(\Gamma \vdash 1 \leq i_{1}, i_{2} \leq\right.$ size $\wedge i_{1} \neq i_{2}$ true $) \quad(\Gamma \vdash D:$ dtype $)$

$\Gamma \vdash$ message $i_{1} i_{2} D \equiv$ skip

$\frac{(\Gamma \vdash T: \text { type })}{\Gamma \vdash T ; \text { skip } \equiv T} \quad \frac{(\Gamma \vdash T: \text { type })}{\Gamma \vdash \text { skip } T \equiv T} \quad \frac{\left(\Gamma \vdash T_{1}, T_{2}, T_{3}: \text { type }\right)}{\Gamma \vdash\left(T_{1} ; T_{2}\right) ; T_{3} \equiv T_{1} ;\left(T_{2} ; T_{3}\right)}$

$\underline{\Gamma \vdash T_{1} \equiv T_{2}} \quad \frac{\Gamma \vdash T_{1} \equiv T_{2} \quad \Gamma \vdash T_{2} \equiv T_{3}}{\Gamma \vdash T_{2}}$

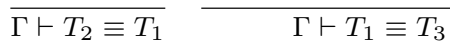

\section{Figure 18. Type equality}

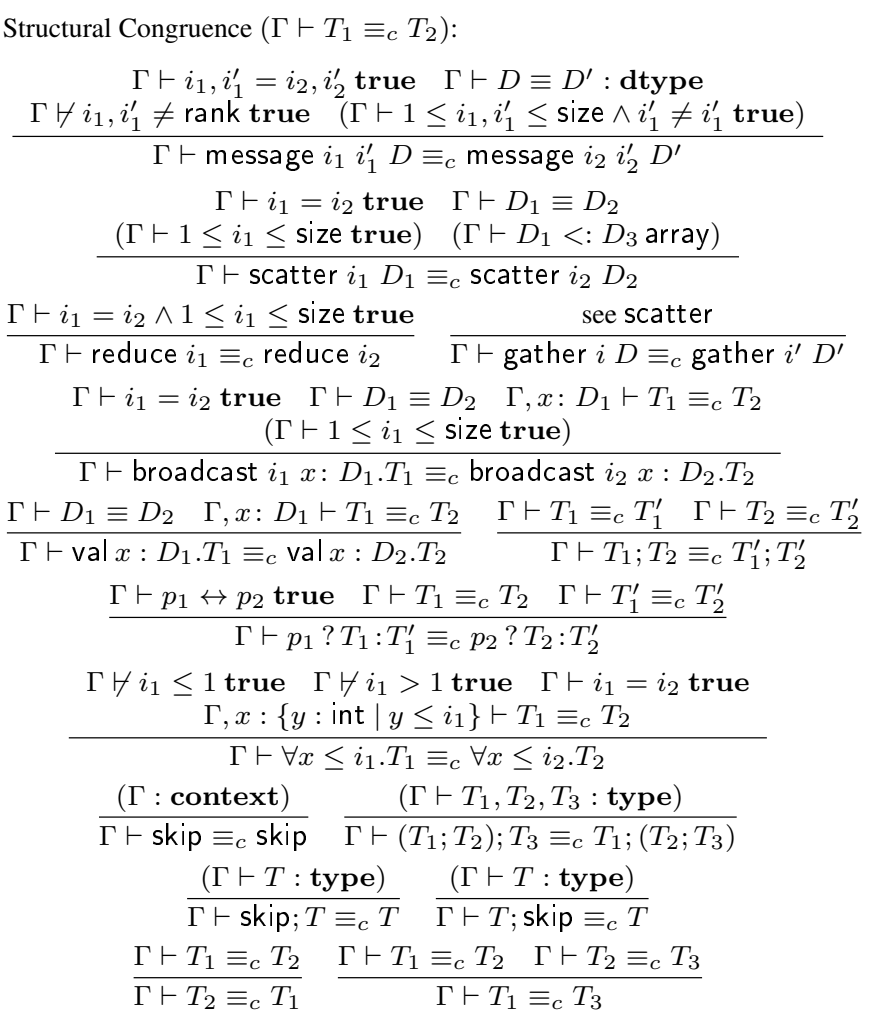

Figure 19. Structural congruence

Type Conversion $\left(\Gamma \vdash T_{1} \Rightarrow T_{2}\right)$

$$
\frac{(\Gamma \text { : context })}{\Gamma \vdash \text { skip } \Rightarrow \text { skip }} \quad \frac{\Gamma \vdash T_{1} \Rightarrow T_{1}^{\prime} \quad \Gamma \vdash T_{2} \Rightarrow T_{2}^{\prime}}{\Gamma \vdash T_{1} ; T_{2} \Rightarrow T_{1}^{\prime} ; T_{2}^{\prime}}
$$

$\Gamma \nvdash i_{1}, i_{2} \neq$ rank true $\quad\left(\left(\Gamma \vdash\right.\right.$ message $i_{1} i_{2} D:$ type $\left.)\right)$

$\Gamma \vdash$ message $i_{1} i_{2} D \Rightarrow$ message $i_{1} i_{2} D$

$(\Gamma \vdash 1 \leq i \leq$ size true $) \quad((\Gamma \vdash$ scatter $i D$ : type $))$

$\overline{\Gamma \vdash \text { reduce } i \Rightarrow \text { reduce } i} \quad \overline{\Gamma \vdash \text { scatter } i D \Rightarrow \text { scatter } i D}$

$((\Gamma \vdash$ gather $i D:$ type $)) \quad \Gamma, x: D \vdash T \Rightarrow T^{\prime}$

$\overline{\Gamma \vdash \text { gather } i D \Rightarrow \text { gather } i D} \quad \overline{\Gamma \vdash \operatorname{val} x: D . T \Rightarrow \operatorname{val} x: D . T^{\prime}}$

$\Gamma, x: D \vdash T \Rightarrow T^{\prime} \quad(\Gamma \vdash 1 \leq i \leq$ size true $)$

$\Gamma \vdash$ broadcast $i x: D . T \Rightarrow$ broadcast $i x: D . T^{\prime}$

$\Gamma \vdash T_{1} \Rightarrow T_{1}^{\prime} \quad \Gamma \vdash T_{2} \Rightarrow T_{2}^{\prime} \quad(\Gamma \vdash p:$ prop $)$

$\Gamma \vdash p ? T_{1}: T_{2} \Rightarrow p ? T_{1}^{\prime}: T_{2}^{\prime}$

$\Gamma \nvdash i \geq 1$ true $\quad \Gamma \nvdash i<1$ true $\quad \Gamma \vdash i<1$ true

$\frac{\left(\Gamma, x:\{y: \text { int } \mid y \leq i\} \vdash T \Rightarrow T^{\prime}\right)}{\Gamma \vdash \forall x \leq i . T \Rightarrow \forall x \leq i . T^{\prime}} \quad \frac{((\Gamma \vdash \forall x \leq i . T: \text { type }))}{\Gamma \vdash \forall x \leq i . T \Rightarrow \text { skip }}$

$\Gamma \vdash i \geq 1$ true $\Gamma \vdash T\{i / x\} \Rightarrow T^{\prime}$

$\Gamma \vdash \forall x \leq i-1 . T \Rightarrow T^{\prime \prime} \quad((\Gamma \vdash \forall x \leq i . T:$ type $))$

$\Gamma \vdash \forall x \leq i . T \Rightarrow T^{\prime} ; T^{\prime \prime}$

$\Gamma \vdash i_{1}, i_{2} \neq$ rank true $\quad\left(\left(\Gamma \vdash\right.\right.$ message $i_{1} i_{2} D:$ type $\left.)\right)$

$\Gamma \vdash$ message $i_{1} i_{2} D \Rightarrow$ skip

$((T))$ denotes the premises to the type formation rules for $T$ as in Fig. 16

Figure 20. Type conversion 
Datatype formation, $\Gamma \vdash D:$ dtype

$$
\frac{\Gamma \vdash D: \text { dtype }}{\Gamma \vdash D \text { ref : dtype }}
$$

Index formation, $\Gamma \vdash i: D$

$$
\begin{aligned}
& \frac{\Gamma \text { : context } r: D \in \Gamma}{\Gamma \vdash r: D} \quad \frac{\Gamma \vdash i: D}{\Gamma \vdash \operatorname{mkref} i: D \text { ref }} \quad \frac{\Gamma \vdash i: D \text { ref }}{\Gamma \vdash t i: D} \\
& \frac{\Gamma \vdash i_{1}: D \operatorname{ref} \quad \Gamma \vdash i_{2}: D}{\Gamma \vdash i_{1}:=i_{2}: D}
\end{aligned}
$$

Context formation, $\Gamma$ : context

$$
\frac{\Gamma: \text { context } \quad \Gamma \vdash D: \text { dtype } \quad r \notin \Gamma, D}{\Gamma, r: D: \text { context }}
$$

Datatype subtyping, $\Gamma \vdash D<: D$

$$
\frac{\Gamma \vdash D_{1} \equiv D_{2}}{\Gamma \vdash D_{1} \text { ref }<: D_{2} \text { ref }}
$$

\section{Figure 21. Reference formation}

Expression formation, $\Gamma \vdash e: T$ :

$$
\begin{aligned}
& \underline{(\Gamma \text { : context })} \quad \Gamma \vdash 1 \leq i_{1} \leq \text { size } \wedge i_{1} \neq \text { rank true } \quad \Gamma \vdash i_{2}: D
\end{aligned}
$$

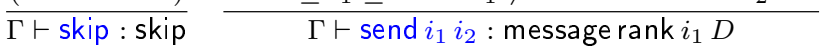

$$
\begin{aligned}
& \Gamma \vdash 1 \leq i_{1} \leq \text { size } \wedge i_{1} \neq \text { rank true } \Gamma \vdash i_{2}: D \text { ref } \\
& \Gamma \vdash \text { receive } i_{1} i_{2}: \text { message } i_{1} \text { rank } D \\
& \Gamma \vdash i: D \quad \Gamma, x: D \vdash e: T \\
& \overline{\Gamma \vdash \text { let } x: D=\operatorname{val} i \text { in } e: \operatorname{val} x: D . T} \\
& \Gamma \vdash 1 \leq i_{1} \leq \text { size true } \Gamma \vdash i_{2}: \text { float } \quad \Gamma \vdash i_{3}: \text { float ref } \\
& \Gamma \vdash \text { reduce } i_{1} i_{2} i_{3}: \text { reduce } i_{1} \\
& \Gamma \vdash 1 \leq i_{1} \leq \text { size true } \quad \Gamma \vdash i_{2} \text { : float array ref } \\
& \Gamma,\left\{\text { rank }=i_{1}\right\} \vdash i_{3}:\left\{x \text { : float array } \mid \text { len }(x)=\operatorname{size} * \operatorname{len}\left(i_{2}\right)\right\} \\
& \Gamma \vdash \text { let } x: D=\text { broadcast } i_{1} i_{2} \text { in } e \text { : broadcast } i_{1} x: D . T \\
& \frac{\Gamma, p \vdash e_{1}: T_{1} \quad \Gamma, \neg p \vdash e_{2}: T_{2} \quad \text { rank } \notin \mathrm{fv}(p)}{\Gamma \vdash \text { ifc } p \text { then } e_{1} \text { else } e_{2}: p ? T_{1}: T_{2}} \\
& \frac{\Gamma \vdash e_{1}: T_{1} \quad \Gamma \vdash e_{2}: T_{2}}{\Gamma \vdash e_{1} ; e_{2}: T_{1} ; T_{2}} \quad \frac{\Gamma, x:\{y: \text { int } \mid y \leq i\} \vdash e: T}{\Gamma \vdash \text { for } x: i . .1 \mathrm{do} e: \forall x \leq i . T} \\
& \frac{\Gamma, p \vdash e_{1}: T \quad \Gamma, \neg p \vdash e_{2}: T}{\Gamma \vdash \text { if } p \text { then } e_{1} \text { else } e_{2}: T} \\
& \frac{\Gamma, p \vdash e: \text { skip }}{\Gamma \vdash \text { while } p \text { do } e \text { :skip }} \quad \frac{\Gamma \vdash i: D \quad \Gamma, x: D \vdash e: T \quad x \notin \mathrm{fv}(T)}{\Gamma \vdash \operatorname{let} x: D=i \text { in } e: T} \\
& \frac{\Gamma \vdash e: T_{1} \quad \Gamma \vdash T_{1} \equiv T_{2}: \text { type }}{\Gamma \vdash e: T_{2}}
\end{aligned}
$$

In all rules, $T$ and $D$ contain no ref datatypes.

Figure 22. Expression formation
Stores, $\rho:$ store

$$
\varepsilon: \text { store } \frac{\rho: \text { store } r \notin \rho \quad \rho \text { to } \Gamma \quad \Gamma \vdash v: D}{\rho, r:=v: \text { store }}
$$

Stores as contexts, $\rho$ to $\Gamma$

$$
\begin{gathered}
(\varepsilon: \text { store }) \text { to }(\varepsilon: \text { context }) \\
\frac{\rho \text { to } \Gamma \quad \Gamma \vdash v: D \quad(r \notin \rho, \Gamma, D)}{(\rho, r:=v) \text { to }(\Gamma, r: D \text { ref })}
\end{gathered}
$$

Figure 23. Store formation and store-to-context conversion

Index term evaluation, $\left(\rho_{1}, i\right) \downarrow^{n, k}\left(\rho_{2}, v\right): D$ :

$$
\begin{aligned}
& \frac{(\rho: \text { store })}{(\rho, m) \downarrow^{n, k}(\rho, m): \text { int }} \quad \frac{(\rho: \text { store })}{(\rho, f) \downarrow^{n, k}(\rho, f): \text { float }} \\
& \frac{(\rho: \text { store })}{(\rho, \text { size }) \downarrow^{n, k}(\rho, n) \text { : int }} \quad \frac{(\rho: \text { store })}{(\rho, \text { rank }) \downarrow^{n, k}(\rho, k): \text { int }} \\
& \frac{r:=v \in \rho \quad \rho \vdash v: D \quad(\rho: \text { store })}{(\rho, r) \downarrow^{n, k}(\rho, r): D \text { ref }} \\
& \left(\rho_{1}, i\right) \downarrow^{n, k}\left(\rho_{2}, v\right): D \quad r \notin \rho_{2} \\
& \overline{\left(\rho_{1}, \text { mkref } i\right) \downarrow^{n, k}\left(\left(\rho_{2}, r:=v\right), r\right): D \text { ref }} \\
& \frac{\left(\rho_{1}, i\right) \downarrow^{n, k}\left(\rho_{2}, r\right): D \text { ref } \quad r:=v \in \rho_{2}}{\left(\rho_{1}, ! i\right) \downarrow^{n, k}\left(\rho_{2}, v\right): D} \\
& \frac{\left(\rho_{1}, i_{1}\right) \downarrow^{n, k}\left(\rho_{2}, r\right): D \operatorname{ref} \quad\left(\rho_{2}, i_{2}\right) \downarrow^{n, k}\left(\rho_{3}, v\right): D}{\left(\rho_{1}, i_{1}:=i_{2}\right) \downarrow^{n, k}\left(\rho_{3}[r:=v], v\right): D} \\
& \frac{\left(\rho_{1}, i_{1}\right) \downarrow^{n, k}\left(\rho_{2}, v_{1}\right): \text { int } \quad\left(\rho_{2}, i_{2}\right) \downarrow^{n, k}\left(\rho_{3}, v_{2}\right): \text { int }}{\left(\rho_{1}, i_{1}+i_{2}\right) \downarrow^{n, k}\left(\rho_{3}, v_{1}+v_{2}\right): \text { int }} \\
& \frac{\left(\rho_{1}, i_{1}\right) \downarrow^{n, k}\left(\rho_{2}, v_{1}\right): D \quad \ldots \quad\left(\rho_{n}, i_{n}\right) \downarrow^{n, k}\left(\rho_{n+1}, v_{n}\right): D}{\left(\rho_{1},\left[i_{1}, \ldots, i_{n}\right]\right) \downarrow^{n, k}\left(\rho_{n+1},\left[v_{1}, \ldots, v_{n}\right]\right): D \text { array }} \\
& \left(\rho_{1}, i_{1}\right) \downarrow^{n, k}\left(\rho_{2},\left[v_{1}, \ldots, v_{l}\right]\right):\{x: D \text { array } \mid \operatorname{len}(x)=l\} \\
& \left(\rho_{2}, i_{2}\right) \downarrow^{n, k}\left(\rho_{3}, m\right):\{y \text { : int } \mid 1 \leq y \leq l\} \\
& \underline{\left(\rho_{1}, i\right) \downarrow^{n, k}\left(\rho_{2},\left[v_{1}, \ldots, v_{n}\right]\right): D \text { array }} \\
& \left(\rho_{1}, \operatorname{len}(i)\right) \downarrow^{n, k}\left(\rho_{2}, n\right) \text { : int } \\
& \frac{\left(\rho_{1}, i\right) \downarrow^{n, k}\left(\rho_{2}, v\right): D_{1} \quad \rho_{2} \vdash D_{2}<: D_{1}}{\left(\rho_{1}, i\right) \downarrow^{n, k}\left(\rho_{2}, v\right): D_{2}} \\
& \frac{\left(\rho_{1}, i\right) \downarrow^{n, k}\left(\rho_{2}, v\right): D \quad \Gamma^{n, k}, \rho_{2} \vdash p\{i / x\} \text { true }}{\left(\rho_{1}, i\right) \downarrow^{n, k}\left(\rho_{2}, v\right):\{x: D \mid p\}}
\end{aligned}
$$

Figure 24. Index term evaluation 
Process reduction, $p \rightarrow^{n, k} q$ :

$$
\begin{aligned}
& \frac{(\rho, i) \downarrow^{n, k}\left(\rho^{\prime}, v\right): D \quad\left(\Gamma^{n, k}, \rho, x: D \vdash e: T\right) \quad(x \notin \mathrm{fv}(T))}{(\rho, \text { let } x: D=i \text { in } e) \rightarrow^{n, k}\left(\rho^{\prime}, e\{v / x\}\right)} \quad \frac{\Gamma^{n, k}, \rho \vdash p \operatorname{true} \quad\left(\Gamma^{n, k}, p, \rho \vdash e_{1}: T\right) \quad\left(\Gamma^{n, k}, \neg p, \rho \vdash e_{2}: T\right)}{\left(\rho, \text { if } p \text { then } e_{1} \text { else } e_{2}\right) \rightarrow^{n, k}\left(\rho, e_{1}\right)} \\
& \underline{\Gamma^{n, k}, \rho \vdash \neg p \text { true } \quad\left(\Gamma^{n, k}, \neg p, \rho \vdash e_{1}: T\right) \quad\left(\Gamma^{n, k}, p, \rho \vdash e_{2}: T\right)} \quad \Gamma^{n, k}, \rho \vdash p \text { true } \quad\left(\Gamma^{n, k}, p, \rho \vdash e: \text { skip }\right) \\
& \left(\rho, \text { if } p \text { then } e_{1} \text { else } e_{2}\right) \rightarrow^{n, k}\left(\rho, e_{2}\right) \quad \frac{\left.T^{\prime} \text { while } p \text { do } e\right) \rightarrow^{n, k}(\rho,(e ; \text { while } p \text { do } e))}{(\rho, n)} \\
& \frac{\left(\Gamma^{n, k}, \rho \vdash e: T\right)}{(\rho,(\text { skip } ; e)) \rightarrow^{n, k}(\rho, e)} \quad \frac{\Gamma^{n, k}, \rho \vdash \neg p \text { true } \quad\left(\Gamma^{n, k}, \neg p, \rho \vdash e: \text { skip }\right)}{(\rho, \text { while } p \text { do } e) \rightarrow^{n, k}(\rho, \text { skip })} \\
& \frac{\Gamma^{n, k}, \rho \vdash i \geq 1 \text { true } \quad\left(\Gamma^{n, k}, \rho, x:\{y: \text { int } \mid y \leq i\} \vdash e: T\right)}{(\rho, \text { for } x: i . .1 \text { do } e) \rightarrow^{n, k}(\rho,(e\{i / x\} ; \text { for } x: i-1 . .1 \text { do } e))} \quad \frac{\Gamma^{n, k}, \rho \vdash i<1 \text { true } \quad\left(\Gamma^{n, k}, \rho, x:\{y: \text { int } \mid y \leq i\} \vdash e: T\right)}{(\rho, \text { for } x: i . .1 \text { do } e) \rightarrow^{n, k}(\rho, \text { skip })} \\
& \frac{\left(\rho, e_{1}\right) \rightarrow^{n, k}\left(\rho^{\prime}, e_{3}\right) \quad\left(\Gamma^{n, k}, \rho \vdash e_{2}: T\right)}{\left(\rho,\left(e_{1} ; e_{2}\right)\right) \rightarrow^{n, k}\left(\rho^{\prime},\left(e_{3} ; e_{2}\right)\right)}
\end{aligned}
$$

Program reduction, $P_{1} \rightarrow P_{2}$ :

\section{Figure 25. Process reduction}

$$
\begin{aligned}
& i_{l} \downarrow^{n} m \quad\left(\rho_{l}, i_{l}^{\prime}\right) \downarrow^{n, l}\left(\rho_{l}^{\prime}, v\right): D \quad i_{m} \downarrow^{n} l \quad\left(\rho_{m}, i_{m}^{\prime}\right) \downarrow^{n, m}\left(\rho_{m}^{\prime}, r\right): D \text { ref } \quad(l \neq m) \\
& \left(\Gamma^{n, l} \vdash e_{l}: T\right) \quad\left(\Gamma^{n, m} \vdash e_{m}: T\right) \quad\left(\Gamma^{n, k} \vdash q_{k}: T\right) \quad(k=1 . . n, k \neq l, m) \\
& q_{1}, \ldots, q_{l-1},\left(\rho_{l}, \text { send } i_{l} i_{l}^{\prime} ; e_{l}\right), q_{l+1}, \ldots, q_{m-1},\left(\rho_{m}, \text { receive } i_{m} i_{m}^{\prime} ; e_{m}\right), q_{m+1} \ldots, q_{n} \rightarrow \\
& q_{1}, \ldots, q_{l-1},\left(\rho_{l}^{\prime}, e_{l}\right), q_{l+1}, \ldots, q_{m-1},\left(\rho_{m}^{\prime}[r:=v], e_{m}\right), q_{m+1} \ldots, q_{n} \\
& i_{k} \downarrow^{n} l \quad\left(\rho_{k}, i_{k}^{\prime}\right) \downarrow^{n, k}\left(\rho_{k}^{\prime}, f_{k}\right) \text { : float } \quad\left(\rho_{l}^{\prime}, i_{l}^{\prime \prime}\right) \downarrow^{n, l}\left(\rho_{l}^{\prime \prime}, r\right) \text { : float ref } \quad(k=1 . . n) \\
& \overline{\left(\rho_{k}, \text { reduce } i_{k} i_{k}^{\prime} i_{k}^{\prime \prime}\right)_{k=1}^{n} \rightarrow\left(\rho_{k}^{\prime}, \text { skip }\right)_{k=1}^{l-1},\left(\rho_{l}^{\prime \prime}\left[r:=\max \left(v_{1}, . ., v_{n}\right)\right], \text { skip }\right),\left(\rho_{k}^{\prime}, \text { skip }\right)_{k=l+1}^{n}} \\
& i_{k} \downarrow^{n} l \quad\left(\rho_{k}, i_{k}^{\prime}\right) \downarrow^{n, k}\left(\rho_{k}^{\prime}, r_{k}\right): \text { float array ref }\left(\rho_{l}^{\prime}, i_{l}^{\prime \prime}\right) \downarrow^{n, l}\left(\rho_{l}^{\prime \prime},\left[\vec{v}_{1}, \ldots, \vec{v}_{n}\right]\right):\left\{x: \text { float array } \mid \text { len }(x)=\operatorname{size} * \operatorname{len}\left(i_{k}^{\prime}\right)\right\} \\
& \left(\Gamma^{n, k}, \rho_{k},\left\{\text { rank }=i_{k}\right\} \vdash i_{k}^{\prime \prime}:\left\{x: \text { float array } \mid \operatorname{len}(x)=\operatorname{size} * \operatorname{len}\left(i_{k}^{\prime}\right)\right\}\right) \quad(k=1 . . n) \\
& \left(\rho_{k}, \text { scatter } i_{k} i_{k}^{\prime} i_{k}^{\prime \prime}\right)_{k=1}^{n} \rightarrow\left(\rho_{k}^{\prime}\left[r_{k}:=\left[\vec{v}_{k}\right]\right], \text { skip }\right)_{k=1}^{l-1},\left(\rho_{l}^{\prime \prime}\left[r_{l}:=\left[\vec{v}_{l}\right]\right], \text { skip }\right),\left(\rho_{k}^{\prime}\left[r_{k}:=\left[\vec{v}_{k}\right]\right], \text { skip }\right)_{k=l+1}^{n} \\
& i_{k} \downarrow^{n} l \quad\left(\rho_{k}, i_{k}^{\prime}\right) \downarrow^{n, k}\left(\rho_{k}^{\prime},\left[\vec{v}_{k}\right]\right): \text { float array }\left(\rho_{l}^{\prime}, i_{l}^{\prime \prime}\right) \downarrow^{n, l}\left(\rho_{l}^{\prime \prime}, r\right):\left\{x: \text { float array } \mid \text { len }(x)=\operatorname{size} * \text { len }\left(i_{k}^{\prime}\right)\right\} \text { ref } \\
& \left(\Gamma^{n, k}, \rho_{k},\left\{\text { rank }=i_{k}\right\} \vdash i_{k}^{\prime \prime}:\left\{x \text { : float array } \mid \text { len }(x)=\operatorname{size} * \operatorname{len}\left(i_{k}^{\prime}\right)\right\} \text { ref }\right) \quad(k=1 . . n) \\
& \left(\rho_{k}, \text { gather } i_{k} i_{k}^{\prime} i_{k}^{\prime \prime}\right)_{k=1}^{n} \rightarrow\left(\rho_{k}^{\prime}, \text { skip }\right)_{k=1}^{l-1},\left(\rho_{l}^{\prime \prime}\left[r:=\left[\vec{v}_{1}, \ldots, \vec{v}_{n}\right]\right], \text { skip }\right),\left(\rho_{k}^{\prime}, \text { skip }\right)_{k=l+1}^{n} \\
& \frac{i_{k} \downarrow^{n} l \quad\left(\rho_{l}, i_{l}^{\prime}\right) \downarrow^{n, l}\left(\rho_{l}^{\prime}, v\right): D \quad\left(\Gamma^{n} \vdash 1 \leq i_{k} \leq n \text { true }\right) \quad\left(\Gamma^{n, k}, \rho_{k} \vdash i_{k}^{\prime}: D\right) \quad\left(\Gamma^{n, k}, x: D, \rho_{k} \vdash e_{k}: T\right) \quad(k=1 \ldots n)}{\left(\rho_{k}, \text { let } x: D=\text { broadcast } i_{k} i_{k}^{\prime} \text { in } e_{k}\right)_{k=1}^{n} \rightarrow\left(\rho_{k}, e_{k}\{v / x\}\right)_{k=1}^{l-1},\left(\rho_{l}^{\prime}, e_{l}\{v / x\}\right),\left(\rho_{k}, e_{k}\{v / x\}\right)_{k=l+1}^{n}} \\
& \frac{i_{k} \downarrow^{n} v \quad\left(\Gamma^{n, k}, x: D, \rho_{k} \vdash e_{k}: T\right) \quad(k=1 . . n)}{\left(\rho_{k}, \text { let } x: D=\operatorname{val} i_{k} \text { in } e_{k}\right)_{k=1}^{n} \rightarrow\left(\rho_{k}, e_{k}\{v / x\}\right)_{k=1}^{n}} \\
& \Gamma^{n} \vdash p_{k} \text { true } \quad\left(\Gamma^{n, k}, \rho_{k} \vdash e_{k}: T\right) \quad\left(\Gamma^{n, k}, \rho_{k} \vdash e_{k}^{\prime}: T^{\prime}\right) \\
& \overline{\left(\rho_{1} \text {, ifc } p_{1} \text { then } e_{1} \text { else } e_{1}^{\prime}\right), \ldots,\left(\rho_{n}, \text { ifc } p_{n} \text { then } e_{n} \text { else } e_{n}^{\prime}\right) \rightarrow\left(\rho_{1}, e_{1}\right), \ldots\left(\rho_{n}, e_{n}\right)} \\
& \frac{\left(q_{k}, e_{k}\right)_{k=1}^{n} \rightarrow\left(q_{k}^{\prime}, e_{k}^{\prime \prime}\right)_{k=1}^{n}\left(\Gamma^{n, k} \vdash e_{k}^{\prime}: T_{k}\right) \quad\left(T_{1}, \ldots, T_{n}: \text { ptype }\right) \quad(k=1 . . n)}{\left(q_{k},\left(e_{k} ; e_{k}^{\prime}\right)\right)_{k=1}^{n} \rightarrow\left(q_{k}^{\prime},\left(e_{k}^{\prime \prime} ; e_{k}^{\prime}\right)\right)_{k=1}^{n}} \\
& \frac{q_{l} \rightarrow^{n, l} q_{l}^{\prime}\left(\Gamma^{n, k} \vdash q_{k}: T_{k}\right) \quad\left(T_{1}, \ldots, T_{n}: \text { ptype }\right) \quad(k=1 . . n)}{q_{1}, \ldots, q_{n} \rightarrow q_{1}, \ldots, q_{l-1}, q_{l}^{\prime}, q_{l+1}, \ldots, q_{n}}
\end{aligned}
$$

In all rules, $D$ and $T$ contain no ref types and rank $\notin \mathrm{fv}(D, T)$

Omitting dual rule for receive-send, the $\Gamma \vdash \neg p_{k}$ true rule for ifc, and integer/multidimensional arrays in scatter and gather rules.

Figure 26. Program reduction 


\section{Proofs}

This section includes the proofs for all theorems stated in the paper. For ease of navigation, we divide the section into proofs related to term types ( $\S \mathrm{D} .1)$, type equality including decidability ( $\S \mathrm{D} .2)$, program types $(\S \mathrm{D} .3)$, references $(\S \mathrm{D} .4)$, expressions (§ D.5), stores (§ D.6), processes (§ D.7), and programs (§ D.8).

\section{D.1 Results related to term types}

Main results in this section: agreement for type formation (Lemma D.1), weakening (Lemma D.2), strengthening (Lemma D.3), context exchange (Lemma D.8) $<$ : is a preorder (Lemma D.6), and the substitution lemma for types (Lemma D.7).

Lemma D.1 (agreement for type formation).

$$
\begin{array}{cccc}
\frac{\Gamma \vdash T: \text { type }}{\Gamma: \text { context }} & \frac{\Gamma \vdash D: \text { dtype }}{\Gamma: \text { context }} & \multicolumn{1}{c}{\frac{\Gamma \vdash p: \text { prop }}{\Gamma: \text { context }}} \\
\frac{\Gamma \vdash i: D}{\Gamma \vdash D: \text { dtype }} & \frac{\Gamma \vdash D_{1}<: D_{2}}{\Gamma \vdash D_{1}: \text { dtype } \Gamma \vdash D_{2}: \text { dtype }} & \frac{\Gamma \vdash p \text { true }}{\Gamma: \text { context }}
\end{array}
$$

Proof. By simultaneous rule induction on the various hypothesis.

Lemma D.2 (weakening). Let $\Gamma \vdash D:$ dtype. $^{3}$

$$
\begin{array}{cccc}
\frac{\Gamma \vdash T: \text { type }}{\Gamma, x: D \vdash T: \text { type }} & \frac{\Gamma \vdash D_{2}: \text { dtype }}{\Gamma, x: D \vdash D_{2}: \text { dtype }} & \frac{\Gamma \vdash p: \text { prop }}{\Gamma, x: D \vdash p: \text { prop }} \\
\frac{\Gamma \vdash i: D_{1}}{\Gamma, x: D \vdash i: D_{1}} & \frac{\Gamma \vdash D_{1}<: D_{2}}{\Gamma, x: D \vdash D_{1}<: D_{2}} & \frac{\Gamma \vdash p \text { true }}{\Gamma, x: D \vdash p \text { true }}
\end{array}
$$

Proof. By simultaneous rule induction on the various hypotheses.

Lemma D.3 (strengthening).

$$
\begin{array}{cl}
\frac{\Gamma, x: D \vdash T: \text { type } \quad x \notin \mathrm{fv}(T)}{\Gamma \vdash T: \text { type }} & \frac{\Gamma, x: D \vdash D_{1}: \text { dtype } \quad x \notin \mathrm{fv}\left(D_{1}\right)}{\Gamma \vdash D_{1}: \operatorname{dtype}} \\
\frac{\Gamma, x: D \vdash p: \text { prop } \quad x \notin \mathrm{fv}(p)}{\Gamma \vdash p: \text { prop }} & \frac{\Gamma, x: D \vdash i: D_{1} \quad x \notin \mathrm{fv}\left(D_{1}\right)}{\Gamma \vdash i: D_{1}} \\
\frac{\Gamma, x: D \vdash D_{1}<: D_{2} \quad x \notin \mathrm{fv}\left(D_{1}, D_{2}\right)}{\Gamma \vdash D_{1}<: D_{2}} & \frac{\Gamma, x: D \vdash p \operatorname{true} \quad x \notin \mathrm{fv}(p)}{\Gamma \vdash p \text { true }} \\
\frac{y: D_{1} \in \Gamma, x: D_{2}}{y: D_{1} \in \Gamma} & x \notin y, \mathrm{fv}\left(D_{1}\right)
\end{array}
$$

Proof. By simultaneous rule induction on the first hypothesis for each justified inference.

Lemma D.4 (inversion for subtyping).

1. If $\Gamma \vdash$ int $<: D$ then $D$ is int or $D$ is $\left\{x: D^{\prime} \mid p\right\}$ and $\Gamma \vdash D^{\prime}<$ : int.

2. If $\Gamma \vdash$ float $<: D$ then $D$ is float or $D$ is $\left\{x: D^{\prime} \mid p\right\}$ and $\Gamma \vdash D^{\prime}<:$ float.

3. If $\Gamma \vdash D_{1}$ array $<: D_{2}$ then $D_{2}$ is $D_{3}$ array and $\Gamma \vdash D_{1}<: D_{3}$ or $D_{2}$ is $\left\{x: D_{3} \mid p\right\}$ and $\Gamma \vdash D_{1}$ array $<: D_{3}$ and $\Gamma, x: D_{1}$ array $\vdash p$ true.

4. If $\Gamma \vdash\left\{x: D_{1} \mid p_{1}\right\}<: D_{2}$ then $\Gamma \vdash D_{1}<: D_{2}$ and $\Gamma, x: D_{1} \vdash p_{1}: \operatorname{prop}$ or $D_{2}$ is $\left\{y: D_{3} \mid p_{2}\right\}$ and $\Gamma \vdash\left\{x: D_{1} \mid p_{1}\right\}<: D_{3}$ and $\Gamma, y:\left\{x: D_{1} \mid p_{1}\right\} \vdash p_{2}$ true.

Proof. By a case analysis on the rules for subtyping.

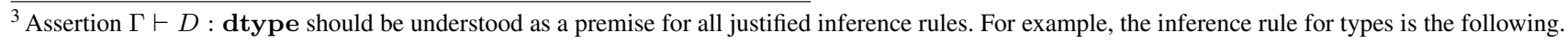

$$
\frac{\Gamma \vdash D: \text { dtype } \quad \Gamma \vdash T: \text { type }}{\Gamma, x: D \vdash T: \text { type }}
$$
}


Lemma D.5 (context subsumption). Let $\Gamma \vdash D_{2}<: D_{1}$.

$$
\begin{array}{ccc}
\frac{\Gamma, x: D_{1}: \text { context }}{\Gamma, x: D_{2}: \text { context }} & \frac{\Gamma, x: D_{1} \vdash T: \text { type }}{\Gamma, x: D_{2} \vdash T: \text { type }} & \frac{\Gamma, x: D_{1} \vdash D_{3}: \text { dtype }}{\Gamma, x: D_{2} \vdash D_{3}: \text { dtype }} \\
\frac{\Gamma, x: D_{1} \vdash p \text { true }}{\Gamma, x: D_{2} \vdash p \text { true }} & \frac{\Gamma, x: D_{1} \vdash p: \text { prop }}{\Gamma, x: D_{2} \vdash p: \text { prop }} & \frac{\Gamma, x: D_{1} \vdash i: D_{3}}{\Gamma, x: D_{2} \vdash i: D_{3}}
\end{array}
$$

Proof. By simultaneous rule induction on the various hypotheses.

Lemma D.6 ( $<$ : is a pre-order).

$$
\overline{\Gamma \vdash D<: D} \quad \frac{\Gamma \vdash D_{1}<: D_{2} \quad \Gamma \vdash D_{2}<: D_{3}}{\Gamma \vdash D_{1}<: D_{3}}
$$

sketch. Reflexivity follows by case analysis on assertion $\Gamma \vdash D:$ dtype. Transitivity is proved by induction on assertion $\Gamma \vdash D_{1}$ : dtype followed by induction on assertion $\Gamma \vdash D_{2}: \mathbf{d t y p e}$ using the inversion lemma for subtyping (Lemma D.4) and context subsumption (Lemma D.5).

Lemma D.7 (substitution lemma).

$$
\begin{aligned}
& \frac{\Gamma_{1}, x: D, \Gamma_{2} \vdash T: \text { type } \quad \Gamma_{1} \vdash i: D}{\Gamma_{1}, \Gamma_{2}\{i / x\} \vdash T\{i / x\}: \text { type }} \quad \frac{\Gamma_{1}, x: D, \Gamma_{2} \vdash D_{1}: \text { dtype } \quad \Gamma_{1} \vdash i: D}{\Gamma_{1}, \Gamma_{2}\{i / x\} \vdash D_{1}\{i / x\}: \text { dtype }} \\
& \frac{\Gamma_{1}, x: D, \Gamma_{2} \vdash p: \text { prop } \quad \Gamma_{1} \vdash i: D}{\Gamma_{1}, \Gamma_{2}\{i / x\} \vdash p\{i / x\}: \text { prop }} \quad \frac{\Gamma_{1}, x: D, \Gamma_{2} \vdash p \text { true } \quad \Gamma_{1} \vdash i: D}{\Gamma_{1}, \Gamma_{2}\{i / x\} \vdash p\{i / x\} \text { true }} \\
& \frac{\Gamma_{1}, x: D, \Gamma_{2} \vdash i_{1}: D_{1} \quad \Gamma_{1} \vdash i: D}{\Gamma_{1}, \Gamma_{2}\{i / x\} \vdash i_{1}\{i / x\}: D_{1}\{i / x\}} \quad \frac{\Gamma_{1}, x: D, \Gamma_{2} \vdash D_{1}<: D_{2} \quad \Gamma_{1} \vdash i: D}{\Gamma_{1}, \Gamma_{2}\{i / x\} \vdash D_{1}\{i / x\}<: D_{2}\{i / x\}} \\
& \frac{\Gamma_{1}, x: D, \Gamma_{2}: \text { context } \quad \Gamma_{1} \vdash i: D}{\Gamma_{1}, \Gamma_{2}\{i / x\}: \text { context }} \quad \frac{y: D \in\left(\Gamma_{1}, x: D, \Gamma_{2}\right) \quad \Gamma_{1} \vdash i: D}{y: D\{i / x\} \in \Gamma\{i / x\}}
\end{aligned}
$$

Proof. By simultaneous rule induction on the first hypothesis of each justified inference rule. We highlight a couple of cases.

$$
\text { hypothesis } \quad \Gamma_{1} \vdash i: D
$$

Case the derivation ends with $\Gamma_{1}, x: D, \Gamma_{2} \vdash$ broadcast $i_{1} y: D_{1} . T:$ type.

$$
\begin{array}{ll}
\text { rule premise } & \Gamma_{1}, x: D, \Gamma_{2} \vdash 1 \leq i_{1} \leq \text { size true } \\
\text { rule premise } & \Gamma_{1}, x: D, \Gamma_{2}, y: D_{1} \vdash T: \text { type } \\
1 \text {, induction, def. subs. } & \Gamma_{1}, \Gamma_{2}\{i / x\} \vdash 1 \leq i_{1}\{i / x\} \leq \text { size true } \\
2, \text { induction, def. subs. } & \Gamma_{1}, \Gamma_{2}\{i / x\}, y: D_{1}\{i / x\} \vdash T\{i / x\}: \text { type } \\
4,5, \text { formation, def. subs. } & \Gamma_{1}, \Gamma_{2}\{i / x\} \vdash\left(\text { broadcast } i_{1} y: D_{1} \cdot T\right)\{i / x\}: \text { type }
\end{array}
$$

Case the derivation ends with $\Gamma_{1}, x: D, \Gamma_{2} \vdash y: D_{1}$.

$$
\begin{array}{ll}
\text { rule premise } & \Gamma_{1}, x: D, \Gamma_{2}: \text { context } \\
\text { rule premise } & y: D_{1} \in\left(\Gamma_{1}, x: D, \Gamma_{2}\right)
\end{array}
$$

Subcase $y=x$

$$
\begin{array}{ll}
3, y=x & D_{1} \text { is } D \\
2, \text { context formation } & x \notin D \\
1,4,5, y=x, \text { def. subs. } & \Gamma_{1} \vdash y\{i / x\}: D_{1}\{i / x\} \\
6,2, \text { lemma D.2 } & \Gamma_{1}, \Gamma_{2}\{i / x\} \vdash y\{i / x\}: D_{1}\{i / x\}
\end{array}
$$

Subcase $y \neq x$

$$
1,2 \text {, induction } \quad \Gamma_{1}, \Gamma_{2}\{i / x\}: \text { context }
$$




$$
\begin{array}{ll}
1,3, \text { induction } & y: D_{1}\{i / x\} \in\left(\Gamma_{1}, \Gamma_{2}\{i / x\}\right) \\
8,9, \text { type formation } & \Gamma_{1}, \Gamma_{2}\{i / x\} \vdash y\{i / x\}: D_{1}\{i / x\}
\end{array}
$$

Case the derivation ends with $\Gamma_{1}, x: D, \Gamma_{2} \vdash i_{1}:\left\{y: D_{1} \mid p\right\}$.

$$
\begin{array}{ll}
\text { rule premise } & \Gamma_{1}, x: D, \Gamma_{2} \vdash i_{1}: D_{1} \\
\text { rule premise } & \Gamma_{1}, x: D, \Gamma_{2} \vdash p\left\{i_{1} / y\right\} \text { true } \\
2, \text { induction } & \Gamma_{1}, \Gamma_{2}\{i / x\} \vdash i_{1}\{i / x\}: D_{1}\{i / x\} \\
2, \text { induction } & \Gamma_{1}, \Gamma_{2}\{i / x\} \vdash p\left\{i_{1} / y\right\}\{i / x\} \text { true } \\
5,(y \neq x), \text { def. subs. } & \Gamma_{1}, \Gamma_{2}\{i / x\} \vdash p\{i / x\}\left\{i_{1} / y\right\} \text { true } \\
4,5, \text { index formation } & \Gamma_{1}, \Gamma_{2}\{i / x\} \vdash i_{1}\{i / x\}:\left\{y: D_{1}\{i / x\} \mid p\{i / x\}\right\} \\
7, \text { def. subs. } & \Gamma_{1}, \Gamma_{2}\{i / x\} \vdash i_{1}\{i / x\}:\left\{y: D_{1} \mid p\right\}\{i / x\}
\end{array}
$$

Case the derivation ends with $\Gamma_{1}, x: D, \Gamma_{2} \vdash p$ true.

$$
\begin{aligned}
& \text { rule premise } \\
& \text { rule premise } \\
& 2, \text { induction } \\
& 1,3 \text {, assumption on } \vDash \\
& 4,5, \text { true formation }
\end{aligned}
$$$$
\Gamma_{1}, x: D, \Gamma_{2} \vdash p: \text { prop }
$$$$
\text { formulae }\left(\Gamma_{1}, x: D, \Gamma_{2}\right) \vDash p
$$$$
\Gamma_{1}, \Gamma_{2}\{i / x\} \vdash p\{i / x\} \text { : prop }
$$$$
\text { formulae }\left(\Gamma_{1}, \Gamma_{2}\{i / x\}\right) \vDash p\{i / x\}
$$$$
\Gamma_{1}, \Gamma_{2}\{i / x\} \vdash p\{i / x\} \text { true }
$$

Lemma D.8 (context exchange). Let $\Gamma_{1} \vdash D_{1}$ : dtype

$$
\begin{array}{ccc}
\frac{\Gamma_{1}, \Gamma_{2}, x: D_{1} \vdash T: \text { type }}{\Gamma_{1}, x: D_{1}, \Gamma_{2} \vdash T: \text { type }} & \frac{\Gamma_{1}, \Gamma_{2}, x: D_{1} \vdash D: \text { dtype }}{\Gamma_{1}, x: D_{1}, \Gamma_{2} \vdash D: \text { dtype }} & \frac{\Gamma_{1}, \Gamma_{2}, x: D_{1} \vdash p \text { true }}{\Gamma_{1}, x: D_{1}, \Gamma_{2} \vdash p \text { true }} \\
\frac{\Gamma_{1}, \Gamma_{2}, x: D_{1} \vdash p: \text { prop }}{\Gamma_{1}, x: D_{1}, \Gamma_{2} \vdash p: \text { prop }} & \frac{\Gamma_{1}, \Gamma_{2}, x: D_{1} \vdash i: D_{2}}{\Gamma_{1}, x: D_{1}, \Gamma_{2} \vdash i: D_{2}} & \frac{\Gamma_{1}, \Gamma_{2}, x: D: \text { context }}{\Gamma_{1}, x: D, \Gamma_{2}: \text { context }} \\
& \frac{\Gamma_{1}, \Gamma_{2}, x: D_{1} \vdash D_{2}<: D_{3}}{\Gamma_{1}, x: D_{1}, \Gamma_{2} \vdash D_{2}<: D_{3}} &
\end{array}
$$

Proof. By mutual rule induction on the various "main" hypotheses.

\section{D.2 Results related to type equality}

This section is divided in two parts: first, the results regarding definitional type equality are presented in $\S$ D.2.1. Later, $\S$ D.2.2 presents the type checking algorithm for type equality.

\section{D.2.1 Definitional Type Equality}

Main results in this section: agreement for type equality (Lemma D.9) and type equality is an equivalence relation (Lemma D.11).

Lemma D.9 (agreement for type equality).

$$
\frac{\Gamma \vdash T_{1} \equiv T_{2}}{\Gamma \vdash T_{1}: \text { type } \quad \Gamma \vdash T_{2}: \text { type }}
$$

Proof. By rule induction on the derivation of the hypothesis. We must analyse fourteen cases. The congruence/equivalence cases are standard. We detail the other four.

Case $\Gamma \vdash$ skip; $T \equiv T$ :

hyp. premise

1 , agreement for type formation

2 , type formation

$$
\begin{aligned}
& \Gamma \vdash T: \text { type } \\
& \Gamma: \text { context } \\
& \Gamma \vdash \text { skip : type }
\end{aligned}
$$


Case $\Gamma \vdash \forall x \leq i . T \equiv$ skip:

$$
\begin{aligned}
& \text { hyp. premise } \\
& \text { hyp. premise } \\
& \text { 1, type formation } \\
& \text { 2, agreement for true } \\
& \text { 4, type formation }
\end{aligned}
$$$$
\Gamma, x:\{y: \text { int } \mid y \leq i\} \vdash T: \text { type }
$$$$
\Gamma \vdash i<1 \text { true }
$$$$
\Gamma \vdash \forall x \leq i . T: \text { type }
$$$$
\Gamma \text { : context }
$$$$
\Gamma \vdash \text { skip : type }
$$

Case $\Gamma \vdash$ message $i_{1} i_{2} D \equiv$ skip:

hyp. premise
hyp. premise
hyp. premise
2,3, type formation
3, agreement for dtype
5, type formation

$$
\begin{aligned}
& \Gamma \vdash i_{1}, i_{2} \neq \text { rank true } \\
& \Gamma \vdash 1 \leq i_{1}, i_{2} \leq \text { size } \wedge i_{1} \neq i_{2} \text { true } \\
& \Gamma \vdash D: \text { dtype } \\
& \Gamma \vdash \text { message } i_{1} i_{2} D: \text { type } \\
& \Gamma: \text { context } \\
& \Gamma \vdash \text { skip : type }
\end{aligned}
$$

Case $\Gamma \vdash \forall x \leq i . T \equiv(T\{i / x\} ; \forall x \leq i-1 . T)$ :

hyp. premise
hyp. premise
1, inversion
3 , inversion
2 , lemma D.1
5, inversion
6 , inversion
7, lemma D.1

$$
\begin{aligned}
& \Gamma \vdash i \geq 1 \text { true } \\
& \Gamma, x:\{y: \text { int } \mid y \leq i\} \vdash T: \text { type } \\
& \Gamma \vdash i \geq 1: \text { prop } \\
& \Gamma \vdash i: \text { int } \\
& \Gamma, x:\{y: \text { int } \mid y \leq i\}: \text { context } \\
& \Gamma \vdash\{y: \text { int } \mid y \leq i\}: \text { dtype } \\
& \Gamma, y: \text { int } \vdash y \leq i: \text { prop } \\
& \Gamma, y: \text { int }: \text { context }
\end{aligned}
$$

It is always the case that an index term is smaller or equal to itself:

$$
\begin{array}{ll}
\text { 4, deducibility } & \Gamma \vdash i \leq i \text { true } \\
8 \text {, def. of subs. } & \Gamma \vdash(y \leq i)\{i / y\} \text { true }
\end{array}
$$

By application of index formation rules and the substitution lemma (Lemma D.7):

$$
\begin{array}{ll}
4,10, \text { index formation } & \Gamma \vdash i:\{y: \text { int } \mid y \leq i\} \\
11,2, \text { lemma D.7 } & \Gamma \vdash T\{i / x\}: \text { type }
\end{array}
$$

It is easy to prove that $\Gamma \vdash\{y$ : int $\mid y \leq i-1\}<:\{y:$ int $\mid y \leq i\}$ (it follows from 9, standard deducibility, and index formation).

$$
\begin{array}{ll}
2, \text { lemma D.5 } & \Gamma, x:\{y: \text { int } \mid y \leq i-1\} \vdash T: \text { type } \\
16, \text { type formation } & \Gamma \vdash \forall x \leq i-1 . T: \text { type }
\end{array}
$$

Finally, we can join assertions 11 and 13 by applying the type formation rule for sequential composition:

$$
\begin{array}{ll}
11,13, \text { type formation } & \Gamma \vdash T\{i / x\} ; \forall x \leq i-1 . T: \text { type } \\
1,2, \text { type formation } & \Gamma \vdash \forall x \leq i . T: \text { type }
\end{array}
$$


Lemma D.10 (Datatype equality is an equivalence relation).

Proof. Notice that $\Gamma \vdash D \equiv D^{\prime}$ : dtype requires $\Gamma \vdash D<: D^{\prime}$ and $\Gamma \vdash D^{\prime}<: D$. Reflexivity and Transitivity follow from Lemma D.6. Symmetry follows by induction on $\Gamma \vdash D \equiv D^{\prime}$ : dtype.

Lemma D.11 (type equality is an equivalence relation).

Proof. Reflexivity follows from the congruence rules; symmetry and transitivity are built into the definition.

\section{D.2.2 Type Checking Algorithm for Type Equality}

The construction of a type checking algorithm for type equality is divided in three parts: In $\S$ D.2.2 we present the results on structural congruence. § D.2.2 introduces type-term normalization. Finally, § D.2.2 presents the soundness and completeness results of the algorithm.

\section{D.2.2.1 Structural congruence}

We use the structural congruence rules as presented in Figure 19. As usual, we identify terms up-to $\alpha$-equivalence. The main results in this section are agreement for structural congruence (Lemma D.12), that structural congruence is an equivalence relation (Lemma D.13) and that structural congruence is decidable (Lemma D.14).

Lemma D.12 (agreement for $\equiv_{c}$ ).

$$
\frac{\Gamma \vdash T_{1} \equiv_{c} T_{2}}{\Gamma \vdash T_{1}: \text { type } \Gamma \vdash T_{2}: \text { type }}
$$

Proof. Straightforward (the set rules of rules in $\Gamma \vdash T_{1} \equiv_{c} T_{2}$ is a subset of the rules in $\Gamma \vdash T_{1} \equiv T_{2}$ : type, having the same premises. The cases have been already considered in the proof of lemma D.9).

Lemma D.13 ( $\equiv_{c}$ is an equivalence relation).

Proof. Symmetry and transitivity are embedded in the definition of $\equiv_{c}$. Reflexivity follows by rule induction on the typing rules for $\equiv_{c}$, using the first 9 top-bottom rules.

Notice that the monadic rules for structural congruence are the same as the ones for the $\pi$-calculus withouth replication, for which decidability has already been proven [?]. We refer to this result to claim the decidability of structural congruence.

Lemma D.14 ( $\equiv_{c}$ is decidable).

\section{D.2.2.2 Type conversion}

The Type-conversion algorithm is presented in Figure 20. The main results for this section are agreement for type-conversion (lemma 3.1), context subsumption for conversion (lemma D.15), confluence for type conversion (lemma 3.2) and strong normalization (lemma D.16).

Lemma 3.1 (agreement for $\Gamma \vdash T \Rightarrow T^{\prime}$ ). Statement on page 7 .

Proof. By rule induction on the derivation of the hypothesis. The thirteen cases are straightforward. We detail one of them:

Case $\Gamma \vdash \forall x \leq i . T \Rightarrow T^{\prime} ; T^{\prime \prime}$

$\begin{array}{ll}\text { Rule premise } & \Gamma \vdash i \geq 1 \text { true } \\ \text { Rule premise } & \Gamma \vdash T\{i / x\} \Rightarrow T^{\prime} \\ \text { Rule premise } & \Gamma \vdash \forall x \leq i-1 . T \Rightarrow T^{\prime \prime} \\ \text { Rule premise } & \Gamma, x:\{y: \text { int } \mid y \leq i\} \vdash T: \text { type } \\ 2, \mathrm{IH} & \Gamma \vdash T^{\prime}: \text { type } \\ 3, \mathrm{IH} & \Gamma \vdash T^{\prime \prime}: \text { type } \\ 5,6 \text {, formation } & \Gamma \vdash T^{\prime} ; T^{\prime \prime}: \text { type } \\ 4, \text { formation } & \Gamma \vdash \forall x \leq i . T: \text { type }\end{array}$


Lemma D.15 (context subsumption for $\Gamma \vdash T \Rightarrow T^{\prime}$ ).

$$
\frac{\Gamma, x: D_{1} \vdash T \Rightarrow T^{\prime} \quad \Gamma \vdash D_{2}<: D_{1}}{\Gamma, x: D_{2} \vdash T \Rightarrow T^{\prime}}
$$

Proof. By rule induction on the first hypothesis, using Lemma D.5.

Lemma 3.2 ( $\Gamma \vdash T \Rightarrow T^{\prime}$ is confluent). Statement on page 7.

Proof. It follows by rule induction on the first hypothesis.

In order to prove the decidability of algorithmic equality, it is necessary that the type-conversion reduces type terms to a normal form.

Lemma D.16 ( $\Gamma \vdash T \Rightarrow T^{\prime}$ is strongly normalizing).

Proof. The only rule creating new type terms in $\Gamma \vdash T \Rightarrow T^{\prime}$ is the expansion rule for primitive recursion. As primitive recursion is strongly normalizing, we are done.

\section{D.2.2.3 Algorithmic Type Equality}

The type equality algorithm is presented in Figure 18. The main results in this section are agreement for algorithmic type equality (Lemma D.17), soundness (Lemma D.18) and completeness (Lemma D.19) and decidability (Lemma D.20) for algorithmic type equality

Lemma D.17 (agreement for algorithmic type equality).

$$
\frac{\Gamma \vdash T_{1} \equiv_{a} T_{2}}{\Gamma \vdash T_{1}: \text { type } \Gamma \vdash T_{2}: \text { type }}
$$

Proof. From the hypothesis we know that $\Gamma \vdash T_{1} \Rightarrow T_{1}^{\prime}, \Gamma \vdash T_{2} \Rightarrow T_{2}^{\prime}$ and $\Gamma \vdash T_{1}^{\prime} \equiv_{c} T_{2}^{\prime}$. By applying lemma 3.1 in the first two derivations, we know that $\Gamma \vdash T_{1}:$ type and $\Gamma \vdash T_{2}:$ type and we are done.

Theorem D.18 (soundness of the type equality algorithm).

$$
\frac{\Gamma \vdash T_{1} \equiv_{a} T_{2}}{\Gamma \vdash T_{1} \equiv T_{2}}
$$

Proof. By inversion of the hypothesis $\Gamma \vdash T_{1} \equiv_{a} T_{2}$ we know that:

$$
\begin{aligned}
& \Gamma \vdash T_{1} \Rightarrow T_{1}^{\prime} \\
& \Gamma \vdash T_{2} \Rightarrow T_{2}^{\prime} \\
& \Gamma \vdash T_{1}^{\prime} \equiv_{c} T_{2}^{\prime}
\end{aligned}
$$

We proceed by rule induction on (2a) followed by rule induction on (2b). We show some of the cases.

Case $\Gamma \vdash$ message $i_{1} i_{2} D_{1} \Rightarrow$ message $i_{1} i_{2} D_{1}$ :

$$
\begin{array}{ll}
\text { rule } & \Gamma \vdash \text { message } i_{1} i_{2} D_{1} \Rightarrow \text { message } i_{1} i_{2} D_{1} \\
1, \text { inversion } & \Gamma \nvdash i_{1}, i_{2} \neq \text { rank true } \\
1, \text { inversion } & \Gamma \vdash 1 \leq i_{1}, i_{2} \leq \text { size } \wedge i_{1} \neq i_{2} \text { true } \\
1, \text { inversion } & \Gamma \vdash D_{1}: \text { dtype }
\end{array}
$$

Applying rule induction on (2b), we have only one case to consider (all other rule inductions are void in 2c):

$$
\begin{array}{ll}
\text { rule } & \Gamma \vdash \text { message } i_{3} i_{4} D_{2} \Rightarrow \text { message } i_{3} i_{4} D_{2} \\
5, \text { inversion } & \Gamma \nvdash i_{3}, i_{4} \neq \text { rank true } \\
5 \text {, inversion } & \Gamma \vdash 1 \leq i_{3}, i_{4} \leq \text { size } \wedge i_{3} \neq i_{4} \text { true } \\
5 \text {, inversion } & \Gamma \vdash D_{2}: \text { dtype }
\end{array}
$$




$$
\begin{array}{ll}
1,5 \text {, eq. (2c) } & \Gamma \vdash \text { message } i_{1} i_{2} D_{1} \equiv_{c} \text { message } i_{3} i_{4} D_{2} \\
9, \text { inversion } & \Gamma \vdash i_{1}, i_{2}=i_{3}, i_{4} \text { true } \\
9, \text { inversion } & \Gamma \vdash D_{1} \equiv D_{2}: \text { dtype } \\
2,3,10,11 & \Gamma \vdash \text { message } i_{1} i_{2} D_{1} \equiv \text { message } i_{3} i_{4} D_{2}
\end{array}
$$

Case skip, reduce, scatter, gather: Ibid.

Case $\Gamma \vdash p ? T_{1}: T_{2} \Rightarrow p ? T_{1}^{\prime}: T_{2}^{\prime}$ :

$$
\begin{array}{ll}
\text { rule } & \Gamma \vdash p_{!} ? T_{1}: T_{2} \Rightarrow p_{1} ? T_{1}^{\prime}: T_{2}^{\prime} \\
1, \text { inversion } & \Gamma \vdash p_{1}: \text { prop } \\
1, \text { inversion } & \Gamma \vdash T_{1} \Rightarrow T_{1}^{\prime} \\
1, \text { inversion } & \Gamma \vdash T_{2} \Rightarrow T_{2}^{\prime}
\end{array}
$$

Applying rule induction on (2b), we have only one case to consider (all other rule inductions are void in 2c):

$$
\begin{array}{ll}
\text { rule } & \Gamma \vdash p_{2} ? T_{3}: T_{4} \Rightarrow p_{2} ? T_{3}^{\prime}: T_{4}^{\prime} \\
\text { 5, inversion } & \Gamma \vdash p_{2}: \text { prop } \\
\text { 5, inversion } & \Gamma \vdash T_{3} \Rightarrow T_{3}^{\prime} \\
\text { 5, inversion } & \Gamma \vdash T_{4} \Rightarrow T_{4}^{\prime} \\
\text { 1, 5, eq. (2c) } & \Gamma \vdash p_{1} ? T_{1}^{\prime}: T_{2}^{\prime} \equiv_{c} p_{2} ? T_{3}^{\prime}: T_{4}^{\prime} \\
\text { 9, inversion } & \Gamma \vdash p_{1} \leftrightarrow p_{2} \text { true } \\
\text { 9, inversion } & \Gamma \vdash T_{1}^{\prime} \equiv_{c} T_{3}^{\prime} \\
\text { 9, inversion } & \Gamma \vdash T_{2}^{\prime} \equiv_{c} T_{4}^{\prime} \\
\text { 3, 7, 11, IH } & \Gamma \vdash T_{1} \equiv T_{3} \\
4,8,12, \text { IH } & \Gamma \vdash T_{2} \equiv T_{4} \\
10,13,14, \equiv \text { formation } & \Gamma \vdash p_{1} ? T_{1}: T_{2} \equiv p_{2} ? T_{3}: T_{4}
\end{array}
$$

Case val, broadcast, congruence for $\forall x \leq i . T$ : Ibid.

Case projection ( $\Gamma \vdash$ message $i_{1} i_{2} D_{1} \Rightarrow$ skip):

$$
\begin{array}{ll}
\text { rule } & \Gamma \vdash \text { message } i_{1} i_{2} D_{1} \Rightarrow \text { skip } \\
1, \text { inversion } & \Gamma \vdash i_{1}, i_{2} \neq \text { rank true } \\
1, \text { inversion } & \Gamma \vdash 1 \leq i_{1}, i_{2} \leq \text { size } \wedge i_{1} \neq i_{2} \text { true } \\
\text { 1, inversion } & \Gamma \vdash D_{1}: \text { dtype } \\
\text { 4, lemma D.1 } & \Gamma: \text { context }
\end{array}
$$

We then proceed by applying rule induction on $(2 b)$. We have to consider the following cases:

Subcase $\Gamma \vdash$ skip $\Rightarrow$ skip:

$$
\begin{array}{ll}
\text { rule } & \Gamma \vdash \text { skip } \Rightarrow \text { skip } \\
1,6, \text { eq. (2c) } & \Gamma \vdash \text { skip } \equiv_{c} \text { skip } \\
1,6,7, \text { IH } & \Gamma \vdash \text { message } i_{1} i_{2} D_{1} \equiv \text { skip }
\end{array}
$$

Subcase $\Gamma \vdash$ message $i_{3} i_{4} D_{2} \Rightarrow$ skip:

$$
\begin{array}{ll}
\text { rule } & \Gamma \vdash \text { message } i_{3} i_{4} D_{2} \Rightarrow \text { skip } \\
\text { 6, inversion } & \Gamma \vdash i_{3}, i_{4} \neq \text { rank true } \\
\text { 6, inversion } & \Gamma \vdash 1 \leq i_{3}, i_{4} \leq \text { size } \wedge i_{3} \neq i_{4} \text { true } \\
\text { 6, inversion } & \Gamma \vdash D_{2}: \text { dtype } \\
\text { 1, 6, eq. (2c) } & \Gamma \vdash \text { skip } \equiv_{c} \text { skip }
\end{array}
$$


Subcase $\Gamma \vdash \forall x \leq i . T \Rightarrow$ skip:

$$
\begin{array}{ll}
\text { rule } & \Gamma \vdash \forall x \leq i . T \Rightarrow \text { skip } \\
\text { 6, inversion } & \Gamma \vdash i<1 \text { true } \\
\text { 6, inversion } & \Gamma, x:\{y: \text { int } \mid y \leq i\} \vdash T: \text { type } \\
\text { 1, 6, eq. (2c) } & \Gamma \vdash \text { skip } \equiv_{c} \text { skip } \\
1,6,9, \mathrm{IH} & \Gamma \vdash \text { message } i_{1} i_{2} D_{1} \equiv_{c} \forall x \leq i . T
\end{array}
$$

Case primitive recursion, base case $(\Gamma \vdash \forall x \leq i . T \Rightarrow$ skip): Ibid

Case primitive recursion, induction case $\left(\Gamma \vdash \forall x \leq i . T_{1} \Rightarrow T_{2} ; T_{3}\right)$ :

$$
\begin{array}{ll}
\text { rule } & \Gamma \vdash \forall x_{1} \leq i_{1} \cdot T_{1} \Rightarrow T_{2} ; T_{3} \\
1, \text { inversion } & \Gamma \vdash i_{1} \geq 1 \text { true } \\
1, \text { inversion } & \Gamma \vdash T_{1}\left\{i_{1} / x_{1}\right\} \Rightarrow T_{2} \\
1, \text { inversion } & \Gamma \vdash \forall x_{1} \leq i_{1}-1 . T_{1} \Rightarrow T_{3} \\
1, \text { inversion } & \Gamma \vdash \forall x_{1} \leq i_{1} . T_{1}: \text { type }
\end{array}
$$

We then proceed by applying rule induction on (2b). We have to consider the following cases:

Subcase sequential composition $\left(\Gamma \vdash T_{4} ; T_{5} \Rightarrow T_{4}^{\prime} ; T_{5}^{\prime}\right)$ :

$$
\begin{array}{ll}
\text { rule } & \Gamma \vdash T_{4} ; T_{5} \Rightarrow T_{4}^{\prime} ; T_{5}^{\prime} \\
\text { 6, inversion } & \Gamma \vdash T_{4} \Rightarrow T_{4}^{\prime} \\
\text { 6, inversion } & \Gamma \vdash T_{5} \Rightarrow T_{5}^{\prime} \\
\text { 1, 6, eq. (2c) } & \Gamma \vdash T_{2} ; T_{3} \equiv_{c} T_{4}^{\prime} ; T_{5}^{\prime} \\
\text { 9, inversion } & \Gamma \vdash T_{2} \equiv_{c} T_{4}^{\prime} \\
\text { 9, inversion } & \Gamma \vdash T_{3} \equiv_{c} T_{5}^{\prime} \\
\text { 3, 7, 10, IH } & \Gamma \vdash T_{1}\left\{i_{1} / x_{1}\right\} \equiv T_{4} \\
\text { 4, 8, 11, IH } & \Gamma \vdash \forall x_{1} \leq i_{1}-1 . T_{1} \equiv T_{5} \\
\text { 12, 13, } \equiv \text { formation } & \Gamma \vdash T_{1}\left\{i_{1} / x_{1}\right\} ; \forall x_{1} \leq i_{1}-1 . T_{1} \equiv T_{4} ; T_{5} \\
\text { 1, lemma 3.1 } & \Gamma \vdash \forall x_{1} \leq i_{1} \cdot T_{1}: \text { type } \\
\text { 15, inversion } & \Gamma, x_{1}:\left\{y_{1}: \text { int } \mid y_{1} \leq i_{1}\right\} \vdash T_{1}: \text { type } \\
\text { 2, 16, } \equiv \text { formation } & \Gamma \vdash \forall x_{1} \leq i_{1} . T_{1} \equiv T_{1}\left\{i_{1} / x_{1}\right\} ; \forall x_{1} \leq i_{1}-1 . T_{1} \\
\text { 14, 17, lemma D.11 } & \Gamma \vdash \forall x_{1} \leq i_{1} . T_{1} \equiv T_{4} ; T_{5}
\end{array}
$$

Subcase primitive recursion, induction case $\left(\Gamma \vdash \forall x_{2} \leq i_{2} . T_{4} \Rightarrow T_{5} ; T_{6}\right)$ :

$$
\begin{array}{ll}
\text { rule } & \Gamma \vdash \forall x_{2} \leq i_{2} \cdot T_{4} \Rightarrow T_{5} ; T_{6} \\
6, \text { inversion } & \Gamma \vdash i_{2} \geq 1 \text { true } \\
\text { 6, inversion } & \Gamma \vdash T_{4}\left\{i_{2} / x_{2}\right\} \Rightarrow T_{5} \\
\text { 6, inversion } & \Gamma \vdash \forall x_{2} \leq i_{2}-1 . T_{4} \Rightarrow T_{6} \\
\text { 6, inversion } & x_{2} \notin \mathrm{fv}\left(T_{5}\right) \\
\text { 1, 6, eq. (2c) } & \Gamma \vdash T_{2} ; T_{3} \equiv_{c} T_{5} ; T_{6} \\
\text { 11, inversion } & \Gamma \vdash T_{2} \equiv_{c} T_{5} \\
\text { 11, inversion } & \Gamma \vdash T_{3} \equiv_{c} T_{6} \\
3,8,12, \text { IH } & \Gamma \vdash T_{1}\left\{i_{1} / x_{1}\right\} \equiv T_{4}\left\{i_{2} / x_{2}\right\} \\
4,9,13, \text { IH } & \Gamma \vdash \forall x_{1} \leq i_{1}-1 . T_{1} \equiv \forall x_{2} \leq i_{2}-1 . T_{4}
\end{array}
$$


1, lemma 3.1

16 , inversion

6, lemma 3.1

18 , inversion

$2,17, \equiv$ formation

$8,19, \equiv$ formation

14,15 , 三 formation

20, 21, 22, lemma D.11
$\Gamma \vdash \forall x_{1} \leq i_{1} . T_{1}:$ type

$$
\Gamma, x_{1}:\left\{y_{1}: \text { int } \mid y_{1} \leq i_{1}\right\} \vdash T_{1}: \text { type }
$$$$
\Gamma \vdash \forall x_{2} \leq i_{2} \cdot T_{4}: \text { type }
$$$$
\Gamma, x_{2}:\left\{y_{2}: \text { int } \mid y_{2} \leq i_{2}\right\} \vdash T_{4} \text { : type }
$$$$
\Gamma \vdash \forall x_{1} \leq i_{1} . T_{1} \equiv T_{1}\left\{i_{1} / x_{1}\right\} ; \forall x_{1} \leq i_{1}-1 . T_{1}
$$$$
\Gamma \vdash \forall x_{2} \leq i_{2} . T_{4} \equiv T_{4}\left\{i_{2} / x_{2}\right\} ; \forall x_{2} \leq i_{2}-1 . T_{4}
$$$$
\Gamma \vdash T_{1}\left\{i_{1} / x_{1}\right\} ; \forall x_{1} \leq i_{1}-1 . T_{1} \equiv T_{4}\left\{i_{2} / x_{2}\right\} ; \forall x_{2} \leq i_{2}-1 . T_{4}
$$$$
\Gamma \vdash \forall x_{1} \leq i_{1} \cdot T_{1} \equiv \forall x_{2} \leq i_{2} \cdot T_{4}
$$

Case $\left(\Gamma \vdash T_{1} ; T_{2} \Rightarrow T_{1}^{\prime} ; T_{2}^{\prime}\right):$ Ibid.

Theorem D.19 (completeness of the type equality algorithm).

$$
\frac{\Gamma \vdash T_{1} \equiv T_{2}}{\Gamma \vdash T_{1} \equiv_{a} T_{2}}
$$

Proof. We proceed by rule induction on the hypothesis. Notice that the premises to conclude $\Gamma \vdash T_{1} \equiv_{a} T_{2}$ are $\Gamma \vdash T_{1} \Rightarrow T_{1}^{\prime}$, $\Gamma \vdash T_{2} \Rightarrow T_{2}^{\prime}$ and $\Gamma \vdash T_{1}^{\prime} \equiv_{c} T_{2}^{\prime}$. We proceed to prove that we can derive such premises in each case.

Case $\Gamma \vdash$ broadcast $i_{1} x: D_{1} \cdot T_{1} \equiv$ broadcast $i_{2} x: D_{2} \cdot T_{2}:$ type.

$$
\begin{array}{ll}
\text { rule premise } & \Gamma \vdash i_{1}=i_{2} \text { true } \\
\text { rule premise } & \Gamma \vdash D_{1} \equiv D_{2} \\
\text { rule premise } & \Gamma, x: D_{1} \vdash T_{1} \equiv T_{2} \\
\text { rule premise } & \Gamma \vdash 1 \leq i_{1} \leq \text { size true } \\
3, \mathrm{IH} & \Gamma, x: D_{1} \vdash T_{1} \Rightarrow T_{3} \\
3, \mathrm{IH} & \Gamma, x: D_{1} \vdash T_{2} \Rightarrow T_{4} \\
3, \mathrm{IH} & \Gamma, x: D_{1} \vdash T_{3} \equiv{ }_{c} T_{4} \\
4,5, \text { formation } & \Gamma \vdash \text { broadcast } i_{1} x: D_{1} \cdot T_{1} \Rightarrow \text { broadcast } i_{1} x: D_{1} \cdot T_{3} \\
2,3, \text { lemma D.15 } & \Gamma, x: D_{2} \vdash T_{2} \Rightarrow T_{4} \\
1,4,9, \text { formation } & \Gamma \vdash \text { broadcast } i_{2} x: D_{2} \cdot T_{2} \Rightarrow \text { broadcast } i_{2} x: D_{2} \cdot T_{4} \\
1,2,4,7, \text { formation } & \Gamma \vdash \text { broadcast } i_{1} x: D_{1} \cdot T_{3} \equiv{ }_{c} \text { broadcast } i_{2} x: D_{2} \cdot T_{4}
\end{array}
$$

Case Congruence rules for skip, message, scatter, gather, reduce, val, $p$ ? $T: T^{\prime}, T ; T^{\prime}$ and primitive recursion: Ibid. Case projection rule, $\Gamma \vdash$ message $i_{1} i_{2} D \equiv$ skip.

$$
\begin{array}{ll}
\text { rule premise } & \Gamma \vdash i_{1}, i_{2} \neq \text { rank true } \\
\text { rule premise } & \Gamma \vdash 1 \leq i_{1}, i_{2} \leq \text { size } \wedge i_{1} \neq i_{2} \text { true } \\
\text { rule premise } & \Gamma \vdash D: \text { dtype } \\
2,3 \text {, type formation } & \Gamma \vdash \text { message } i_{1} i_{2} D: \text { type } \\
1,4, \Rightarrow \text { formation } & \Gamma \vdash \text { message } i_{1} i_{2} D \Rightarrow \text { skip } \\
4, \text { lemma D. } 1 & \Gamma: \text { context } \\
6, \Rightarrow \text { formation } & \Gamma \vdash \text { skip } \Rightarrow \text { skip } \\
6, \equiv \text { formation } & \Gamma \vdash \text { skip }{ }_{c} \text { skip }
\end{array}
$$

Case primitive recursion, base case $(\Gamma \vdash \forall x \leq i . T \equiv$ skip $)$.

rule premise

rule premise

2 , type formation

$$
\begin{aligned}
& \Gamma \vdash i<1 \text { true } \\
& \Gamma, x:\{y: \text { int } \mid y \leq i\} \vdash T: \text { type } \\
& \Gamma \vdash \forall x \leq i . T: \text { type }
\end{aligned}
$$




$$
\begin{array}{ll}
1,3, \Rightarrow \text { formation } & \Gamma \vdash \forall x \leq i . T \Rightarrow \text { skip } \\
4, \text { lemma } 3.1 & \Gamma \vdash \text { skip }: \text { type } \\
5, \text { inversion } & \Gamma: \text { context } \\
6, \Rightarrow \text { formation } & \Gamma \vdash \text { skip } \Rightarrow \text { skip } \\
6, \equiv \text { formation } & \Gamma \vdash \text { skip } \equiv_{c} \text { skip }
\end{array}
$$

Case primitive recursion, induction case $(\Gamma \vdash \forall x \leq i . T \equiv(T\{i / x\} ; \forall x \leq i-1 . T))$.

$\begin{array}{ll}\text { rule } & \Gamma \vdash \forall x \leq i . T_{1} \equiv\left(T_{1}\{i / x\} ; \forall x \leq i-1 . T_{1}\right) \\ \text { 1, inversion } & \Gamma \vdash i \geq 1 \text { true } \\ \text { 1, inversion } & \Gamma, x:\{y: \text { int } \mid y \leq i\} \vdash T_{1}: \text { type } \\ \text { 1, lemma D.9 } & \Gamma \vdash \forall x \leq i . T_{1}: \text { type } \\ \text { 1, lemma D.9 } & \Gamma \vdash T_{1}\{i / x\} ; \forall x \leq i-1 . T_{1}: \text { type } \\ \text { 5, inversion } & \Gamma \vdash T_{1}\{i / x\}: \text { type } \\ \text { 5, inversion } & \Gamma \vdash \forall x \leq i-1 . T_{1}: \text { type } \\ \text { 6, lemma D.11 } & \Gamma \vdash T_{1}\{i / x\} \equiv T_{1}\{i / x\} \\ \text { 8, IH } & \Gamma \vdash T_{1}\{i / x\} \Rightarrow T_{2} \\ \text { 8, IH } & \Gamma \vdash T_{1}\{i / x\} \Rightarrow T_{3} \\ \text { 8, IH } & \Gamma \vdash T_{2} \equiv_{c} T_{3} \\ \text { 9, 10, lemma 3.2 } & T_{2}=T_{3} \\ \text { 7, lemma D.11 } & \Gamma \vdash \forall x \leq i-1 . T_{1} \equiv \forall x \leq i-1 . T_{1} \\ \text { 13, IH } & \Gamma \vdash \forall x \leq i-1 . T_{1} \Rightarrow T_{4} \\ \text { 13, IH } & \Gamma \vdash \forall x \leq i-1 . T_{1} \Rightarrow T_{5} \\ \text { 13, IH } & \Gamma \vdash T_{4} \equiv_{c} T_{5} \\ \text { 14, 15, lemma 3.2 } & T_{4}=T_{5} \\ \text { 9, 14, } \Rightarrow \text { formation } & \Gamma \vdash T_{1}\{i / x\} ; \forall x \leq i-1 . T_{1} \Rightarrow T_{2} ; T_{4} \\ \text { 2, 4, 9, 14, } \Rightarrow \text { formation } & \Gamma \vdash \forall x \leq i . T_{1} \Rightarrow T_{2} ; T_{4} \\ \text { 20, lemma 3.1 } & \Gamma \vdash T_{2} ; T_{4}: \text { type } \\ \text { 21, lemma D.13 } & \Gamma \vdash T_{2} ; T_{4} \equiv_{c} T_{2} ; T_{4} \\ & \end{array}$

Case monoidal rules, $\Gamma \vdash T$; skip $\equiv T$ (the left rule is similar).

$$
\begin{array}{ll}
\text { rule premise } & \Gamma \vdash T: \text { type } \\
\text { 1, lemma D.11 } & \Gamma \vdash T \equiv T \\
\text { 2, IH } & \Gamma \vdash T \Rightarrow T^{\prime} \\
\text { 2, IH } & \Gamma \vdash T \Rightarrow T^{\prime \prime} \\
\text { 2, IH } & \Gamma \vdash T^{\prime} \equiv_{c} T^{\prime \prime} \\
\text { 3,4, lemma 3.2 } & T^{\prime}=T^{\prime \prime} \\
\text { 2, lemma D.9 } & \Gamma \vdash T: \text { type } \\
\text { 7, lemma D.1 } & \Gamma: \text { context } \\
\text { 8, } \Rightarrow \text { formation } & \Gamma \vdash \text { skip } \Rightarrow \text { skip } \\
\text { 3,9, f formation } & \Gamma \vdash T \text {; skip } \Rightarrow T^{\prime} ; \text { skip } \\
\text { 3, lemma 3.1 } & \Gamma \vdash T^{\prime}: \text { type } \\
\text { 11, 三 formation } & \Gamma \vdash T^{\prime} \text {; skip } \equiv_{c} T^{\prime}
\end{array}
$$

Case monoidal rules, associativity $\Gamma \vdash T_{1} ;\left(T_{2} ; T_{3}\right) \equiv\left(T_{1} ; T_{2}\right) ; T_{3}$.

$$
\text { rule premise }
$$

$\Gamma \vdash T_{1}, T_{2}, T_{3}:$ type 


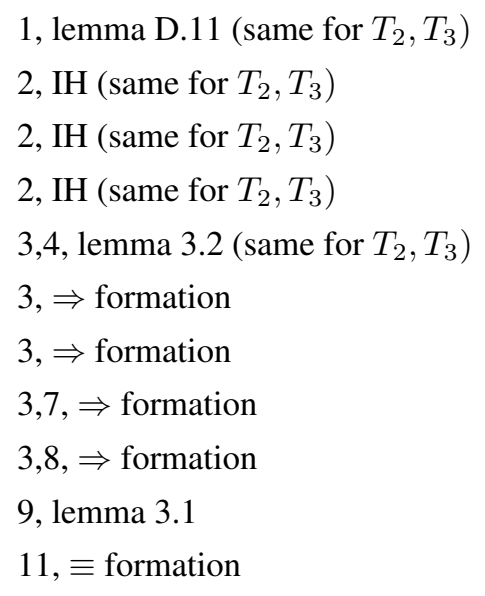

$$
\begin{aligned}
& \Gamma \vdash T_{1} \equiv T_{1} \\
& \Gamma \vdash T_{1} \Rightarrow T_{1}^{\prime} \\
& \Gamma \vdash T_{1} \Rightarrow T_{1}^{\prime \prime} \\
& \Gamma \vdash T_{1}^{\prime} \equiv_{c} T_{1}^{\prime \prime} \\
& T_{1}^{\prime}=T_{1}^{\prime \prime} \\
& \Gamma \vdash T_{1} ; T_{2} \Rightarrow T_{1}^{\prime} ; T_{2}^{\prime} \\
& \Gamma \vdash T_{2} ; T_{3} \Rightarrow T_{2}^{\prime} ; T_{3}^{\prime} \\
& \Gamma \vdash\left(T_{1} ; T_{2}\right) ; T_{3} \Rightarrow\left(T_{1}^{\prime} ; T_{2}^{\prime}\right) ; T_{3}^{\prime} \\
& \Gamma \vdash T_{1} ;\left(T_{2} ; T_{3}\right) \Rightarrow T_{1}^{\prime} ;\left(T_{2}^{\prime} ; T_{3}^{\prime}\right) \\
& \Gamma \vdash T_{1}^{\prime}, T_{2}^{\prime}, T_{3}^{\prime}: \text { type } \\
& \Gamma \vdash\left(T_{1}^{\prime} ; T_{2}^{\prime}\right) ; T_{3}^{\prime} \equiv_{c} T_{1}^{\prime} ;\left(T_{2}^{\prime} ; T_{3}^{\prime}\right)
\end{aligned}
$$

Case symmetry, $\Gamma \vdash T_{1} \equiv T_{2}$.

$$
\begin{aligned}
& \text { rule premise } \\
& 1, \mathrm{IH} \\
& 1, \mathrm{IH} \\
& 1, \mathrm{IH} \\
& \text { 4, lemma D.13 }
\end{aligned}
$$

$$
\begin{aligned}
& \Gamma \vdash T_{2} \equiv T_{1} \\
& \Gamma \vdash T_{2} \Rightarrow T_{2}^{\prime} \\
& \Gamma \vdash T_{1} \Rightarrow T_{1}^{\prime} \\
& \Gamma \vdash T_{2}^{\prime} \equiv_{c} T_{1}^{\prime} \\
& \Gamma \vdash T_{1}^{\prime} \equiv_{c} T_{2}^{\prime}
\end{aligned}
$$

Case transitivity.

rule premise
rule premise
$1, \mathrm{IH}$
$1, \mathrm{IH}$
$1, \mathrm{IH}$
$2, \mathrm{IH}$
$2, \mathrm{IH}$
$2, \mathrm{IH}$
4,6, lemma 3.2
9,8
$10,5, \equiv$ formation

$$
\begin{aligned}
& \Gamma \vdash T_{1} \equiv T_{2} \\
& \Gamma \vdash T_{2} \equiv T_{3} \\
& \Gamma \vdash T_{1} \Rightarrow T_{1}^{\prime} \\
& \Gamma \vdash T_{2} \Rightarrow T_{2}^{\prime} \\
& \Gamma \vdash T_{1}^{\prime} \equiv_{c} T_{2}^{\prime} \\
& \Gamma \vdash T_{2} \Rightarrow T_{2}^{\prime \prime} \\
& \Gamma \vdash T_{3} \Rightarrow T_{3}^{\prime} \\
& \Gamma \vdash T_{2}^{\prime \prime} \equiv_{c} T_{3}^{\prime} \\
& T_{2}^{\prime}=T_{2}^{\prime \prime} \\
& \Gamma \vdash T_{2}^{\prime} \equiv_{c} T_{3}^{\prime} \\
& \Gamma \vdash T_{1}^{\prime} \equiv_{c} T_{3}^{\prime}
\end{aligned}
$$

Theorem D.20 (Decidability of algorithmic type equality).

Proof. Follows as a consequence of lemmas D.14 and D.16.

\section{D.3 Results related to program types}

Main result in this section: agreement for program type formation (Lemma D.21).

Lemma D.21 (agreement for program type formation).

$$
\frac{T_{1}, \ldots, T_{n}: \text { ptype }}{\Gamma^{n, 1} \vdash T_{1}: \text { type } \quad \ldots \quad \Gamma^{n, n} \vdash T_{n}: \text { type }}
$$

Proof. By inversion on the hypothesis, we know that $\Gamma^{n, k} \vdash T_{k} \equiv T$ : type, $(1 \leq k \leq n)$. The proof follows by the application of lemma D.9. 


\section{D.4 Results related to references}

Main results in this section: agreement for type formation, now with references (Lemma D.22), index term subtyping remains a pre-order (Lemma D.23) and that deducibility contains no references (Lemma D.24).

Lemma D.22 (agreement for type formation, with references). The statement of this lemma is that of Lemma D.1, page 26.

Proof. By mutual rule induction on the hypotheses, reusing the cases from the proof of Lemma D.1.

Lemma D.23 (subtyping is still a pre-order). The statement of this lemma is that of Lemma D.6, page 27.

Proof. By rule induction on the hypothesis, reusing the cases from the proof of Lemma D.6. Inversion for <: (Lemma D.4) must be extended with the following case.

5. If $\Gamma \vdash D_{1}$ ref $<: D_{2}$ then $D_{2}$ is $D_{3}$ ref and $\Gamma \vdash D_{1}<: D_{3}$ or $D_{2}$ is $\left\{x: D_{3} \mid p\right\}$ and $\Gamma \vdash D_{1}$ ref $<: D_{3}$ and $\Gamma, x: D_{1}$ ref $\vdash p$ true.

Lemma D.24 (deducibility contains no references).

$$
\frac{\Gamma \vdash p \text { true }}{r \notin \operatorname{refs}(p)}
$$

Proof. Assertion $\Gamma \vdash p$ true is not defined on reference identifiers.

\section{D.5 Results related to expressions}

Main results in this section: agreement for expression formation (Lemma D.25), weakening for expression formation (Lemma D.26), strengthening for expression formation (Lemma D.27), context subsumption for expression formation (Lemma D.28), substitution in expression formation (Lemma D.29), context exchange for expression formation (Lemma D.30), and the inversion lemma for expression formation (Lemma D.31).

Lemma D.25 (agreement for expression formation).

$$
\frac{\Gamma \vdash e: T}{\Gamma \vdash T: \text { type }}
$$

Proof. By rule induction on the hypothesis, using Lemma D.1.

Lemma D.26 (weakening for expression formation).

$$
\frac{\Gamma \vdash e: T \quad \Gamma \vdash D: \text { dtype }}{\Gamma, x: D \vdash e: T}
$$

Proof. By rule induction on the first hypothesis, using Lemma D.2.

Lemma D.27 (strengthening for expression formation).

$$
\frac{\Gamma, x: D \vdash e: T \quad x \notin \mathrm{fv}(e, T)}{\Gamma \vdash e: T}
$$

Proof. By rule induction on the first hypothesis, using Lemma D.3.

Lemma D.28 (context subsumption for expression formation).

$$
\frac{\Gamma, x: D_{1} \vdash e: T \quad \Gamma \vdash D_{2}<: D_{1}}{\Gamma, x: D_{2} \vdash e: T}
$$

Proof. By rule induction on the first hypothesis, using Lemma D.5.

Lemma D.29 (substitution for expression formation).

$$
\frac{\Gamma, x: D \vdash e: T \quad \Gamma \vdash i: D}{\Gamma \vdash e\{i / x\}: T\{i / x\}}
$$

Proof. By rule induction on the first hypothesis, using Lemma D.7. 
Lemma D.30 (context exchange for expression formation).

$$
\frac{\Gamma_{1}, \Gamma_{2}, x: D_{1} \vdash e: T \quad \Gamma_{1} \vdash D_{1}: \text { dtype }}{\Gamma_{1}, x: D_{1}, \Gamma_{2} \vdash e: T}
$$

Proof. By rule induction on the first hypothesis, using Lemma D.8.

The lemma below establishes the various expressions that may inhabit a given type. It forms the basis to the proof of the result on progress (Theorem 4.6).

Lemma D.31 (inversion for expression formation).

1. If $\Gamma \vdash e$ : broadcast $i x: D . T$ then $e$ is either

- let $x: D_{1}=$ broadcast $i_{1} i_{2}$ in $e_{1}$ and $\Gamma \vdash D_{1} \equiv D$ : dtype and $\Gamma \vdash i_{1}=i$ true and $\Gamma \vdash 1 \leq i_{1} \leq$ size true and $\Gamma \vdash i_{2}: D$ and rank $\notin \mathrm{fv}\left(i_{1}\right)$ and $\Gamma, x: D \vdash e_{1}: T$, or

- let $y: D_{1}=i_{1}$ in $e_{1}$ and $\Gamma \vdash i_{1}: D_{1}$ and $y \notin \mathrm{fv}($ broadcast $i x: D . T)$ and $\Gamma, y: D_{1} \vdash e_{1}:$ broadcast $i x: D . T$, or

- if $p$ then $e_{1}$ else $e_{2}$ and $\Gamma, p \vdash e_{1}$ : broadcast $i x: D . T$ and $\Gamma, \neg p \vdash e_{2}$ : broadcast $i x: D . T$, or

- $e_{1} ; e_{2}$ and $\Gamma \vdash e_{1}$ : skip and $\Gamma \vdash e_{2}:$ broadcast $i x: D . T$.

2. If $\Gamma \vdash e$ : message $i i^{\prime} D$ then $e$ is either

- send $i_{1} i_{2}$ and $\Gamma \vdash$ rank $=i$ true and $\Gamma \vdash i_{1}=i^{\prime}$ true and $\Gamma \vdash i_{2}: D$, or

- receive $i_{1} i_{2}$ and $\Gamma \vdash i_{1}=i$ true and $\Gamma \vdash$ rank $=i^{\prime}$ true, and $\Gamma \vdash i_{2}: D$ ref, or

- let $y: D_{1}=i_{1}$ in $e_{1}$ and $\Gamma \vdash i_{1}: D_{1}$ and $y \notin \mathrm{fv}\left(\right.$ message $\left.i i^{\prime} D\right)$ and $\Gamma, y: D_{1} \vdash e_{1}$ : message $i i^{\prime} D$, or

- if $p$ then $e_{1}$ else $e_{2}$ and $\Gamma, p \vdash e_{1}$ : message $i i^{\prime} D$ and $\Gamma, \neg p \vdash e_{2}$ : message $i i^{\prime} D$, or

- $e_{1} ; e_{2}$ and $\Gamma \vdash e_{1}$ : skip and $\Gamma \vdash e_{2}$ : message $i i^{\prime} D$.

3. If $\Gamma \vdash e: \forall x \leq i . T$ then $e$ is either

- for $x: i_{1} . .1$ do $e_{1}$ and $\Gamma \vdash i_{1} \leq i$ true and $\Gamma, x:\{y:$ int $\mid y \leq i\} \vdash e_{1}: T$ and $\Gamma \vdash T\{i / x\}=T\{i-1 / x\}=\cdots=\left\{i_{1}+1 / x\right\} \equiv$ skip : type, or

- skip and $\Gamma \vdash i<1$ true, or

- let $y: D_{1}=i_{1}$ in $e_{1}$ and $\Gamma \vdash i_{1}: D_{1}$ and $y \notin \mathrm{fv}(\forall x \leq i . T)$ and $\Gamma, y: D_{1} \vdash e_{1}: \forall x \leq i . T$, or

- if $p$ then $e_{1}$ else $e_{2}$ and $\Gamma, p \vdash e_{1}: \forall x \leq i . T$ and $\Gamma, \neg p \vdash e_{2}: \forall x \leq i . T$, or

- $e_{1} ; e_{2}$ and $\Gamma \vdash e_{1}:$ skip and $\Gamma \vdash e_{2}: \forall x \leq i . T$, or

4. If $\Gamma \vdash e:\left(T ; T^{\prime}\right)$ then $e$ is either

- $e_{1} ; e_{2}$ and $\Gamma \vdash e_{1}: T$ and $\Gamma \vdash e_{2}: T^{\prime}$

- for $x: i_{1} . .1$ do $e_{1}$ and $\Gamma \vdash i_{1} \geq 1$ true and $\Gamma, x:\left\{y:\right.$ int $\left.\mid y \leq i_{1}\right\} \vdash e_{1}: T_{1}$ and $\Gamma \vdash T_{1}\{i / x\} \equiv T:$ type and $\Gamma \vdash\left(\forall x \leq i_{1}-1 . T_{1}\right) \equiv T^{\prime}$ : type, or

- let $y: D_{1}=i_{1}$ in $e_{1}$ and $\Gamma \vdash i_{1}: D_{1}$ and $y \notin \mathrm{fv}\left(T ; T^{\prime}\right)$ and $\Gamma, y: D_{1} \vdash e_{1}: T ; T^{\prime}$, or

- if $p$ then $e_{1}$ else $e_{2}$ and $\Gamma, p \vdash e_{1}: T ; T^{\prime}$ and $\Gamma, \neg p \vdash e_{2}: T ; T^{\prime}$, or

- $e_{1} ; e_{2}$ and $\Gamma \vdash e_{1}:$ skip and $\Gamma \vdash e_{2}: T ; T^{\prime}$, or

5. If $\Gamma \vdash e$ : skip then $e$ is either

- skip, or

- while $p$ do $e_{1}$ and $\Gamma \vdash p$ : prop and $\Gamma, p \vdash e_{1}$ : skip, or

- for $x: i . .1$ do $e_{1}$ and $\Gamma \vdash i<1$ true and $\Gamma, x:\{y:$ int $\mid y \leq i\} \vdash e_{1}:$ skip, or

- let $y: D_{1}=i_{1}$ in $e_{1}$ and $\Gamma \vdash i_{1}: D_{1}$ and $y \notin \mathrm{fv}\left(\right.$ skip) and $\Gamma, y: D_{1} \vdash e_{1}$ : skip, or

- if $p$ then $e_{1}$ else $e_{2}$ and $\Gamma, p \vdash e_{1}$ : skip and $\Gamma, \neg p \vdash e_{2}$ : skip, or

- $e_{1} ; e_{2}$ and $\Gamma \vdash e_{1}$ : skip and $\Gamma \vdash e_{2}$ : skip.

6. If $\Gamma \vdash e$ : gather $i D$ then $e$ is either

- gather $i_{1} i_{2} i_{3}$ and $\Gamma \vdash i_{1}=i$ true and $\Gamma \vdash 1 \leq i_{1} \leq$ size true and $\Gamma \vdash i_{2}:\left\{x: D\right.$ array $\left.\mid \operatorname{len}\left(i_{3}\right)=\operatorname{size} * \operatorname{len}(x)\right\}$ and $\Gamma \vdash i_{3}: D$ array ref, or

- let $y: D_{1}=i_{1}$ in $e_{1}$ and $\Gamma \vdash i_{1}: D_{1}$ and $y \notin \mathrm{fv}($ gather $i D)$ and $\Gamma, y: D_{1} \vdash e_{1}:$ gather $i D$, or

- if $p$ then $e_{1}$ else $e_{2}$ and $\Gamma, p \vdash e_{1}:$ gather $i D$ and $\Gamma, \neg p \vdash e_{2}$ : gather $i D$, or

- $e_{1} ; e_{2}$ and $\Gamma \vdash e_{1}$ : skip and $\Gamma \vdash e_{2}$ : gather $i D$.

7. If $\Gamma \vdash e$ : scatter $i D$ then $e$ is either

- scatter $i_{1} i_{2} i_{3}$ and $\Gamma \vdash i_{1}=i$ true and $\Gamma \vdash 1 \leq i_{1} \leq$ size true and $\Gamma \vdash i_{2}:\left\{x: D\right.$ array $\left.\mid \operatorname{len}\left(i_{3}\right)=\operatorname{size} * \operatorname{len}(x)\right\}$ and $\Gamma \vdash i_{3}: D$ array ref, or 
- let $y: D_{1}=i_{1}$ in $e_{1}$ and $\Gamma \vdash i_{1}: D_{1}$ and $y \notin \mathrm{fv}(\operatorname{scatter} i D)$ and $\Gamma, y: D_{1} \vdash e_{1}:$ scatter $i D$, or

- if $p$ then $e_{1}$ else $e_{2}$ and $\Gamma, p \vdash e_{1}$ : scatter $i D$ and $\Gamma, \neg p \vdash e_{2}$ : scatter $i D$, or

- $e_{1} ; e_{2}$ and $\Gamma \vdash e_{1}$ : skip and $\Gamma \vdash e_{2}$ : scatter $i D$.

8. If $\Gamma \vdash e$ : reduce $i$ then $e$ is either

- reduce $i_{1} i_{2} i_{3}$ and $\Gamma \vdash i_{1}=i$ true and $\Gamma \vdash 1 \leq i_{1} \leq$ size true and $\Gamma \vdash i_{2}:$ float and $\Gamma \vdash i_{3}:$ float ref, or

- let $y: D_{1}=i_{1}$ in $e_{1}$ and $\Gamma \vdash i_{1}: D_{1}$ and $y \notin \mathrm{fv}\left(\right.$ reduce $\left.i_{1}\right)$ and $\Gamma, y: D_{1} \vdash e_{1}$ : reduce $i_{1}$, or

- if $p$ then $e_{1}$ else $e_{2}$ and $\Gamma, p \vdash e_{1}$ : reduce $i_{1}$ and $\Gamma, \neg p \vdash e_{2}$ : reduce $i_{1}$, or

- $e_{1} ; e_{2}$ and $\Gamma \vdash e_{1}$ : skip and $\Gamma \vdash e_{2}$ : reduce $i_{1}$.

9. If $\Gamma \vdash e:$ val $x: D . T$ then $e$ is either

- let $x: D_{1}=$ val $i_{1}$ in $e_{1}$ and $\Gamma \vdash D_{1} \equiv D:$ dtype and $\Gamma \vdash i_{1}: D$ and $\Gamma, x: D \vdash e_{1}: T$

- let $y: D_{1}=i_{1}$ in $e_{1}$ and $\Gamma \vdash i_{1}: D_{1}$ and $y \notin \mathrm{fv}(\operatorname{val} x: D . T)$ and $\Gamma, y: D_{1} \vdash e_{1}:$ val $x: D . T$, or

- if $p$ then $e_{1}$ else $e_{2}$ and $\Gamma, p \vdash e_{1}:$ val $x: D . T$ and $\Gamma, \neg p \vdash e_{2}:$ val $x: D . T$, or

- $e_{1} ; e_{2}$ and $\Gamma \vdash e_{1}$ : skip and $\Gamma \vdash e_{2}:$ val $x: D . T$.

10. If $\Gamma \vdash e: p$ ? $T: T^{\prime}$ then $e$ is either

- ifc $p^{\prime}$ then $e_{1}$ else $e_{2}$ and $\Gamma \vdash p \leftrightarrow p^{\prime}$ true and $\Gamma \vdash e_{1}: T$ and $\Gamma \vdash e_{2}: T^{\prime}$ and rank $\notin \mathrm{fv}(p)$, or

- let $y: D_{1}=i_{1}$ in $e_{1}$ and $\Gamma \vdash i_{1}: D_{1}$ and $y \notin \mathrm{fv}(p$ ? T:T' $)$ and $\Gamma, y: D_{1} \vdash e_{1}: p$ ? $T: T^{\prime}$, or

- if $p$ then $e_{1}$ else $e_{2}$ and $\Gamma, p \vdash e_{1}: p$ ? $T: T^{\prime}$ and $\Gamma, \neg p \vdash e_{2}: p$ ? $T: T^{\prime}$, or

- $e_{1} ; e_{2}$ and $\Gamma \vdash e_{1}:$ skip and $\Gamma \vdash e_{2}: p ? T: T^{\prime}$.

Proof. By a case analysis on the rules for expression formation.

\section{D.6 Results related to stores}

Main results in this section: agreement for store-to-context formation (Lemma D.32), agreement for index evaluation (Lemma D.37) and that evaluation induces deducibility (Lemma D.38).

Lemma D.32 (agreement for store-to-context conversion).

$$
\frac{\rho \text { to } \Gamma}{\rho: \text { store } \Gamma: \text { context }}
$$

Proof. By rule induction on the hypothesis.

Lemma D.33 (store-to-context inversion).

$$
\frac{\rho: \text { store }}{\rho \text { to } \Gamma}
$$

Proof. By rule induction on the hypothesis.

Lemma D.34 (store update does not affect store-to-context).

$$
\frac{\rho \text { to } \Gamma \quad \Gamma^{n}, \Gamma \vdash r: D \text { ref } \Gamma^{n}, \Gamma \vdash v: D}{\rho[r:=v] \text { to } \Gamma}
$$

Proof. By rule induction on the hypothesis $\rho$ to $\Gamma$.

Lemma D.35 (store value is index).

$$
\frac{\rho \vdash r: D \text { ref } \quad r:=v \in \rho}{\rho \vdash v: D}
$$

Proof. From the second hypothesis we know that $\rho$ is not empty. Case $\rho$ is $\rho^{\prime}, r:=v$. From the definition of $\rho$ to $\Gamma$ we know that $\left(\rho^{\prime}, r:=v\right)$ to $\left(\Gamma^{\prime}, r: D\right.$ ref). By inversion we get $\Gamma^{\prime} \vdash v: D$. Applying weakening (Lemma D.2) we conclude $\Gamma^{\prime}, r: D$ ref $\vdash v: D$. Case $\rho$ is $\rho^{\prime}, r^{\prime}:=v^{\prime}$ with $r \neq r^{\prime}$ follows by induction.

Lemma D.36 (inversion of store formation).

$$
\frac{\rho \text { to } \Gamma \quad r: D \in \Gamma}{r:=v \in \rho \quad D \text { is } D^{\prime} \text { ref } \quad \Gamma \vdash v: D^{\prime}}
$$


Proof. By rule induction on hypothesis $\rho$ to $\Gamma$. From the second premise we know that $\Gamma$ is not empty, then the only case to consider is when $\rho$ is not empty.

Case $\rho$ is $\rho^{\prime}, r:=v^{\prime}$ :

$$
\begin{aligned}
& \text { store as context } \\
& 1 \text {, inversion } \\
& 1 \text {, inversion } \\
& 1 \text {, inversion } \\
& 1 \\
& 3 \text {, lemma D. } 2
\end{aligned}
$$

$$
\begin{aligned}
& \rho^{\prime}, r:=v^{\prime} \text { to } \Gamma^{\prime}, r: D^{\prime} \text { ref } \\
& \rho^{\prime} \text { to } \Gamma^{\prime} \\
& \Gamma^{\prime} \vdash v^{\prime}: D^{\prime} \\
& r \notin \rho^{\prime}, \Gamma, D^{\prime} \\
& r:=v^{\prime} \in \rho^{\prime}, r:=v^{\prime} \\
& \Gamma^{\prime}, r: D^{\prime} \text { ref } \vdash v^{\prime}: D^{\prime}
\end{aligned}
$$

Case $\rho$ is $\rho^{\prime}, r^{\prime}:=v^{\prime}$ and $r \neq r^{\prime}$ :

Case hyp., store as context

$$
\begin{aligned}
& \rho^{\prime}, r^{\prime}:=v^{\prime} \text { to } \Gamma^{\prime}, r^{\prime}: D^{\prime} \text { ref } \\
& r \neq r^{\prime} \\
& r: D \in \Gamma^{\prime}, r^{\prime}: D^{\prime} \text { ref } \\
& r: D \in \Gamma^{\prime} \\
& r: D \in \Gamma^{\prime} \\
& r:=v^{\prime \prime} \in \rho^{\prime} \\
& D \text { is } D^{\prime \prime} \text { ref } \\
& \Gamma^{\prime} \vdash v: D^{\prime \prime}
\end{aligned}
$$$$
4,5 \text {, induction }
$$

Lemma D.37 (agreement for evaluation).

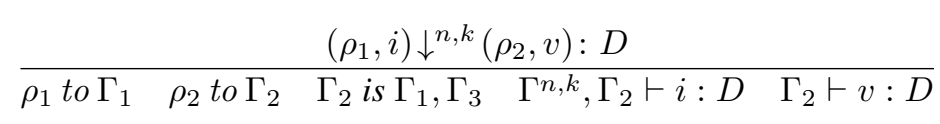

Proof. By rule induction on the hypothesis. We list the most representative cases.

Case $(\rho, f) \downarrow^{n, k}(\rho, f)$ : float

$$
\begin{aligned}
& \text { rule premise } \\
& \text { 1, lemma D.33 } \\
& \text { 2, lemma D.32 } \\
& \text { 3, index formation } \\
& \text { 4, lemmas D.2, D.8 }
\end{aligned}
$$

$$
\begin{aligned}
& \rho: \text { store } \\
& \rho \text { to } \Gamma \\
& \Gamma: \text { context } \\
& \Gamma \vdash f: \text { float } \\
& \Gamma^{n, k}, \Gamma \vdash f: \text { float }
\end{aligned}
$$

Case $(\rho$, size $) \downarrow^{n, k}(\rho, n)$ : int

$$
\begin{aligned}
& \text { rule premise } \\
& \text { 1, lemma D.33 } \\
& \text { 2, lemma D.32 } \\
& \text { 3, lemmas D.2,D.8 } \\
& \text { 4, index formation } \\
& \text { 2, index formation }
\end{aligned}
$$

$$
\begin{aligned}
& \rho: \text { store } \\
& \rho \text { to } \Gamma \\
& \Gamma: \text { context } \\
& \Gamma^{n, k}, \Gamma: \text { context } \\
& \Gamma^{n, k}, \Gamma \vdash \text { size }: \text { int } \\
& \Gamma \vdash n: \text { int }
\end{aligned}
$$

Case $(\rho, r) \downarrow^{n, k}(\rho, r): D$ ref

$$
\begin{aligned}
& \rho: \text { store } \\
& r:=v \in \rho
\end{aligned}
$$


From (2) we know that $\rho$ is non empty. Subcase $\rho$ is $\rho^{\prime}, r:=v$ :

$\begin{array}{ll}\text { 1, lemma D.33 } & \left(\rho^{\prime}, r:=v\right) \text { to }\left(\Gamma^{\prime}, r: D \text { ref }\right) \\ \text { 4, lemma D.32 } & \Gamma^{\prime}, r: D \text { ref }: \text { context } \\ \text { 5, index formation } & \Gamma^{\prime}, r: D \text { ref } \vdash r: D \text { ref } \\ \text { 6, lemmas D.2,D.8 } & \Gamma^{n, k}, \Gamma^{\prime}, r: D \text { ref } \vdash r: D \text { ref }\end{array}$

The subcase where $\rho$ is $\rho^{\prime}, r^{\prime}:=v^{\prime}$ with $r \neq r^{\prime}$ follows by induction.

Case $\left(\rho_{1}, ! i\right) \downarrow^{n, k}\left(\rho_{2}, v\right): D$

rule premise
rule premise
1 , induction
1 , induction
1 , induction
1 , induction
1 , induction
6, index formation
$2,3,7$, lemma D.35

$$
\begin{aligned}
& \left(\rho_{1}, i\right) \downarrow^{n, k}\left(\rho_{2}, r\right): D \text { ref } \\
& r:=v \in \rho_{2} \\
& \rho_{2} \text { to } \Gamma_{2} \\
& \rho_{1} \text { to } \Gamma_{1} \\
& \Gamma_{2} \text { is } \Gamma_{1}, \Gamma_{3} \\
& \Gamma^{n, k}, \Gamma_{2} \vdash i: D \text { ref } \\
& \Gamma_{2} \vdash r: D \text { ref } \\
& \Gamma^{n, k}, \Gamma_{2} \vdash ! i: D \\
& \Gamma_{2} \vdash v: D
\end{aligned}
$$

Case $\left(\rho_{1}, i_{1}:=i_{2}\right) \downarrow^{n, k}\left(\rho_{2}[r:=v], v\right): D$

$$
\begin{aligned}
& \text { rule premise } \\
& \text { rule premise } \\
& 1 \text {, induction } \\
& 1 \text {, induction } \\
& 1 \text {, induction } \\
& 1 \text {, induction } \\
& 1 \text {, induction } \\
& 2 \text {, induction } \\
& 2 \text {, induction } \\
& 2 \text {, induction } \\
& 2 \text {, induction }
\end{aligned}
$$

$$
\begin{aligned}
& \left(\rho_{1}, i_{1}\right) \downarrow^{n, k}\left(\rho_{3}, r\right): D \text { ref } \\
& \left(\rho_{3}, i_{2}\right) \downarrow^{n, k}\left(\rho_{2}, v\right): D \\
& \rho_{1} \text { to } \Gamma_{1} \\
& \Gamma^{n, k}, \Gamma_{3} \vdash i_{1}: D \text { ref } \\
& \Gamma_{3} \vdash r: D \text { ref } \\
& \rho_{3} \text { to } \Gamma_{3} \\
& \Gamma_{3} \text { is } \Gamma_{1}, \Gamma_{4} \\
& \Gamma^{n, k}, \Gamma_{2} \vdash i_{2}: D \\
& \Gamma_{2} \vdash v: D \\
& \Gamma_{2} \text { is } \Gamma_{3}, \Gamma_{5} \\
& \rho_{2} \text { to } \Gamma_{2}
\end{aligned}
$$

Through simple context manipulation and the fact that store update does not affect store to context formation (Lemma D.34) we obtain the remaining conclusions:

$$
\begin{aligned}
& 7,10 \text {, equals for equals } \\
& 4,12 \text {, lemma D.2 } \\
& 8,13 \text {, index formation } \\
& 5,10, \text { lemma D. } 2 \\
& 8, \text { lem. D.1, inv. } \\
& 9,16, \text { lem. D.2, D. } \\
& 15,16, \text { lem. D. } 2, \text { D. } 8 \\
& 11,17,18, \text { lemma D.34 }
\end{aligned}
$$

$$
\begin{aligned}
& \Gamma_{2} \text { is } \Gamma_{1}, \Gamma_{4}, \Gamma_{5} \\
& \Gamma^{n, k}, \Gamma_{2} \vdash i_{1}: D \text { ref } \\
& \Gamma^{n, k}, \Gamma_{2} \vdash i_{1}:=i_{2}: D \\
& \Gamma_{2} \vdash r: D \text { ref } \\
& \Gamma^{n, k}: \text { context } \\
& \Gamma^{n, k}, \Gamma_{2} \vdash v: D \\
& \Gamma^{n, k}, \Gamma_{2} \vdash r: D \text { ref } \\
& \rho_{2}[r:=v] \text { to } \Gamma_{2}
\end{aligned}
$$


Case $\left(\rho_{1}\right.$, mkref $\left.i\right) \downarrow^{n, k}\left(\left(\rho_{2}, r:=v\right), r\right): D$ ref

$\begin{array}{ll}\text { rule premise } & \left(\rho_{1}, i\right) \downarrow^{n, k}\left(\rho_{2}, v\right): D \\ \text { rule premise } & r \text { fresh } \\ 1, \text { induction } & \Gamma^{n, k}, \Gamma_{2} \vdash i: D \\ 1, \text { induction } & \Gamma_{2} \vdash v: D \\ 1, \text { induction } & \Gamma_{2} \text { is } \Gamma_{1}, \Gamma_{3} \\ 1, \text { induction } & \rho_{1} t o \Gamma_{1} \\ 1, \text { induction } & \rho_{2} \text { to } \Gamma_{2} \\ 3, \text { index formation } & \Gamma^{n, k}, \Gamma_{2} \vdash \text { mkref } i: D \\ 7, \text { lemma D.32 } & \rho_{2}: \text { store } \\ 7, \text { lemma D.32 } & \Gamma_{2}: \text { context } \\ 2,10,7,4, \text { store formation } & \rho_{2}, r:=v: \text { store } \\ 11, \text { store as context formation } & \rho_{2}, r:=v \text { to } \Gamma_{2}, r: D \text { ref } \\ 5, \text { monotonicity } & \Gamma_{2}, r:=v \text { is } \Gamma_{1}, \Gamma_{3}, r:=v\end{array}$

Case $\left(\rho_{1},\left[i_{1}, \ldots, i_{n}\right]\right) \downarrow^{n, k}\left(\rho_{n+1},\left[v_{1}, \ldots, v_{n}\right]\right): D$ array

$$
\begin{array}{ll}
\text { rule premise } & \left(\rho_{1}, i_{1}\right) \downarrow^{n, k}\left(\rho_{2}, v_{1}\right): D \\
\text { rule premise } & \left(\rho_{n}, i_{n}\right) \downarrow^{n, k}\left(\rho_{n+1}, v_{n}\right): D \\
2, \text { induction, for all } 1 \leq l \leq n & \Gamma^{n, k}, \Gamma_{l+1} \vdash i_{l}: D \\
2, \text { induction, for all } 1 \leq l \leq n & \Gamma_{l+1} \vdash v_{l}: D \\
2, \text { induction, for all } 2 \leq l \leq n & \Gamma_{l+1} \text { is } \Gamma_{l}, \Gamma_{l}^{\prime} \\
2, \text { induction, for all } 1 \leq l \leq n+1 & \rho_{l} \text { to } \Gamma_{l}
\end{array}
$$

By applying basic lemmas over contexts (weakening, strengthening), index formation rules, and transitivity we get:

$$
\begin{aligned}
& 5, \text { transitivity } \\
& 3,7, \text { lemma D. } 2 \\
& \text { 8, index formation } \\
& \text { 4, } 7 \text {, lemma D. } 2 \\
& \text { 11, index formation }
\end{aligned}
$$

$$
\begin{aligned}
& \Gamma_{l+1}=\Gamma_{1}, \Gamma_{1}^{\prime}, \ldots, \Gamma_{n}^{\prime} \\
& \Gamma^{n, k}, \Gamma_{l+1} \vdash i_{l}: D \\
& \Gamma^{n, k}, \Gamma_{l+1} \vdash\left[i_{1}, \ldots, i_{n}\right]: D \text { array } \\
& \Gamma_{l+1} \vdash v_{l}: D \\
& \Gamma_{l+1} \vdash\left[v_{1}, \ldots, v_{n}\right]: D \text { array }
\end{aligned}
$$

Case $\left(\rho_{1}, i_{1}\left[i_{2}\right]\right) \downarrow^{n, k}\left(\rho_{3}, v_{m}\right): D$

$$
\begin{aligned}
& \text { rule premise } \\
& \text { rule premise } \\
& 1 \text {, induction } \\
& 1 \text {, induction } \\
& 1 \text {, induction } \\
& 1 \text {, induction } \\
& 1 \text {, induction } \\
& 2 \text {, induction } \\
& 2 \text {, induction } \\
& 2 \text {, induction } \\
& 2 \text {, induction } \\
& 10,15 \text {, equals for equals }
\end{aligned}
$$

$$
\begin{aligned}
& \left(\rho_{1}, i_{1}\right) \downarrow^{n, k}\left(\rho_{2},\left[v_{1}, \ldots, v_{l}\right]\right):\{x: D \text { array } \mid \text { len }(x)=l\} \\
& \left(\rho_{2}, i_{2}\right) \downarrow^{n, k}\left(\rho_{3}, m\right):\{x: \text { int } \mid 1 \leq x \leq l\} \\
& \Gamma^{n, k}, \Gamma_{2} \vdash i_{1}:\{D \text { array } \mid \text { len }(x)=l\} \\
& \Gamma_{2} \vdash\left[v_{1}, \ldots, v_{l}\right]:\{D \text { array } \mid \operatorname{len}(x)=l\} \\
& \rho_{1} \text { to } \Gamma_{1} \\
& \Gamma_{2}=\Gamma_{1}, \Gamma_{4} \\
& \rho_{2} \text { to } \Gamma_{2} \\
& \Gamma^{n, k}, \Gamma_{3} \vdash i_{2}:\{x: \text { int } \mid 1 \leq x \leq l\} \\
& \Gamma_{3} \vdash m:\{x: \text { int } \mid 1 \leq x \leq l\} \\
& \rho_{3} \text { to } \Gamma_{3} \\
& \Gamma_{3}=\Gamma_{2}, \Gamma_{5} \\
& \Gamma_{3} \text { is } \Gamma_{1}, \Gamma_{4}, \Gamma_{5}
\end{aligned}
$$




$$
\begin{array}{ll}
\text { 8, inversion, lemma D.2 } & \Gamma^{n, k}, \Gamma_{3} \vdash 1 \leq i_{1} \leq l \text { true } \\
\text { 3, 13, index formation } & \Gamma^{n, k}, \Gamma_{3} \vdash i_{1}\left[i_{2}\right]: D \\
\text { 8, inversion, lemma D.2 } & \Gamma_{3} \vdash v_{m}: D
\end{array}
$$

Case refinement introduction.

$$
\begin{aligned}
& \text { rule premise } \\
& \text { rule premise } \\
& 1 \text {, induction } \\
& 1 \text {, induction } \\
& 1 \text {, induction } \\
& 1 \text {, induction } \\
& 1 \text {, induction } \\
& 2, \text { lemma D. } 2 \\
& 6,8, \text { index formation } \\
& 2, \text { lemma D. } 2 \\
& 7,10 \text {, index formation }
\end{aligned}
$$

$$
\begin{aligned}
& \left(\rho_{1}, i\right) \downarrow^{n, k}\left(\rho_{2}, v\right): D \\
& \varepsilon \vdash p\{i / x\} \text { true } \\
& \rho_{1} \text { to } \Gamma_{1} \\
& \rho_{2} \text { to } \Gamma_{2} \\
& \Gamma_{2} \text { is } \Gamma_{1}, \Gamma_{3} \\
& \Gamma^{n, k}, \Gamma_{2} \vdash i: D \\
& \Gamma_{2} \vdash v: D \\
& \Gamma^{n, k}, \Gamma_{2} \vdash p\{i / x\} \text { true } \\
& \Gamma^{n, k}, \Gamma_{2} \vdash i:\{x: D \mid p\} \\
& \Gamma_{2} \vdash p\{i / x\} \text { true } \\
& \Gamma_{2} \vdash v:\{x: D \mid p\}
\end{aligned}
$$

Case subtyping.

rule premise
rule premise
1 , induction
1 , induction
1 , induction
1 , induction
1 , induction
2,6, lemma D.2, subtyping
2,7, subtyping

$$
\begin{aligned}
& \left(\rho_{1}, i\right) \downarrow^{n, k}\left(\rho_{2}, v\right): D_{1} \\
& \rho_{2} \vdash D_{2}<: D_{1} \\
& \rho_{1} \text { to } \Gamma_{1} \\
& \rho_{2} \text { to } \Gamma_{2} \\
& \Gamma_{1} \text { is } \Gamma_{1}, \Gamma_{3} \\
& \Gamma^{n, k}, \Gamma_{2} \vdash i: D_{1} \\
& \Gamma_{2} \vdash v: D_{1} \\
& \Gamma^{n, k}, \Gamma_{2} \vdash i: D_{2} \\
& \Gamma_{2} \vdash v: D_{2}
\end{aligned}
$$

Lemma D.38 (eval to deducibility).

$$
\frac{i \downarrow^{n} m}{\Gamma^{n} \vdash i=m \text { true }}
$$

Proof. By rule induction on the hypothesis, making use of basic assumptions on deducibility such as, $\Gamma \vdash i_{1}=m_{1}$ true and $\Gamma \vdash i_{2}=m_{2}$ true and $m$ is $m_{1}+m_{2}$ implies $\Gamma \vdash i_{1}+i_{2}=m$ true.

Lemma D.39 (evaluation succeeds).

$$
\frac{\Gamma^{n, k}, \rho_{1} \vdash i: D}{\left(\rho_{1}, i\right) \downarrow^{n, k}\left(\rho_{2}, v\right): D}
$$

Proof. By rule induction on the hypothesis. We highlight a few representative cases.

$\begin{array}{ll}\text { premise } & \rho \text { to } \Gamma \\ 1, \text { lemma D.32 } & \rho: \text { store } \Gamma: \text { context }\end{array}$

Case $\Gamma^{n, k}, \Gamma \vdash x: D$

premise

$x: D \in\left(\Gamma^{n, k}, \Gamma\right)$ 
Subcase $x$ is size

$$
1 \text {, eval rule }
$$

$$
(\rho, \text { size }) \downarrow^{n, k}(\rho, n) \text { : int }
$$

Subcase $x$ is rank. As above.

Case $\Gamma^{n, k}, \Gamma \vdash r: D$

$$
\begin{aligned}
& \text { premise } \\
& 1,3 \text {, lemma D.36 } \\
& 2,4 \text {, eval rule }
\end{aligned}
$$

$$
\begin{aligned}
& r: D \in\left(\Gamma^{n, k}, \Gamma\right) \\
& D \text { is } D^{\prime} \text { ref } \wedge \Gamma^{n, k}, \Gamma \vdash v: D^{\prime} \wedge r:=v \in \rho \\
& (\rho, r) \downarrow^{n, k}(\rho, r): D
\end{aligned}
$$

Case $\Gamma^{n, k}, \Gamma \vdash i_{1}+i_{2}:$ int

$$
\begin{aligned}
& \text { premise } \\
& \text { premise } \\
& 3, \text { induction } \\
& 5, \text { lemma D.37 } \\
& 5, \text { lemma D.37 } \\
& 4,6 \text {, lemma D.2 } \\
& 9, \text { induction } \\
& 5,9, \text { eval rule }
\end{aligned}
$$

$$
\begin{aligned}
& \Gamma^{n, k}, \Gamma \vdash i_{1}: \text { int } \\
& \Gamma^{n, k}, \Gamma \vdash i_{2}: \text { int } \\
& \left(\rho, i_{1}\right) \downarrow^{n, k}\left(\rho_{1}, m_{1}\right): \text { int } \\
& \rho_{1} \text { to } \Gamma_{1} \\
& \Gamma_{1} \text { is } \Gamma, \Gamma^{\prime} \\
& \Gamma^{n, k}, \Gamma_{1} \vdash i_{2}: \text { int } \\
& \left(\rho_{1}, i_{2}\right) \downarrow^{n, k}\left(\rho_{2}, m_{2}\right): \text { int } \\
& \left(\rho, i_{1}+i_{2}\right) \downarrow^{n, k}\left(\rho_{2}, v_{1}+v_{2}\right): \text { int }
\end{aligned}
$$

Case $\Gamma^{n, k}, \Gamma \vdash i_{1}\left[i_{2}\right]: D$

premise
premise
4, inversion
4, 5 , index formation
3 , induction
7, lemma D.37
7, lemma D.37
6,9, lemma D.2
10, induction
7,11 , evaluation

Case Subtyping $\Gamma^{n, k}, \Gamma \vdash i: D_{1}$

$$
\begin{aligned}
& \text { premise } \\
& \text { premise } \\
& 3 \text {, induction } \\
& 5 \text {, lemma D.37 } \\
& 5 \text {, lemma D.37 } \\
& 4,7, \text { lemma D.2 } \\
& 5,8, \text { evaluation }
\end{aligned}
$$

$$
\begin{aligned}
& \Gamma^{n, k}, \Gamma \vdash i_{1}:\{x: D \text { array } \mid \text { len }(x)=l\} \\
& \Gamma^{n, k}, \Gamma \vdash 1 \leq i_{2} \leq l \text { true } \\
& \Gamma^{n, k}, \Gamma \vdash i_{2}: \text { int } \\
& \Gamma^{n, k}, \Gamma \vdash i_{2}:\{y: \text { int } \mid 1 \leq y \leq l\} \\
& \left(\rho_{0}, i_{1}\right) \downarrow^{n, k}\left(\rho_{1}, v_{1}\right):\{x: D \text { array } \mid \operatorname{len}(x)=l\} \\
& \rho_{1} \text { to } \Gamma_{1} \\
& \Gamma_{1} \text { is } \Gamma, \Gamma^{\prime} \\
& \Gamma^{n, k}, \Gamma_{1} \vdash i_{2}:\{y: \text { int } \mid 1 \leq y \leq l\} \\
& \left(\rho_{1}, i_{2}\right) \downarrow^{n, k}\left(\rho_{2}, m\right):\{y: \text { int } \mid 1 \leq y \leq l\} \\
& \left(\rho, i_{1}\left[i_{2}\right]\right) \downarrow^{n, k}\left(\rho_{2}, v_{m}\right): D
\end{aligned}
$$

$$
\begin{aligned}
& \Gamma^{n, k}, \Gamma \vdash i: D_{2} \\
& \Gamma^{n, k}, \Gamma \vdash D_{1}<: D_{2} \\
& (\rho, i) \downarrow^{n, k}\left(\rho^{\prime}, v\right): D_{2} \\
& \rho^{\prime} \text { to } \Gamma_{2} \\
& \Gamma_{2} \text { is } \Gamma, \Gamma^{\prime} \\
& \Gamma^{n, k}, \Gamma_{2} \vdash D_{1}<: D_{2} \\
& (\rho, i) \downarrow^{n, k}\left(\rho^{\prime}, v\right): D_{1}
\end{aligned}
$$

Case Refinement $\Gamma^{n, k}, \Gamma \vdash i:\{x: D \mid p\}$

$$
\text { premise }
$$

$$
\Gamma^{n, k}, \Gamma \vdash i: D
$$




$$
\begin{aligned}
& \text { premise } \\
& 3 \text {, induction } \\
& 5 \text {, lemma D.37 } \\
& 5 \text {, lemma D.37 } \\
& 4,7, \text { lemma D.2 } \\
& 5,8 \text {, evaluation }
\end{aligned}
$$

Case $\Gamma \vdash \operatorname{mkref} i: D$ ref

$$
\begin{aligned}
& \text { premise } \\
& 3 \text {, induction } \\
& \text { ref. ids are countable } \\
& 4,6 \text {, eval. }
\end{aligned}
$$

$$
\begin{aligned}
& \Gamma^{n, k}, \Gamma \vdash p\{i / x\} \text { true } \\
& (\rho, i) \downarrow^{n, k}\left(\rho^{\prime}, v\right): D \\
& \rho^{\prime} \text { to } \Gamma_{2} \\
& \Gamma_{2} \text { is } \Gamma, \Gamma^{\prime} \\
& \Gamma^{n, k}, \Gamma_{2} \vdash p\{i / x\} \text { true } \\
& (\rho, i) \downarrow^{n, k}\left(\rho^{\prime}, v\right):\{x: D \mid p\}
\end{aligned}
$$

$$
\begin{aligned}
& \Gamma^{n, k}, \Gamma \vdash i: D \\
& (\rho, i) \downarrow^{n, k}\left(\rho^{\prime}, v\right): D \\
& r \notin \rho^{\prime} \\
& (\rho, \text { mkref } i) \downarrow^{n, k}\left(\left(\rho^{\prime}, r:=v\right), r\right): D^{\prime} \text { ref }
\end{aligned}
$$

Case $\Gamma \vdash ! i: D$

$$
\begin{aligned}
& \Gamma^{n, k}, \Gamma \vdash i: D \text { ref } \\
& (\rho, i) \downarrow^{n, k}\left(\rho^{\prime}, r\right): D \text { ref } \\
& \rho^{\prime} t o \Gamma^{\prime} \\
& \Gamma^{\prime} \vdash r: D \text { ref } \\
& r: D \text { ref } \in \Gamma^{\prime} \\
& r:=v \in \rho^{\prime} \\
& (\rho, ! i) \downarrow^{n, k}\left(\rho^{\prime}, v\right): D
\end{aligned}
$$

Case $\Gamma \vdash i_{1}:=i_{2}: D$

$$
\begin{aligned}
& \text { premise } \\
& \text { premise } \\
& 3 \text {, induction } \\
& \text { 4, lemma D.37 } \\
& \text { 4, lemma D.37 } \\
& 4,7, \text { lemma D.2 } \\
& \text { 8, induction } \\
& 5,9, \text { evaluation }
\end{aligned}
$$

$$
\begin{aligned}
& \Gamma^{n, k}, \Gamma \vdash i_{1}: D \text { ref } \\
& \Gamma^{n, k}, \Gamma \vdash i_{2}: D \\
& \left(\rho, i_{1}\right) \downarrow^{n, k}\left(\rho^{\prime}, r\right): D \text { ref } \\
& \rho^{\prime} \text { to } \Gamma^{\prime} \\
& \Gamma^{\prime} \text { is } \Gamma, \Gamma^{\prime \prime} \\
& \Gamma^{n, k}, \Gamma^{\prime} \vdash i_{2}: D \\
& \left(\rho^{\prime}, i_{2}\right) \downarrow^{n, k}\left(\rho^{\prime \prime}, r\right): D \\
& \left(\rho, i_{1}:=i_{2}\right) \downarrow^{n, k}\left(\rho^{\prime \prime}[r:=v], v\right): D
\end{aligned}
$$

\section{D.7 Results related to processes}

Main results in this section: agreement for process formation (Lemma D.40), agreement for process reduction (Lemma 4.1) and progress for processes (Lemma 4.3).

Lemma D.40 (agreement for process formation).

$$
\frac{\Gamma \vdash q: T}{\Gamma \vdash T: \text { type }}
$$

Proof. From the hypothesis and the only process formation rule, we know that $q$ is $(\rho, e)$ and that $\Gamma, \rho \vdash e: T$. Agreement (Lemma D.1) tells us that $\Gamma, \rho \vdash T$ : type. The premise of all expression formation rules tells us that $T$ contains no ref types. From strengthening (Lemma D.3) we get the result.

Lemma 4.1 (agreement for process reduction). Statement on page 9. 
Proof. By rule induction on the hypothesis. We have nine simple cases, and illustrate one of them.

$$
\begin{array}{ll} 
& (\rho, \text { for } x: i . .1 \text { do } e) \rightarrow^{n, k}(\rho,(e\{i / x\} ; \text { for } x: i-1 . .1 \text { do } e)) \\
\text { rule premise } & \Gamma^{n, k}, \rho \vdash i \geq 1 \text { true } \\
\text { rule premise } & \Gamma^{n, k}, \rho, x:\{y: \text { int } \mid y \leq i\} \vdash e: T
\end{array}
$$

The type for the left hand side follows from the application of expression and process formation rules:

$$
\begin{array}{ll}
3, \text { exp. formation } & \Gamma^{n, k}, \rho \vdash \text { for } x: i . .1 \text { do } e: \forall x \leq i . T \\
4, \text { proc. formation } & \Gamma^{n, k} \vdash(\rho, \text { for } x: i . .1 \text { do } e): \forall x \leq i . T
\end{array}
$$

The right hand side requires slightly more effort.

$$
\begin{array}{ll}
\text { 2, inversion } & \Gamma^{n, k}, \rho \vdash i: \text { int } \\
\text { 6, tautology } & \Gamma^{n, k}, \rho \vdash i \leq i \text { true } \\
\text { 7, def. of subs. } & \Gamma^{n, k}, \rho \vdash(y \leq i)\{i / y\} \text { true } \\
\text { 6,8, index form. } & \Gamma^{n, k}, \rho \vdash i:\{y: \text { int } \mid y \leq i\} \\
\text { 3,9, lemma D.29 } & \Gamma^{n, k}, \rho \vdash e\{i / x\}: T\{i / x\} \\
\text { 3, agreement } & \Gamma^{n, k}, \rho, x:\{y: \text { int } \mid y \leq i\}: \text { context } \\
\text { 11, subtyping } & \Gamma^{n, k}, \rho \vdash\{y: \text { int } \mid y \leq i-1\}<:\{y: \text { int } \mid y \leq i\} \\
\text { 3,12, lemma D.28 } & \Gamma^{n, k}, \rho, x:\{y: \text { int } \mid y \leq i-1\} \vdash e: T \\
\text { 13, exp. formation } & \Gamma^{n, k}, \rho \vdash \text { for } x: i-1 . .1 \text { do } e: \forall x \leq i-1 . T \\
\text { 10,14, exp. formation } & \Gamma^{n, k}, \rho \vdash(e\{i / x\} ; \text { for } x: i-1 . .1 \text { do } e): \\
& \\
\text { 3, lemma D.25 } & \Gamma^{n, k}, \rho, x:\{y: \text { int } \mid y \leq i\} \vdash T: \text { type } \\
\text { 2, 16, type eq. } & \Gamma^{n, k}, \rho \vdash(T\{i / x\} ; \forall x \leq i-1 . T) \equiv \forall x \leq i . T \\
\text { 10,17, exp.+proc. form. } & \Gamma^{n, k} \vdash(\rho, e\{i / x\} ; \text { for } x: i-1 . .1 \text { do } e): \forall x \leq i . T
\end{array}
$$

\section{Lemma 4.3 (progress for processes). Statement on page 9.}

Proof. By analysis of the hypotheses, with one case for each rule, and a special treatment for $\Gamma^{n, k}, \rho \vdash e$ : skip.

Case let rule: Building from $\Gamma^{n, k}, \rho \vdash i: D$ and the fact that evaluation succeeds (Lemma D.39), we obtain $(\rho, i) \downarrow^{n, k}$ $\left(\rho^{\prime}, v\right): D$. This, combined with premises $\Gamma^{n, k}, \rho, x: D \vdash e: T$ and $x \notin \mathrm{fv}(T)$ constitute the necessary conditions to apply reduction for let processes in Figure 25, obtaining $(\rho$, let $x: D=i$ in $e) \rightarrow^{n, k}\left(\rho^{\prime}, e\{v / x\}\right)$.

Case if $p$ then $e_{1}$ else $e_{2}$, while $p$ do $e$, and for $x: i . .1$ do $e$ rules: Similar to let.

Case $\Gamma^{n, k}, \rho \vdash e$ : skip: By induction on this assertion, using the inversion lemma for expression formation (Lemma D.31).

Subcase $e$ is skip: we are done.

Subcase $e$ is while $p$ do $e_{1}$ and $\Gamma^{n, k}, \rho \vdash p:$ prop and $\Gamma^{n, k}, \rho, p \vdash e_{1}$ : skip. Analysing the possible truth values of proposition $p$, we know that either formulae $\left(\Gamma^{n, k}, \rho\right) \models p$ or formulae $\left(\Gamma^{n, k}, \rho\right) \not \models p$. We show the first case (the other is similar). If formulae $\left(\Gamma^{n, k}, \rho\right) \models p$ and $\Gamma^{n, k}, \rho \vdash p$ : prop then $\Gamma^{n, k}, \rho \vdash p$ true. By applying context exchange for expressions (Lemma D.30) followed by process reduction rules we conclude that $\left(\rho\right.$, while $p$ do $\left.e_{1}\right) \rightarrow^{n, k}\left(\rho, e_{1}\right.$; while $p$ do $\left.e_{1}\right)$.

Subcase $e$ is for $x: i . .1$ do $e_{1}, \Gamma^{n, k}, \rho \vdash i<1$ true and $\Gamma^{n, k}, \rho, x:\{y:$ int $\mid y \leq i\} \vdash e_{1}$ : skip. By application of context exchange for expressions (Lemma D.30) followed by process reduction rules we can conclude that ( $\rho$, for $x: i . .1$ do $\left.e_{1}\right) \rightarrow^{n, k}$ 
$(\rho$, skip $)$.

Subcase $e$ is let $y: D_{1}=i_{1}$ in $e_{1}$, and (1) $\Gamma^{n, k}, \rho \vdash i_{1}: D_{1}$ and (2) $y \notin \mathrm{fv}(\mathrm{skip})$, and (3) $\Gamma^{n, k}, \rho, y: D_{1} \vdash e_{1}:$ skip: By applying evaluation always succeeds (lemma D.39) to (1), we derive that (4) $(\rho, i) \downarrow^{n, k}\left(\rho^{\prime}, v\right): D$. By applying (2-4) and process reduction rules, we can conclude that $\left(\rho\right.$, let $y: D_{1}=i_{1}$ in $\left.e_{1}\right) \rightarrow^{n, k}\left(\rho^{\prime}, e_{1}\{v / y\}\right)$.

Subcase $e$ is if $p$ then $e_{1}$ else $e_{2}$, and $\Gamma^{n, k} \vdash p:$ prop, and $\Gamma^{n, k}, \rho, p \vdash e_{1}:$ skip, and $\Gamma^{n, k}, \rho, \neg p \vdash e_{2}:$ skip: Similar to while.

Subcase $e$ is $e_{1} ; e_{2}$, and (1) $\Gamma^{n, k}, \rho \vdash e_{1}$ : skip, and (2) $\Gamma^{n, k}, \rho \vdash e_{2}$ : skip. By induction on (1) we obtain $e_{1}$ is skip or $\left(\rho, e_{1}\right) \rightarrow^{n, k} q$. It is easy to show that for both cases there is a process reduction rule such that $\left(\rho, e_{1} ; e_{2}\right) \rightarrow^{n, k} q$.

\section{D.8 Results related to programs}

Main results in this section: agreement for program formation (Lemma D.41), agreement for program reduction (Theorem 4.4), and progress for programs (Theorem 4.6).

Lemma D.41 (agreement for program formation).

$$
\frac{P: S}{S: \text { ptype }}
$$

Proof. Directly from the premise of the only rule for assertion $P: S$.

Theorem 4.4 (agreement for program reduction). Statement on page 10.

Proof. By case analysis on the rules concluding the hypothesis. We have eight cases: one dealing with message passing, one dealing with process reductions and six dealing with collective operations. We illustrate one case for each category.

Case the derivation ends with the broadcast rule:

$$
\begin{array}{ll}
\text { rule premise } & \left(\rho_{l}, i_{l}^{\prime}\right) \downarrow^{n, l}\left(\rho_{l}^{\prime}, v\right): D \\
\text { rule premise } & \Gamma^{n} \vdash 1 \leq i_{k} \leq n \text { true } \\
\text { rule premise } & \Gamma^{n, k}, \rho_{k} \vdash i_{k}^{\prime}: D \\
\text { rule premise } & \Gamma^{n, k}, x: D, \rho_{k} \vdash e_{k}: T \\
\text { rule premise } & D, T \text { contain no ref types } \\
\text { rule premise } & \text { rank } \notin \mathrm{fv}(D, T)
\end{array}
$$

Building the first result, $P_{1}: S_{1}$ :

2-4, 6, lemma D.2, exp.+proc. form.

$$
\begin{aligned}
& \Gamma^{n, k} \vdash\left(\rho_{k}, \text { let } x: D=\text { broadcast } i_{k} i_{k}^{\prime} \text { in } e_{k}\right): \text { broadcast } i_{k} x: D . T \\
& \Gamma^{n}, x: D \vdash T: \text { type } \\
& \Gamma^{n} \vdash \text { broadcast } i_{k} x: D . T \equiv \text { broadcast } i_{k} x: D . T: \text { type } \\
& \left(\text { broadcast } i_{k} x: D \cdot T\right)_{k=1}^{n}: \text { ptype } \\
& \left(\rho_{k}, \text { let } x: D=\text { broadcast } i_{k} i_{k}^{\prime} \text { in } e_{k}\right)_{n=1}^{n}:\left(\text { broadcast } i_{k} x: D \cdot T\right)_{k=1}^{n}
\end{aligned}
$$

Building the second result, $P_{2}: S_{2}$ :

$$
\begin{aligned}
& \text { 2, lemma D.37 } \\
& \text { 2, lemma D.37 } \\
& \text { 2, lemma D.37 } \\
& \text { 2, 4, 6, lemma D.3 }
\end{aligned}
$$

$$
\begin{aligned}
& \Gamma^{n, l}, \rho_{l} \vdash i_{l}^{\prime}: D \\
& \rho_{l}^{\prime} \vdash v: D \\
& \rho_{l}^{\prime}=\rho_{l}, \rho_{l}^{\prime \prime} \\
& \varepsilon \vdash v: D
\end{aligned}
$$

rank $\neq l:$

$$
\begin{aligned}
& 15, \text { lemma D. } 2 \\
& 5,8, \text { lemma D.29 }
\end{aligned}
$$

$$
\begin{aligned}
& \Gamma^{n, k}, \rho_{k} \vdash v: D \\
& \Gamma^{n, k}, \rho_{k} \vdash e\{v / x\}: T\{v / x\}
\end{aligned}
$$


15 , proc. formation

$\Gamma^{n, k}, \rho_{k} \vdash\left(\rho_{k}, e\{v / x\}\right): T\{v / x\}$

rank $=l$ :

5, 14, lemma D.2

as for rank $\neq l$

15 , lemma D.2

8,21 , lemma D.7, ref. type eq.

22 , program type formation

$18,20,22$, proc+proc. form.
$\Gamma^{n, l}, \rho_{l}^{\prime} \vdash e_{l}: T$

$\Gamma^{n, l}, \rho_{l}^{\prime} \vdash\left(\rho_{l}, e\{v / x\}\right): T\{v / x\}$

$\Gamma^{n}, \rho_{k} \vdash v: D$

$\Gamma^{n} \vdash T\{v / x\} \equiv T\{v / x\}:$ type $\quad(1 \leq k, l \leq n)$

$(T\{v / x\})_{k=1}^{n}:$ ptype

$\left(\rho_{k}, e_{k}\{v / x\}\right)_{k=1}^{l-1},\left(\rho_{l}^{\prime}, e_{l}\{v / x\}\right),\left(\rho_{k}, e_{k}\{v / x\}\right)_{k=l+1}^{n}:(T\{v / x\})_{k=1}^{n}$

Case the derivation ends with the message rule:

$\begin{array}{ll}\text { shape of the rule } & 1 \leq l, m \leq n \\ \text { rule premise } & i_{l} \downarrow^{n} m \\ \text { rule premise } & \left(\rho_{l}, i_{l}^{\prime}\right) \downarrow^{n, l}\left(\rho_{l}^{\prime}, v\right): D \\ \text { rule premise } & i_{m} \downarrow^{n} l \\ \text { rule premise } & \left(\rho_{m}, i_{m}^{\prime}\right) \downarrow^{n, m}\left(\rho_{m}^{\prime}, r\right): D \text { ref } \\ \text { rule premise } & \Gamma \vdash l \neq m \text { true } \\ \text { rule premise } & \Gamma^{n, l} \vdash e_{l}: T \\ \text { rule premise } & \Gamma^{n, m} \vdash e_{m}: T \\ \text { rule premise } & \Gamma^{n, k} \vdash q_{k}: T \quad(k=1 . . n, k \neq l, m) \\ \text { rule premise } & D, T \operatorname{contain} \text { no ref types } \\ \text { rule premise } & \text { rank } \notin \mathrm{fv}(D, T)\end{array}$

We proceed by building the first result, namely $P_{1}: S_{1}$. The types for processes $\left(\rho_{l}\right.$, send $\left.i_{l} i_{l}^{\prime}\right)$ and $\left(\rho_{m}\right.$, receive $\left.i_{m} i_{m}^{\prime}\right)$ follow from Lemma D.38 and from the constraints imposed on $l$ and $m$ :

$$
\begin{array}{ll}
\text { 2, lemma D.38 } & \Gamma^{n} \vdash i_{l}=m \text { true } \\
\text { 3, lemma D.37 } & \Gamma^{n, l}, \rho_{l}^{\prime} \vdash i_{l}^{\prime}: D \\
1,12 \text {, transitivity } & \Gamma^{n} \vdash 1 \leq i_{l} \leq \text { size true }
\end{array}
$$

From the definition of $\Gamma^{n, l}$, we know that $\Gamma^{n, l} \vdash$ rank $=l$ true.

$$
6, \Gamma^{n, l} \vdash \text { rank }=l \text { true } \quad \Gamma^{n, l} \vdash m \neq \text { rank true }
$$

Weakening (Lemma D.2) is used in rules (13-15) to extend the type environment to $\Gamma^{n, l}, \rho_{l}^{\prime}$ (details omitted). Such steps build the type for send:

$$
\begin{array}{ll}
\text { 13, 14, 15, lemma D.2, exp. form. } & \Gamma^{n, l}, \rho_{l}^{\prime} \vdash \text { send } i_{l} i_{l}^{\prime} \text { : message } l i_{l} D \\
\text { 16, 10, lemma D.27 } & \Gamma^{n, l}, \rho_{l} \vdash \text { send } i_{l} i_{l}^{\prime}: \text { message } l i_{l} D \\
\text { 7, lemma D.2 } & \Gamma^{n, l}, \rho_{l} \vdash e_{l}: T \\
17,18, \text { exp. form. } & \Gamma^{n, l}, \rho_{l} \vdash \text { send } i_{l} i_{l}^{\prime} ; e_{l}: \text { message } l i_{l} D ; T
\end{array}
$$

A similar derivation yields the type for receive:

$$
\begin{array}{ll}
\text { 4, lemma D.38 } & \Gamma^{n} \vdash i_{m}=l \text { true } \\
1,20, \text { transitivity } & \Gamma^{n} \vdash 1 \leq i_{m} \leq \text { size true } \\
5 \text {, lemma D.37 } & \Gamma^{n, m}, \rho_{m}^{\prime} \vdash i_{m}^{\prime}: D \text { ref }
\end{array}
$$


Def. of $\Gamma^{n, m}$

6,23 , transitivity

$21,22,24$, lemma D.2, exp. form.

25, 10, lemma D.27

8, lemma D.2

26, 27, exp. form.

$$
\begin{aligned}
& \Gamma^{n, m} \vdash \text { rank }=m \text { true } \\
& \Gamma^{n, m} \vdash \text { rank } \neq l \text { true } \\
& \Gamma^{n, m}, \rho_{m}^{\prime} \vdash \text { receive } i_{m} i_{m}^{\prime}: \text { message } i_{m} m D \\
& \Gamma^{n, m}, \rho_{m} \vdash \text { receive } i_{m} i_{m}^{\prime}: \text { message } i_{m} m D \\
& \Gamma^{n, m}, \rho_{m} \vdash e_{m}: T \\
& \Gamma^{n, m}, \rho_{m} \vdash \text { receive } i_{m} i_{m}^{\prime}: \text { message } i_{m} m D ; T
\end{aligned}
$$

Building the ptype:

$$
\begin{array}{ll}
12,19, \text { proc. form. } & \Gamma^{n, l} \vdash\left(\rho_{l}, \text { send } m i_{l}^{\prime} ; e_{l}\right): \text { message } l m D ; T \\
20,28, \text { proc. form. } & \Gamma^{n, m} \vdash\left(\rho_{m}, \text { receive } l i_{m}^{\prime} ; e_{m}\right): \text { message } l m D ; T
\end{array}
$$

Notice that from type equality, message types who do not have rank as a sender or receiver are equated to skip. We use such fact to build a common type for the vector.

$$
\begin{aligned}
& 1,6,9, \text { transitivity } \\
& \Gamma^{n, k} \vdash l, m \neq \text { rank true } \quad(k=1 . . n, k \neq l, m) \\
& 1,6 \text {, def. of } \Gamma^{n}, \text { lemma D.2 } \\
& \Gamma^{n, k} \vdash 1 \leq l, m \leq \text { size } \wedge l \neq m \text { true } \\
& \text { 13, lemma D.1 } \\
& \Gamma^{n, l}, \rho_{l}^{\prime} \vdash D: \text { dtype } \\
& \text { 33, 10, 11, lemma D. } 3 \\
& \Gamma^{n} \vdash D: \text { dtype } \\
& \text { 34, lemma D.2 } \\
& \Gamma^{n, k} \vdash D \text { : dtype } \\
& 31,32,35 \text {, 三 form. } \\
& \Gamma^{n, k} \vdash \text { message } l m D \equiv \text { skip } \\
& 9 \text {, inversion } \\
& \Gamma^{n, k}, \rho_{k} \vdash e_{k}: T \\
& \Gamma^{n, k}, \rho_{k} \vdash T: \text { type } \\
& \Gamma^{n, k}, \rho_{k} \vdash \text { skip } T \text { E message } l m D ; T \\
& \Gamma^{n, k} \vdash\left(\rho_{k}, e_{k}\right) \text { : message } l m D ; T
\end{aligned}
$$

Putting all together:

$$
29,30,40 \text {, proc. inversion, lem. D.25 } \quad \Gamma^{n, k} \vdash T_{k} \equiv \text { message } l m D ; T: \text { type }
$$

41, ptype formation

$7,29,30,41$, prog. form.

$$
\text { (message } l \mathrm{~m} D ; T)_{k=1}^{n} \text { : ptype }
$$$$
\left(q_{k}\right)_{k=1}^{l-1},\left(\rho_{l} \text {, send } i_{l} i_{l}^{\prime} ; e_{l}\right),\left(q_{k}\right)_{k=l+1}^{m-1},\left(\rho_{m} \text {, receive } i_{m} i_{m}^{\prime} ; e_{m}\right),\left(q_{k}\right)_{k=m+1}^{n}
$$$$
\text { : (message } l \mathrm{~m} D ; T)_{k=1}^{n}
$$

Building the second result, $P_{2}: S_{2}$ :

$$
\begin{array}{ll}
\text { 3, lemma D.37 } & \Gamma^{n, l}, \rho_{l}^{\prime} \vdash v: D \\
\text { 10, 11, 44, lemma D.3 } & \Gamma^{n} \vdash v: D \\
\text { 45, lemma D.2 } & \Gamma^{n, m}, \rho_{m}^{\prime} \vdash v: D \\
\text { 5, lemma D.37 } & \Gamma^{n, m}, \rho_{m}^{\prime} \vdash r: D \text { ref } \\
\text { 46, 47, store update } & \Gamma^{n, m}, \rho_{m}^{\prime}[r:=v] \vdash r: D \text { ref } \\
\text { 48, lemma D.22 - applied twice } & \Gamma^{n, m}, \rho_{m}^{\prime}[r:=v]: \text { context } \\
\text { 8, 49, lemma D.26 } & \Gamma^{n, m}, \rho_{m}^{\prime}[r:=v] \vdash e_{m}: T \\
\text { 50, proc. form } & \Gamma^{n, m} \vdash\left(\rho_{m}^{\prime}[r:=v], e_{m}\right): T \\
\text { 44, lemma D.22 - applied twice } & \Gamma^{n, l}, \rho_{l}^{\prime}: \text { context } \\
\text { 7, 52, lemma D.26 } & \Gamma^{n, l}, \rho_{l}^{\prime} \vdash e_{l}: T \\
\text { 53, proc. form } & \Gamma^{n, l} \vdash\left(\rho_{l}^{\prime}, e_{l}\right): T
\end{array}
$$

Joining (9), (51) and (54), we can see that the continuations have the same type:

$$
9,10 \text {, inversion, lemmas D.22, D.3 } \quad \Gamma^{n, k} \vdash T: \text { type } \quad(k=1 . . n, k \neq l, m)
$$


51, 10, inversion, lemmas D.22, D.3 $\quad \Gamma^{n, l} \vdash T$ : type

54,10 , inversion, lemmas D.22, D.3 $\quad \Gamma^{n, m} \vdash T:$ type

$55,56,57, \equiv$ form.

$\Gamma^{n, k} \vdash T_{k} \equiv T \quad(k=1 . . n, k)$

58 , ptype formation

$(T)_{k=1}^{n}:$ ptype

$9,51,54,59$, prog. formation

$\left(q_{k}\right)_{k=1}^{l-1},\left(\rho_{l}^{\prime}, e_{l}\right),\left(q_{k}\right)_{k=l+1}^{m-1},\left(\rho_{m}^{\prime}[r:=v], e_{m}\right),\left(q_{k}\right)_{k=m+1}^{n}:(T)_{k=1}^{n}$

Case the derivation ends with the scatter rule:

$\begin{array}{ll}\text { rule premise } & i_{k} \downarrow^{n} l \\ \text { rule premise } & \left(\rho_{k}, i_{k}^{\prime}\right) \downarrow^{n, k}\left(\rho_{k}^{\prime}, r_{k}\right): \text { float array ref } \quad(k=1 . . n) \\ \text { rule premise } & \left(\rho_{l}^{\prime}, i_{l}^{\prime \prime}\right) \downarrow^{n, l}\left(\rho_{l}^{\prime \prime},\left[\vec{v}_{1}, \ldots, \vec{v}_{n}\right]\right):\left\{x: \text { float array } \mid \text { len }(x)=\operatorname{size} * \operatorname{len}\left(i_{k}^{\prime}\right)\right\} \\ \text { rule premise } & \Gamma^{n, k}, \rho_{k},\left\{\text { rank }=i_{k}\right\} \vdash i_{k}^{\prime \prime}:\left\{x: \text { float array } \mid \text { len }(x)=\operatorname{size} * \operatorname{len}\left(i_{k}^{\prime}\right)\right\} \\ \text { rule premise } & D, T \text { contain no ref types } \\ \text { rule premise } & \text { rank } \notin \mathrm{fv}(D, T)\end{array}$

We now proceed by proving the left-hand side, namely: $\left(\rho_{k} \text {, scatter } i_{k} i_{k}^{\prime} i_{k}^{\prime \prime}\right)_{k=1}^{n}: S_{1}$.

$\begin{array}{ll}\text { 1, lem. D.38 } & \Gamma^{n} \vdash i_{k}=l \text { true } \quad(k=1 . . n) \\ \text { 2, lem. D.37 } & \rho_{k} \text { to } \Gamma_{k} \\ \text { 2, lem. D.37 } & \rho_{k}^{\prime} \text { to } \Gamma_{k}^{\prime} \\ \text { 2, lem. D.37 } & \Gamma_{k}^{\prime} \text { is } \Gamma_{k}, \Gamma_{k}^{\prime \prime} \\ \text { 2, lem. D.37 } & \Gamma^{n, l}, \Gamma_{k}^{\prime} \vdash i_{k}^{\prime}: \text { float array ref } \\ \text { 2, lem. D.37 } & \Gamma_{k}^{\prime} \vdash r_{k}: \text { float array ref } \\ \text { 3, lem. D.37 } & \rho_{l}^{\prime} \text { to } \Gamma_{l}^{\prime} \\ \text { 3, lem. D.37 } & \rho_{l}^{\prime \prime} t o \Gamma_{l}^{\prime \prime} \\ \text { 3, lem. D.37 } & \Gamma_{l}^{\prime \prime} \text { is } \Gamma_{l}^{\prime}, \Gamma_{l}^{\prime \prime \prime} \\ \text { 3, lem. D.37 } & \Gamma^{n, l}, \Gamma_{l}^{\prime \prime} \vdash i_{l}^{\prime \prime}:\left\{x: \text { float array } \mid \operatorname{len}(x)=\operatorname{size} * \operatorname{len}\left(i_{k}^{\prime}\right)\right\} \\ \text { 3, lem. D.37 } & \Gamma_{l}^{\prime \prime} \vdash\left[\vec{v}_{1}, \ldots, \vec{v}_{n}\right]:\left\{x: \text { float array } \mid \operatorname{len}(x)=\operatorname{size} * \operatorname{len}\left(i_{k}^{\prime}\right)\right\}\end{array}$

From the structure of the program reduction rule, we know that $\cdot \vdash 1 \leq l \leq$ size true, then:

8, lemma D.32

$\Gamma_{k}:$ context

$7,18, \cdot \vdash 1 \leq l \leq$ size true, lem. D. $2 \quad \Gamma^{n, k} \vdash 1 \leq i_{k} \leq$ size true

$4,11,19$, exp. form.

$\Gamma^{n, k}, \Gamma_{k} \vdash$ scatter $i_{k} i_{k}^{\prime} i_{k}^{\prime \prime}:$ scatter $i_{k}\left\{x:\right.$ float array $\left.\mid \operatorname{len}(x)=\operatorname{size} * \operatorname{len}\left(i_{k}^{\prime}\right)\right\}$

20, proc. form.

$\Gamma^{n, k} \vdash\left(\rho_{k}, \text { scatter } i_{k} i_{k}^{\prime} i_{k}^{\prime \prime}\right)_{k=1}^{n}:$ scatter $i_{k}\left\{x:\right.$ float array $\left.\mid \operatorname{len}(x)=\operatorname{size} * \operatorname{len}\left(i_{k}^{\prime}\right)\right\}$

We continue by proving $\Gamma^{n, k} \vdash$ scatter $i_{k}\left\{x:\right.$ float array $\left.\mid \operatorname{len}(x)=\operatorname{size} * \operatorname{len}\left(i_{k}^{\prime}\right)\right\} \equiv T: \operatorname{type:}$

21, lemma D.40 $\quad \Gamma^{n, k} \vdash$ scatter $i_{k}\left\{x:\right.$ float array $\left.\mid \operatorname{len}(x)=\operatorname{size} * \operatorname{len}\left(i_{k}^{\prime}\right)\right\}:$ type

22, lem. D.11 $\quad \Gamma^{n, k} \vdash$ scatter $i_{k}\left\{x:\right.$ float array $\left.\mid \operatorname{len}(x)=\operatorname{size} * \operatorname{len}\left(i_{k}^{\prime}\right)\right\} \equiv \operatorname{scatter} i_{k}\left\{x:\right.$ float array $\left.\mid \operatorname{len}(x)=\operatorname{size} * \operatorname{len}\left(i_{k}^{\prime}\right)\right\}$

23, ptype form. $\quad\left(\text { scatter } i_{k}\left(\left\{x: D \text { array } \mid \operatorname{len}(x) * \operatorname{size}=\operatorname{len}\left(i_{k}^{\prime \prime}\right)\right\}\right)\right)_{k=1}^{n}:$ ptype

Joining (21) and (24), we can derive the program type:

21, 24, prog. formation $\quad\left(\rho_{k}, \text { scatter } i_{k} i_{k}^{\prime} i_{k}^{\prime \prime}\right)_{k=1}^{n}:\left(\operatorname{scatter} i_{k}\left(\left\{x: D \text { array } \mid \operatorname{len}(x) * \operatorname{size}=\operatorname{len}\left(i_{k}^{\prime \prime}\right)\right\}\right)\right)_{k=1}^{n}$ 

$S_{2}$.

We continue by proving the right-hand side, namely: $\left(\rho_{k}^{\prime}\left[r_{k}:=\left[\vec{v}_{k}\right]\right], \text { skip }\right)_{k=1}^{l-1},\left(\rho_{l}^{\prime \prime}\left[r_{l}:=\left[\vec{v}_{l}\right]\right]\right.$, skip $),\left(\rho_{k}^{\prime}\left[r_{k}:=\left[\vec{v}_{k}\right]\right], \text { skip }\right)_{k=l+1}^{n}:$

$$
\begin{aligned}
& \Gamma^{n}, \Gamma_{k}^{\prime} \vdash r_{k}: \text { float array ref } \\
& \Gamma_{l}^{\prime \prime} \vdash\left[\vec{v}_{1}, \ldots \vec{v}_{n}\right]: \text { float array } \\
& \Gamma_{l}^{\prime \prime} \vdash\left[\vec{v}_{k}\right]: \text { float }
\end{aligned}
$$

In order to prove $\rho_{k}^{\prime}\left[r_{k}:=\left[\overrightarrow{v_{1}}, \ldots \overrightarrow{v_{n}}\right]\right]$ to $\Gamma_{k}^{\prime}$ we need to ensure that: (A) $\rho_{k}^{\prime}$ to $\Gamma_{k}^{\prime}$, (B) $\Gamma^{n}, \Gamma_{k}^{\prime} \vdash r_{k}: D$ ref and (C) $\Gamma^{n}, \Gamma_{k}^{\prime} \vdash\left[\vec{v}_{k}\right]: D$. We have already (A) from eq. 9 , and applying weakening and context exchange to eq. 12 we can derive that $\Gamma^{n}, \Gamma_{k}^{\prime} \vdash r_{k}$ : float array ref. How do we use eq. 28 to derive (C)?

Case the derivation ends with the gather rule:

$$
\begin{array}{ll}
\text { rule premise } & i_{k} \downarrow^{n} l \\
\text { rule premise } & \left(\rho_{k}, i_{k}^{\prime}\right) \downarrow^{n, k}\left(\rho_{k}^{\prime},\left[\vec{v}_{k}\right]\right): \text { float array } \quad(k=1 . . n) \\
\text { rule premise } & \left(\rho_{l}^{\prime}, i_{l}^{\prime \prime}\right) \downarrow^{n, l}\left(\rho_{l}^{\prime \prime}, r\right):\left\{x: \text { float array } \mid \operatorname{len}(x)=\operatorname{size} * \operatorname{len}\left(i_{k}^{\prime}\right)\right\} \text { ref } \\
\text { rule premise } & \Gamma^{n, k}, \rho_{k},\left\{\text { rank }=i_{k}\right\} \vdash i_{k}^{\prime \prime}:\left\{x: \text { float array } \mid \text { len }(x)=\operatorname{size} * \operatorname{len}\left(i_{k}^{\prime}\right)\right\} \\
\text { rule premise } & D, T \text { contain no ref types } \\
\text { rule premise } & \text { rank } \notin \mathrm{fv}(D, T)
\end{array}
$$

We start by proving the left hand side, $\left(\rho_{k} \text {, gather } i_{k} i_{k}^{\prime} i_{k}^{\prime \prime}\right)_{k=1}^{n}: S_{1}$.

$$
\begin{array}{ll}
\text { 1, lem. D.38 } & \Gamma^{n} \vdash i_{k}=l \text { true } \\
\text { 2, lem. D.37 } & \rho_{k} \text { to } \Gamma_{k} \\
\text { 2, lem. D.37 } & \rho_{k}^{\prime} \text { to } \Gamma_{k}^{\prime} \\
\text { 2, lem. D.37 } & \Gamma_{k}^{\prime} \text { is } \Gamma_{k}, \Gamma_{k}^{\prime \prime} \\
\text { 2, lem. D.37 } & \Gamma^{n, k}, \Gamma_{k}^{\prime} \vdash i_{k}^{\prime}: \text { float array } \\
\text { 2, lem. D.37 } & \Gamma_{k}^{\prime} \vdash\left[\vec{v}_{k}\right]: \text { float array } \\
\text { 3, lem. D.37 } & \rho_{l}^{\prime} \text { to } \Gamma_{l} \\
\text { 3, lem. D.37 } & \rho_{l}^{\prime \prime} \text { to } \Gamma_{l}^{\prime \prime} \\
\text { 3, lem. D.37 } & \Gamma_{l}^{\prime \prime} \text { is } \Gamma_{l}^{\prime}, \Gamma_{l}^{\prime \prime \prime} \\
\text { 3, lem. D.37 } & \Gamma^{n, l}, \Gamma_{l}^{\prime \prime} \vdash i_{l}^{\prime \prime}:\left\{x: \text { float array } \mid \operatorname{len}(x)=\operatorname{size} * \operatorname{len}\left(i_{k}^{\prime}\right)\right\} \text { ref } \\
\text { 3, lem. D.37 } & \Gamma_{l}^{\prime \prime} \vdash r:\left\{x: \text { float array } \mid \operatorname{len}(x)=\operatorname{size} * \operatorname{len}\left(i_{k}^{\prime}\right)\right\} \text { ref }
\end{array}
$$

From the structure of the program reduction rule we can infer that $\cdot \vdash 1 \leq l \leq$ size true, then:

$7, \vdash 1 \leq l \leq$ size true $\quad \Gamma^{n} \vdash 1 \leq i_{k} \leq$ size true

8, lem. D.1 $\quad \Gamma_{k}:$ context

18, 19, lem. D.2 $\quad \Gamma^{n, k} \vdash 1 \leq i_{k} \leq$ size true

18, 19, lem. D.3 $\quad \Gamma^{n, k}, \Gamma_{k} \vdash i_{k}^{\prime}$ : float array

4, 20, 21, exp. form. $\quad \Gamma^{n, k}, \Gamma_{k} \vdash$ gather $i_{k} i_{k}^{\prime} i_{k}^{\prime \prime}:$ gather $i_{k}\left\{x:\right.$ float array $\left.\mid \operatorname{len}(x)=\operatorname{size} * \operatorname{len}\left(i_{k}^{\prime}\right)\right\}$

22, proc. form. $\Gamma^{n, k} \vdash\left(\rho_{k}\right.$, gather $\left.i_{k} i_{k}^{\prime} i_{k}^{\prime \prime}\right):$ gather $i_{k}\left\{x:\right.$ float array $\left.\mid \operatorname{len}(x)=\operatorname{size} * \operatorname{len}\left(i_{k}^{\prime}\right)\right\}$

23, lem. D.40 $\Gamma^{n, k} \vdash$ gather $i_{k}\left\{x:\right.$ float array $\left.\mid \operatorname{len}(x)=\operatorname{size} * \operatorname{len}\left(i_{k}^{\prime}\right)\right\}:$ type

24, lem. D.11 $\Gamma^{n, k} \vdash$ gather $i_{k}\left\{x:\right.$ float array $\left.\mid \operatorname{len}(x)=\operatorname{size} * \operatorname{len}\left(i_{k}^{\prime}\right)\right\} \equiv$ gather $i_{k}\left\{x:\right.$ float array $\left.\mid \operatorname{len}(x)=\operatorname{size} * \operatorname{len}\left(i_{k}^{\prime}\right)\right\}$

25, ptype form. (gather $i_{k}\left\{x:\right.$ float array $\left.\left.\mid \operatorname{len}(x)=\operatorname{size} * \operatorname{len}\left(i_{k}^{\prime}\right)\right\}\right)_{k=1}^{n}:$ ptype 
23, 26, prog. form. $\quad\left(\rho_{k}, \text { gather } i_{k} i_{k}^{\prime} i_{k}^{\prime \prime}\right)_{k=1}^{n}:\left(\text { gather } i_{k}\left\{x: \text { float array } \mid \operatorname{len}(x)=\operatorname{size} * \operatorname{len}\left(i_{k}^{\prime}\right)\right\}\right)_{k=1}^{n}$

We continue by proving the right hand side, namely $\left(\rho_{k}^{\prime} \text {, skip }\right)_{k=1}^{l-1},\left(\rho_{l}^{\prime \prime}\left[r:=\left[\vec{v}_{1}, \ldots, \vec{v}_{n}\right]\right]\right.$, skip $),\left(\rho_{k}^{\prime}, \text { skip }\right)_{k=l+1}^{n}: S_{2}$.

$\begin{array}{ll}\text { 11, lem. D.1 } & \Gamma^{n, k}, \Gamma_{k}^{\prime}: \text { context } \\ \text { 28, exp. form. } & \Gamma^{n, k}, \Gamma_{k}^{\prime} \vdash \text { skip : skip } \\ \text { 9, 29, proc. form. } & \Gamma^{n, k} \vdash\left(\rho_{k}^{\prime} \text {, skip }\right) \text { skip } \\ \text { 30, lem. D.40 } & \Gamma^{n, k} \vdash \text { skip : type } \\ \text { 31, lem. D.11 } & \Gamma^{n, k} \vdash \text { skip } \equiv \text { skip } \\ \text { 17, lem. D.2, lem. D.8 } & \Gamma^{n}, \Gamma_{l}^{\prime \prime} \vdash r:\left\{x: \text { float array } \mid \operatorname{len}(x)=\operatorname{size} * \operatorname{len}\left(i_{k}^{\prime}\right)\right\} \text { ref } \\ \ldots & \end{array}$

In order to establish that $\Gamma^{n, l} \vdash\left(\rho_{l}^{\prime \prime}\left[r:=\left[\vec{v}_{1}, \ldots, \vec{v}_{n}\right]\right]\right.$, skip) $: T_{l}$, we need to use lemma D.34 with premises $\rho_{l}^{\prime \prime}$ to $\Gamma_{l}^{\prime \prime}$ (eq. 14), $\Gamma^{n}, \Gamma_{l}^{\prime \prime} \vdash r:\left\{x:\right.$ float array $\left.\mid \operatorname{len}(x)=\operatorname{size} * \operatorname{len}\left(i_{k}^{\prime}\right)\right\}$ ref (eq. 33) and $\Gamma^{n}, \Gamma_{l}^{\prime \prime} \vdash\left[\vec{v}_{1}, \ldots, \vec{v}_{n}\right]:\left\{x:\right.$ float array $\mid$ len $\left.(x)=\operatorname{size} * \operatorname{len}\left(i_{k}^{\prime}\right)\right\}$. From (12) we know that

$$
\Gamma_{k}^{\prime} \vdash\left[\vec{v}_{k}\right]: \text { float array }
$$

, that can be transformed into $\Gamma^{n}, \Gamma_{l}^{\prime \prime} \vdash\left[\vec{v}_{k}\right]$ : float array by applying weakening and context exchange. However, I do not see how to prove the missing subtyping relation $\Gamma^{n}, \Gamma_{l}^{\prime \prime} \vdash$ float array $<:\left\{x:\right.$ float array $\mid \operatorname{len}(x)=\operatorname{size} *$ len $\left.\left(i_{k}^{\prime}\right)\right\}$. Is there a subtyping relation between them?

Case the derivation ends with process reduction:

$$
\begin{array}{ll}
\text { rule premise } & q_{l} \rightarrow^{n, l} q_{l}^{\prime} \\
\text { rule premise } & \Gamma^{n, k} \vdash q_{k}: T_{k} \\
\text { rule premise } & T_{1}, \ldots, T_{l}, \ldots, T_{n}: \text { ptype }
\end{array}
$$

The proof relies on agreement for process reduction (lemma 4.1).

$$
\begin{array}{ll}
2,3 \text {, prog. form. } & q_{1}, \ldots, q_{l}, \ldots, q_{n}: T_{1}, \ldots, T_{l}, \ldots, T_{n} \\
1, \text { lemma } 4.1 & \Gamma^{n, l} \vdash q_{l}^{\prime}: T_{l} \\
2,4,5, \text { prog. form. } & q_{1}, \ldots, q_{l}^{\prime}, \ldots, q_{n}: T_{1}, \ldots, T_{l}, \ldots, T_{n}
\end{array}
$$

Theorem 4.6 (progress for programs). Statement on page 10.

Proof. From the hypothesis and the formation rules for programs and processes we know that:

$$
\begin{aligned}
& P_{1} \text { is }\left(\rho_{1}, e_{1}\right), \ldots,\left(\rho_{n}, e_{n}\right) \\
& S_{1} \text { is } T_{1}, \ldots, T_{n} \\
& \Gamma^{n, k}, \rho_{k} \vdash e_{k}: T_{k} \quad(k=1 . . n) \\
& T_{1}, \ldots, T_{n}: \text { ptype }
\end{aligned}
$$

The proof proceeds by rule induction on assertion $T_{1}, \ldots, T_{n}:$ ptype. There are ten cases to consider. We illustrate a few. Case the derivation ends with the type equality rule:

rule premise

$$
T_{1}, \ldots, T_{k}^{\prime}, \ldots, T_{n}: \text { ptype }
$$


rule premise

3,6 , exp. formation

$1,3,7,5$, proc+prog. formation

8 , induction
$\Gamma^{n, k} \vdash T_{k} \equiv T_{k}^{\prime}:$ type

$\Gamma^{n, k}, \rho_{k} \vdash e_{k}: T_{k}^{\prime}$

$P_{1}: T_{1}, \ldots, T_{k}^{\prime}, \ldots, T_{n}$

$P+1$ halted or $P_{1} \rightarrow P_{2}$

Case the derivation ends with the broadcast rule:

$$
\begin{array}{ll}
\text { rule premise } & \Gamma^{n} \vdash 1 \leq l \leq n \text { true } \\
\text { rule premise } & \Gamma^{n}, x: D \vdash T: \text { type } \\
6, \text { lemma D.2, lemma D.8 } & \Gamma^{n, k}, \rho_{k}, x: D \vdash T: \text { type }
\end{array}
$$

Each expression $e_{k}(1 \leq k \leq n)$ may be of four different forms, according to inversion for expression formation: Subcase each expression $e_{k}$ is let $x: D_{1}=$ broadcast $i_{k} i_{k}^{\prime}$ in $e_{k}^{\prime}$ :

$\begin{array}{ll}\text { lemma D.31 } & \Gamma^{n, k}, \rho_{k} \vdash D_{1} \equiv D: \text { dtype } \\ \text { lemma D.31 } & \Gamma^{n, k}, \rho_{k} \vdash i_{k}=l \text { true } \\ \text { lemma D.31 } & \Gamma^{n, k}, \rho_{k} \vdash 1 \leq i_{k} \leq \text { size true } \\ \text { lemma D.31 } & \Gamma^{n, k}, \rho_{k} \vdash i_{k}^{\prime}: D \\ \text { lemma D.31 } & \text { rank } \notin \mathrm{fv}\left(i_{1}\right) \\ \text { lemma D.31 } & \Gamma^{n, k}, x: D_{1}, \rho_{k} \vdash e_{k}^{\prime}: T\end{array}$

Building from index typing, deducibility and the fact that evaluation always succeeds, we get:

$\begin{array}{ll}5 \text {, inversion } & \Gamma^{n} \vdash 1 \leq l \leq n: \text { prop } \\ \text { 14, index form. } & \Gamma^{n} \vdash l: \text { int } \\ \text { 14, 15, index form. } & \Gamma^{n} \vdash l:\{y: \text { int } \mid 1 \leq y \leq n\} \\ 9,12 \text {, inversion, lemmas D.24, D.3 } & \Gamma^{n} \vdash i_{k}: \text { int } \\ 17, \text { lemma D.39 } & i_{k} \downarrow^{n} l \\ 11, \text { lemma D.39 } & \left(\rho_{k}, i_{k}^{\prime}\right) \downarrow^{n, k}\left(\rho_{k}^{\prime \prime}, v\right): D\end{array}$

We have now all the ingredients to perform reduction:

$$
\begin{array}{ll}
10,11,13,18,19, \text { prog. red. } \quad & \left(\rho_{k}, \text { let } x: D=\text { broadcast } i_{k} i_{k}^{\prime} \text { in } e_{k}^{\prime}\right)_{k=1}^{n} \rightarrow \\
& \left(\rho_{k}, e_{k}^{\prime}\{v / x\}\right)_{k=1}^{i-1},\left(\rho_{i}^{\prime \prime}, e_{i}^{\prime}\{v / x\}\right),\left(\rho_{k}, e_{k}^{\prime}\{v / x\}\right)_{k=i+1}^{n}
\end{array}
$$

Subcase at least one of the expressions $e_{k}$ is not broadcast.

From inversion of expression formation we have three additional cases.

Subsubcase $e_{k}$ is let $y_{k}: D_{k}=i_{k}^{\prime}$ in $e_{k}^{\prime}$ :

$$
\begin{array}{ll}
\text { lemma D.31 } & \Gamma^{n, k}, \rho_{k} \vdash i_{k}^{\prime}: D_{k} \\
\text { lemma D.31 } & y \notin \mathrm{fv}(\text { broadcast } i x: D . T) \\
\text { lemma D.31 } & \Gamma^{n, k}, \rho_{k}, y_{k}: D_{k} \vdash e_{k}^{\prime}: \text { broadcast } i_{k} x: D . T
\end{array}
$$

We apply progress for processes followed by program reduction:

$$
\begin{aligned}
& 21,22,23, \text { lemma } 4.3 \\
& 3,4,24 \text {, prog. red. }
\end{aligned}
$$$$
\left(\rho_{k} \text {, let } y_{k}: D_{k}=i_{k}^{\prime} \text { in } e_{k}\right) \rightarrow^{n, k} q_{k}
$$$$
P_{1} \rightarrow\left(\rho_{1}, e_{1}\right), \ldots, q_{k}, \ldots,\left(\rho_{n}, e_{n}\right)
$$

Subsubcase $e_{k}$ is if $p$ then $e_{k}^{\prime}$ else $e_{k}^{\prime \prime}$ :

$$
\text { lemma D.31 }
$$

$$
\Gamma^{n, k}, \rho_{k} \vdash p: \text { prop }
$$




$$
\begin{array}{ll}
\text { lemma D.31 } & \Gamma^{n, k}, \rho_{k} \vdash e_{k}^{\prime}: \text { broadcast } i_{k} x: D . T \\
\text { lemma D.31 } & \Gamma^{n, k}, \rho_{k} \vdash e_{k}^{\prime \prime}: \text { broadcast } i_{k} x: D . T
\end{array}
$$

We just need to apply progress to processes and program reduction.

$$
\begin{array}{ll}
26,27,28, \text { lemma } 4.3 & \text { if } p \text { then } e_{k}^{\prime} \text { else } e_{k}^{\prime \prime} \rightarrow^{n, k} q_{k} \\
3,4,29, \text { prog. red. } & P_{1} \rightarrow\left(\rho_{1}, e_{1}\right), \ldots, q_{k}, \ldots,\left(\rho_{n}, e_{n}\right)
\end{array}
$$

Subsubcase $e_{k}$ is $e_{k}^{\prime} ; e_{k}^{\prime \prime}$ :

$$
\begin{aligned}
& \text { lemma D.31 } \\
& \text { lemma D.31 }
\end{aligned}
$$$$
\Gamma^{n, k}, \rho_{k} \vdash e_{k}^{\prime}: \text { skip }
$$$$
\Gamma^{n, k}, \rho_{k} \vdash e_{k}^{\prime \prime}: \text { broadcast } i_{k} x: D . T
$$

From progress for processes applied to $e_{k}^{\prime}$ we have:

$$
31 \text {, lemma } 4.3
$$

$$
e_{k}^{\prime} \text { is skip or }\left(\rho_{k}, e_{k}^{\prime}\right) \rightarrow^{n, k}\left(\rho_{k}^{\prime}, e_{k}^{\prime \prime \prime}\right)
$$

Subsubsubcase $e_{k}^{\prime}$ is skip: Follows by simple application of process and program reduction rules:

33

32,34 , proc. red.

$3,4,36$, prog. red.

$e_{k}^{\prime}$ is skip

$$
\begin{aligned}
& \left(\rho_{k}, \text { skip } ; e_{k}^{\prime \prime}\right) \rightarrow^{n, k}\left(\rho_{k}, e_{k}^{\prime \prime}\right) \\
& P_{1} \rightarrow\left(\rho_{1}, e_{k}^{\prime}\right), \ldots,\left(\rho_{k}, e_{k}^{\prime \prime}\right), \ldots,\left(\rho_{n}, e_{n}^{\prime}\right)
\end{aligned}
$$

Subsubsubcase $e_{k}^{\prime}$ reduces: Again a simple application of process and program reduction rules yields:

\section{3}

32,37 , proc. red.

$3,4,38$, prog. red.

$$
\begin{aligned}
& \left(\rho_{k}, e_{k}^{\prime}\right) \rightarrow^{n, k}\left(\rho_{k}^{\prime}, e_{k}^{\prime \prime \prime}\right) \\
& \left(\rho_{k},\left(e_{k}^{\prime} ; e_{k}^{\prime \prime}\right)\right) \rightarrow^{n, k}\left(\rho_{k}^{\prime},\left(e_{k}^{\prime \prime \prime}, e_{k}^{\prime \prime}\right)\right) \\
& P_{1} \rightarrow\left(\rho_{1}, e_{k}^{\prime}\right), \ldots,\left(\rho_{k}^{\prime},\left(e_{k}^{\prime \prime \prime}, e_{k}^{\prime \prime}\right)\right), \ldots,\left(\rho_{n}, e_{n}^{\prime}\right)
\end{aligned}
$$

Case the derivation ends with the message rule.

$$
\begin{array}{ll}
\text { rule premise } & \Gamma^{n} \vdash l \neq m \text { true } \\
\text { rule premise } & \Gamma^{n} \vdash D: \text { dtype }
\end{array}
$$

According to inversion for expression formation lemma we have five cases for each of the two $e_{j}, e_{m}$ expressions.

Subcase $e_{l}$ is send $i_{l} i_{l}^{\prime}$ and $e_{m}$ is receive $i_{m} i_{m}^{\prime}$ and $l<m$.

lemma D.31
lemma D.31
lemma D.31
lemma D.31
lemma D.31
lemma D.31
4, lemma D.2
9, lemma D.39
$10, i_{l}$ does not contain references

$$
\begin{aligned}
& \Gamma^{n} \vdash \text { rank }=l \text { true } \\
& \Gamma^{n} \vdash i_{l}=m \text { true } \\
& \Gamma^{n} \vdash i_{l}^{\prime}: D \\
& \Gamma^{n} \vdash i_{m}=l \text { true } \\
& \Gamma^{n} \vdash \text { rank }=m \text { true } \\
& \Gamma^{n} \vdash i_{m}^{\prime}: D \text { ref } \\
& \Gamma^{n, l}, \rho_{l} \vdash i_{l}=m \text { true } \\
& \left(\rho_{l}, i_{l}\right) \downarrow^{n, k}\left(\rho_{l}^{\prime}, m\right): D \\
& i_{l} \downarrow^{n} m
\end{aligned}
$$

By applying to (9-11) similar steps as for $i_{m}$ :

$$
\text { steps 9-11, applied to } i_{m} \quad i_{l} \downarrow^{n} m
$$

We can infer that $\left(\rho_{l}, i_{l}^{\prime}\right) \downarrow^{n, l}\left(\rho_{l}^{\prime}, v\right): D$ and $\left(\rho_{m}, i_{m}^{\prime}\right) \downarrow^{n, m}\left(\rho_{m}^{\prime}, r\right): D$ via weakening and that evaluation always succeeds (lemma D.39):

$$
\text { 5, lemma D.2 } \quad \Gamma^{n, l}, \rho_{l} \vdash i_{l}^{\prime}: D
$$




$$
\begin{array}{ll}
\text { 13, lemma D.39 } & \left(\rho_{l}, i_{l}^{\prime}\right) \downarrow^{n, l}\left(\rho_{l}^{\prime}, v\right): D \\
\text { 8, lemma D.2 } & \Gamma^{n, m}, \rho_{m} \vdash i_{m}^{\prime}: D \text { ref } \\
\text { 15, lemma D.39 } & \left(\rho_{m}, i_{m}^{\prime}\right) \downarrow^{n, m}\left(\rho_{m}^{\prime}, r\right): D
\end{array}
$$

Finally, we need to derive $\varepsilon \vdash l \neq m$ true:

$$
\begin{aligned}
& \text { 1, deducibility inversion } \\
& 3 \text {, deducibility and prop inversions } \\
& 7 \text {, deducibility and prop inversions } \\
& \text { 17, lemma D.3, truth formation }
\end{aligned}
$$$$
\Gamma^{n} \vdash l \neq m: \text { prop }
$$$$
\Gamma^{n} \vdash l: \text { int }
$$$$
\Gamma^{n} \vdash m: \text { int }
$$

And putting all together:

$$
\begin{gathered}
q_{1}, \ldots,\left(\rho_{l}, \text { send } i_{l} i_{l}^{\prime}\right), \ldots, q_{m-1},\left(\rho_{m}, \text { receive } i_{m} i_{m}^{\prime}\right), \ldots, q_{n} \rightarrow \\
q_{1}, \ldots,\left(\rho_{l}^{\prime}, \text { skip }\right), \ldots, q_{m-1},\left(\rho_{m}^{\prime}[r:=v], \text { skip }\right), \ldots, q_{n}
\end{gathered}
$$

Subcase $e_{l}$ is send $i_{l} i_{l}^{\prime}$ and $e_{m}$ is receive $i_{m} i_{m}^{\prime}$ and $l>m$ (from (1) we know that the case for $l=m$ does not apply).

Similar to the case above.

Subcase Either $e_{l}$ or $e_{m}$ are not receive or send. We show the case for $e_{l}$, the other is similar.

Subsubcase $e_{l}$ is let $y: D_{1}=i_{1}$ in $e_{l}^{\prime}$

$$
\begin{array}{ll}
\text { lemma D.31 } & \Gamma^{n} \vdash i_{1}: D_{1} \\
\text { lemma D.31 } & y \notin \mathrm{fv}(\text { message } l m D) \\
\text { lemma D.31 } & \Gamma^{n}, y: D_{1} \vdash e_{l}^{\prime}: \text { message } l m D \\
\text { 22, lemma, D.2 } & \Gamma^{n, l}, \rho_{l} \vdash i_{1}: D_{1} \\
\text { 24, lemma D.26, lemma D.30 } & \Gamma^{n, l}, \rho_{l}, y: D_{1} \vdash e_{l}^{\prime}: \text { message } l m D
\end{array}
$$

By applying progress for processes:

$$
\begin{aligned}
& 23,25,26, \text { lemma } 4.3 \\
& 27 \text {, prog. red. }
\end{aligned}
$$

$$
\begin{aligned}
& \left(\rho_{l}, e_{l}\right) \rightarrow{ }^{n, l} q_{l} \\
& \operatorname{skip}_{1}, \ldots,\left(\rho_{l}, e_{l}\right), \operatorname{skip}_{l+1}, \ldots, \operatorname{skip}_{m-1},\left(\rho_{m}, e_{m}\right), \ldots, \operatorname{skip}_{n} \rightarrow \\
& \operatorname{skip}_{1}, \ldots, q_{l}, \operatorname{skip}_{l+1}, \ldots, \operatorname{skip}_{m-1},\left(\rho_{m}, e_{m}\right), \ldots, \operatorname{skip}_{n}
\end{aligned}
$$

Subsubcase $e_{l}$ is if $p$ then $e_{1}$ else $e_{2}$

From inversion of expression formation we get:

$$
\begin{array}{ll}
\text { lemma D.31 } & \Gamma^{n} \vdash p \text { : prop } \\
\text { lemma D.31 } & \Gamma^{n} \vdash e_{1}: \text { message } l m D \\
\text { lemma D.31 } & \Gamma^{n} \vdash e_{2}: \text { message } l m D
\end{array}
$$

We weaken the context with $\rho_{l}$ :

$$
\begin{array}{ll}
\text { 29, lemma D.2 } & \Gamma^{n, l}, \rho_{l} \vdash p: \text { prop } \\
\text { 30, lemma D.26 } & \Gamma^{n, l}, \rho_{l} \vdash e_{1}: \text { message } \operatorname{lm} D \\
\text { 31, lemma D.26 } & \Gamma^{n, l}, \rho_{l} \vdash e_{2}: \text { message } l m D
\end{array}
$$

apply progress for processes:

32, 33, 34, lemma 4.3

35 , prog. red.

$$
\begin{aligned}
& \left(\rho_{l}, \text { if } p \text { then } e_{1} \text { else } e_{2}\right) \rightarrow{ }^{n, l} q_{l} \\
& \operatorname{skip}_{1}, \ldots,\left(\rho_{l}, e_{l}\right), \operatorname{skip}_{l+1}, \ldots, \operatorname{skip}_{m-1},\left(\rho_{m}, e_{m}\right), \ldots, \text { skip }_{n} \rightarrow \\
& \operatorname{skip}_{1}, \ldots, q_{l}, \operatorname{skip}_{l+1}, \ldots, \operatorname{skip}_{m-1},\left(\rho_{m}, e_{m}\right), \ldots, \operatorname{skip}_{n}
\end{aligned}
$$

Subsubcase $e_{l}$ is $e_{1} ; e_{2}$ : 
From inversion of expression formation and weakening we get:

$$
\begin{array}{ll}
\text { lemma D.31 } & \Gamma^{n} \vdash e_{1}: \text { skip } \\
\text { lemma D.31 } & \Gamma^{n} \vdash e_{2}: \text { message } l m D \\
\text { 37, lemma D.26 } & \Gamma^{n, l}, \rho_{l} \vdash e_{1}: \text { skip } \\
\text { 38, lemma D.26 } & \Gamma^{n, l}, \rho_{l} \vdash e_{2} \text { : message } l m D
\end{array}
$$

From progress for processes we have that:

$$
39 \text {, lemma } 4.3 \quad e_{1} \text { is skip or }\left(\rho_{l}, e_{1}\right) \rightarrow^{n, l}\left(\rho_{l}^{\prime}, e_{3}\right)
$$

Subsubsubcase $e_{1}$ is skip: By applying process and program reduction as we in the previous case:

$$
\begin{array}{ll}
40, \text { proc. red. } & \left(\rho_{l}, e_{1} ; e_{2}\right) \rightarrow^{n, l}\left(\rho_{l}, e_{2}\right) \\
42, \text { prog. red. } & \operatorname{skip}_{1}, \ldots,\left(\rho_{l}, e_{l}\right), \ldots, \operatorname{skip}_{m-1},\left(\rho_{m}, e_{m}\right), \ldots, \text { skip }_{n} \rightarrow \\
& \operatorname{skip}_{1}, \ldots,\left(\rho_{l}, e_{2}\right), \ldots, \operatorname{skip}_{m-1},\left(\rho_{m}, e_{m}\right), \ldots, \text { skip }_{n}
\end{array}
$$

Subsubsubcase $\left(\rho_{l}, e_{1}\right) \rightarrow^{n, l}\left(\rho_{l}^{\prime}, e_{3}\right)$ : By applying process and program reductions we get:

40 , proc. red.

$$
\begin{aligned}
& \left(\rho_{l}, e_{1} ; e_{2}\right) \rightarrow{ }^{n, l}\left(\rho_{l}^{\prime}, e_{3} ; e_{2}\right) \\
& \operatorname{skip}_{1}, \ldots,\left(\rho_{l}, e_{l}\right), \ldots, \operatorname{skip}_{m-1},\left(\rho_{m}, e_{m}\right), \ldots, \operatorname{skip}_{n} \rightarrow \\
& \quad \operatorname{skip}_{1}, \ldots,\left(\rho_{l}^{\prime}, e_{3} ; e_{2}\right), \ldots, \operatorname{skip}_{m-1},\left(\rho_{m}, e_{m}\right), \ldots, \operatorname{skip}_{n}
\end{aligned}
$$

